


\title{
The Volume Spectrum of Hyperbolic 4-Manifolds
}

\author{
John G. Ratcliffe and Steven T. Tschantz
}

\section{CONTENTS}

1. Introduction

2. Integral, Congruence Two, Hyperbolic Surfaces

3. Integral, Congruence Two, Hyperbolic 3-Manifolds

4. Integral, Congruence Two, Hyperbolic 4-Manifolds

5. Side-Pairing Coding

6. Tables

Electronic Availability

References

Keywords: hyperbolic manifolds, 4-manifolds, volume, 24-cell AMS Classification: Primary 30F40, 51M10, 53C 25
We construct complete, open, hyperbolic 4-manifolds of smallest volume by gluing together the sides of a regular ideal 24-cell in hyperbolic 4-space. We also show that the volume spectrum of hyperbolic 4-manifolds is the set of all positive integral multiples of $4 \pi^{2} / 3$.

\section{INTRODUCTION}

A hyperbolic manifold is a Riemannian manifold of constant sectional curvature -1 . The set of all volumes of complete hyperbolic $n$-manifolds of finite volume is called the volume spectrum of hyperbolic $n$-manifolds. It has been known for over a hundred years that the volume spectrum of hyperbolic 2manifolds is the set of all positive integral multiples of $2 \pi$. In contrast to dimension two, Jørgensen and Thurston [Thurston 1979] have shown that the volume spectrum of hyperbolic 3-manifolds is a closed, non-discrete, well-ordered subset of the positive real numbers, having order type $\omega^{\omega}$. In particular, there is a smallest positive number that is the volume of a complete hyperbolic 3-manifold. This number is at present unknown.

In this paper, we geometrically construct examples of complete, open, hyperbolic 4-manifolds of smallest volume and show that the volume spectrum of complete, open, hyperbolic 4-manifolds is the set of all positive integral multiples of $4 \pi^{2} / 3$. This implies that the volume spectrum of hyperbolic 4-manifolds is also the set of all positive integral multiples of $4 \pi^{2} / 3$, since the volume of a closed hyperbolic 4 -manifold is also a multiple of $4 \pi^{2} / 3$. All the manifolds constructed in this paper are open, so this paper sheds no light on the volume spectrum of closed hyperbolic 4-manifolds.

The first explicit example of a hyperbolic 4-manifold of finite volume in the literature is the closed hyperbolic 4-manifold constructed by Davis [1985] by gluing together the opposite sides of a regular 120- 
cell in hyperbolic 4-space. Ratcliffe and Tschantz [1994] constructed an explicit example of a complete, open, hyperbolic 4-manifold of finite volume by gluing together the sides of a regular ideal 24-cell in hyperbolic 4-space. The examples of hyperbolic 4-manifolds of smallest volume in this paper are also obtained by gluing together the sides of a regular ideal 24-cell in hyperbolic 4-space.

Hyperbolic 4-manifolds of small volume are currently of interest in cosmology in the theory of quantum gravity. See [Gibbons 1996] where the examples in this paper are considered in the theory of quantum gravity. More examples of hyperbolic 4-manifolds of small volume that are considered in the theory of quantum gravity are given in [Ratcliffe and Tschantz 1998].

We now set up notation in order to describe our examples and further results. A real $(n+1) \times(n+1)$ matrix $A$ is said to be Lorentzian if $A$ preserves the Lorentzian inner product

$$
x \circ y=x_{1} y_{1}+x_{2} y_{2}+\cdots+x_{n} y_{n}-x_{n+1} y_{n+1} .
$$

The hyperboloid model of hyperbolic $n$-space is the metric space

$$
H^{n}=\left\{x \in \mathbb{R}^{n+1}: x \circ x=-1 \text { and } x_{n+1}>0\right\}
$$

with metric $d$ defined by

$$
\cosh d(x, y)=-x \circ y \text {. }
$$

A Lorentzian $(n+1) \times(n+1)$ matrix $A$ is said to be either positive or negative according as $A$ maps $H^{n}$ to $H^{n}$ or $-H^{n}$. The isometries of $H^{n}$ correspond to the positive Lorentzian $(n+1) \times(n+1)$ matrices.

Let $\Gamma^{n}$ be the group of positive Lorentzian $(n+$ $1) \times(n+1)$ matrices with integer entries. The group $\Gamma^{n}$ is an infinite discrete subgroup of the group $O(n, 1)$ of Lorentzian $(n+1) \times(n+1)$ matrices. The principal congruence two subgroup of $\Gamma^{n}$ is the group $\Gamma_{2}^{n}$ of all matrices in $\Gamma^{n}$ that are congruent to the identity matrix modulo two. The congruence two subgroup $\Gamma_{2}^{n}$ is not torsion-free, but it only has 2torsion [Newman 1972, Theorem IX.7].

In this paper, we construct and classify all the hyperbolic space-forms $H^{n} / \Gamma$ where $\Gamma$ is a torsionfree subgroup of minimal index in the congruence two subgroup $\Gamma_{2}^{n}$ for $n=2,3,4$. We call such a space-form an integral, congruence two, hyperbolic $n$-manifold of minimum volume. We show that there are 2, 13, 1171 isometry classes of integral, congruence two, hyperbolic $n$-manifolds of minimum volume for $n=2,3,4$, respectively. These hyperbolic manifolds have smallest volume among all complete hyperbolic $n$-manifolds for $n=2,4$. Thus there are at least 1171 different complete hyperbolic 4-manifolds of smallest volume. By a theorem of Wang [1972], there are only finitely many complete hyperbolic 4-manifolds, up to isometry, with the same finite volume.

The 1171 integral, congruence two, hyperbolic 4manifolds of minimum volume are the simplest complete hyperbolic 4-manifolds of finite volume. They are all constructed by gluing together the sides of a regular ideal 24-cell in hyperbolic 4-space in a particularly simple way. A complete hyperbolic 4-manifold that is obtained by gluing together the sides of a regular ideal 24-cell is called a 24-cell manifold. We shall call an integral, congruence two, hyperbolic 4-manifold of minimum volume a congruence two 24-cell manifold. All but 22 of the 1171 congruence two 24-cell manifolds are nonorientable.

The nonorientable congruence two 24-cell manifolds are far more interesting than the few orientable ones. The first 24-cell manifold we constructed is the nonorientable manifold referred to as the hyperbolic 24-cell space in [Ratcliffe 1994]. It has a symmetry group of order 128 all of whose elements are induced by symmetries of the 24-cell. Of all the congruence two 24-cell manifolds, the hyperbolic 24-cell space is constructed by the most symmetric side-pairing of the 24-cell.

Quite surprisingly, there is a congruence two 24cell manifold with an even larger symmetry group of order 320. This manifold has the largest symmetry group among all the congruence two 24-cell manifolds. If one equates beauty with symmetry, then this manifold is the most beautiful congruence two 24-cell manifold. It has a symmetry of order 5 that cyclically permutes its 5 cusps. This manifold is one of only two congruence two 24-cell manifolds with the property that all of their cusps have the same type. Both of these manifolds are nonorientable.

A nonorientable manifold is double covered by an orientable manifold, and one should think of a nonorientable manifold as an orientable manifold together with an orientation reversing fixed point free involution. In fact, the orientable double covers of 
our nonorientable manifolds are of interest in cosmology [Ratcliffe and Tschantz 1998].

By the Gauss-Bonnet theorem (see [Gromov 1982; Hopf 1926]), the volume of a complete hyperbolic 4manifold $M$ of finite volume is given by

$$
\operatorname{Vol}(M)=\frac{4 \pi^{2}}{3} \chi(M),
$$

where $\chi(M)$ is the Euler characteristic of $M$. We prove that there are complete, open, orientable, hyperbolic 4-manifolds of finite volume whose Euler characteristic is any given positive integer. Therefore the volume spectrum of hyperbolic 4-manifolds is the set of all positive integral multiples of $4 \pi^{2} / 3$.

We also determine the structure of the congruence two subgroup $\Gamma_{2}^{n}$ for $n=2,3,4$. In particular, we show that $\Gamma_{2}^{n}$ is a reflection group with respect to a noncompact right-angled polytope $P^{n}$ in hyperbolic $n$-space for $n=2,3,4$. This implies that $H^{n} / \Gamma_{2}^{n}$ is isometric to $P^{n}$ for $n=2,3,4$. We prove that $\Gamma_{2}^{n}$ has a torsion-free subgroup of finite index $i$ if and only if $i$ is divisible by $2^{n}$ for $n=2,3,4$. We classify, up to isomorphism, all the torsion-free subgroups of $\Gamma_{2}^{n}$ of index $2^{n}$ for $n=2,3,4$. These are the groups whose orbit spaces are the integral, congruence two, hyperbolic $n$-manifolds of minimum volume for $n=2,3,4$. Thus the integral, congruence two, hyperbolic $n$-manifolds of minimum volume are the minimal, nonsingular, covering spaces of the orbifold $P^{n}$ for $n=2,3,4$. The classification of these manifolds in dimension $n$ will play a role in the classification in dimension $n+1$ for $n=2,3$.

This paper is organized as follows: In Sections 2, 3 , and 4 , we determine the structure of the congruence two subgroup $\Gamma_{2}^{n}$ and construct and classify all the congruence two hyperbolic $n$-manifolds of minimum volume for $n=2,3,4$, respectively. In Section 5 , we describe our coding for the side-pairings of a fundamental domain for all these manifolds. In Section 6 , we give tables that list side-pairings and isometry invariants of all 1171 congruence two 24cell manifolds.

\section{INTEGRAL, CONGRUENCE TWO, HYPERBOLIC SURFACES}

In this section, we determine the structure of the congruence two subgroup $\Gamma_{2}^{2}$ of the group $\Gamma^{2}$ of integral, positive, Lorentzian $3 \times 3$ matrices and classify all the congruence two hyperbolic surfaces of minimum area.

According to Fricke $[1891, \S 3]$, the group $\Gamma^{2}$ is a reflection group with respect to a noncompact triangle $\Delta^{2}$ in $H^{2}$ whose Coxeter diagram is

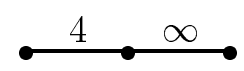

Vertices for $\Delta^{2}$ are $(0,0,1),(\sqrt{2} / 2, \sqrt{2} / 2, \sqrt{2})$, and $(1,0,1)$ (at infinity).

The group $\Gamma^{2}$ is generated by the matrices

$$
\left(\begin{array}{lll}
0 & 1 & 0 \\
1 & 0 & 0 \\
0 & 0 & 1
\end{array}\right),\left(\begin{array}{rrr}
1 & 0 & 0 \\
0 & -1 & 0 \\
0 & 0 & 1
\end{array}\right),\left(\begin{array}{lll}
-1 & -2 & 2 \\
-2 & -1 & 2 \\
-2 & -2 & 3
\end{array}\right),
$$

which represent the reflections in the sides of $\Delta^{2}$. By mapping these matrices into $\mathrm{GL}(3, \mathbb{Z} / 2 \mathbb{Z})$, we see that the index of $\Gamma_{2}^{2}$ in $\Gamma^{2}$ is two.

Let $\Sigma^{2}$ be the group of order two generated by the first matrix in the above list of matrices. Then $\Sigma^{2}$ is a set of coset representatives for $\Gamma_{2}^{2}$ in $\Gamma^{2}$. We therefore have a natural, split, short, exact sequence of groups

$$
1 \rightarrow \Gamma_{2}^{2} \rightarrow \Gamma^{2} \rightarrow \Sigma^{2} \rightarrow 1
$$

We now pass to the conformal disk model $B^{2}$ of the hyperbolic plane. The vertices of $\Delta^{2}$ are now $(0,0),(1-\sqrt{2} / 2,1-\sqrt{2} / 2),(1,0)$. The triangle $\Delta^{2}$ is a triangle of the barycentric subdivision of the ideal square $Q^{2}$ whose vertices are $( \pm 1,0),(0, \pm 1)$. See Figure 1. Let $P^{2}$ be the intersection of $Q^{2}$ with the first quadrant of $\mathbb{R}^{2}$. Then $P^{2}$ is a noncompact right-triangle with vertices $(0,0),(1,0),(0,1)$.

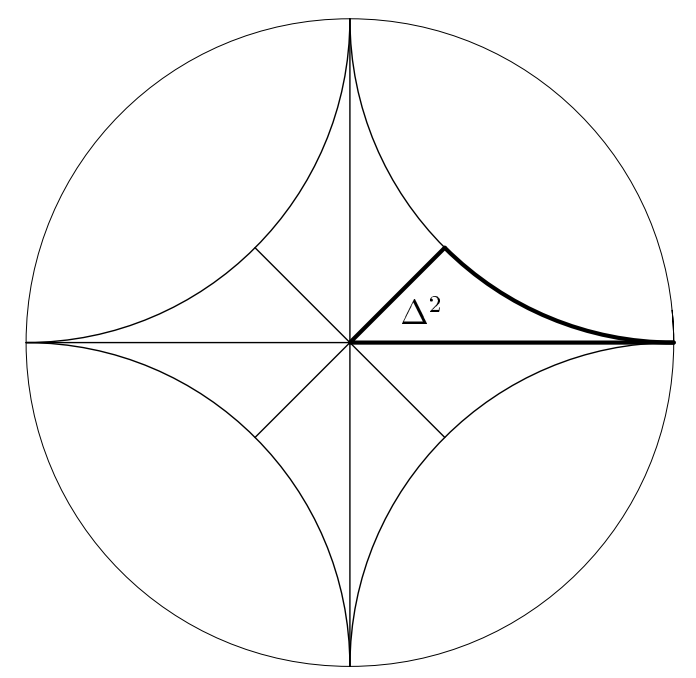

FIGURE 1. The triangle $\Delta^{2}$. 
Observe that $P^{2}=\Sigma^{2} \Delta^{2}$ and $\Sigma^{2}$ is the group of symmetries of $P^{2}$. The Lorentzian matrices that represent the reflections in the sides of $P^{2}$ are

$$
\left(\begin{array}{rrr}
-1 & 0 & 0 \\
0 & 1 & 0 \\
0 & 0 & 1
\end{array}\right),\left(\begin{array}{rrr}
1 & 0 & 0 \\
0 & -1 & 0 \\
0 & 0 & 1
\end{array}\right),\left(\begin{array}{lll}
-1 & -2 & 2 \\
-2 & -1 & 2 \\
-2 & -2 & 3
\end{array}\right) .
$$

These matrices are all in $\Gamma_{2}^{2}$. Now since $\Sigma^{2}$ is a set of coset representatives for $\Gamma_{2}^{2}$ in $\Gamma^{2}$, we have that $P^{2}=\Sigma^{2} \Delta^{2}$ is a fundamental polygon for $\Gamma_{2}^{2}$. We therefore have the following theorem.

Theorem 1. The congruence two subgroup $\Gamma_{2}^{2}$ of the group $\Gamma^{2}$ of integral, positive, Lorentzian $3 \times 3$ matrices is a reflection group with respect to a noncompact triangle $P^{2}$ whose Coxeter diagram is

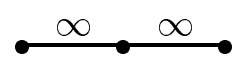

Let $K^{2}$ be the Klein four group generated by the reflections in the vertical and horizontal sides of $P^{2}$. The next corollary follows immediately from Theorem 1.

Corollary 1. Every nonidentity element of $\Gamma_{2}^{2}$ of finite order has order two and every finite subgroup of $\Gamma_{2}^{2}$ is conjugate in $\Gamma_{2}^{2}$ to a subgroup of the Klein four group $K^{2}$.

Let $\Gamma$ be a torsion-free subgroup of $\Gamma_{2}^{2}$ of finite index $i$. Then $M=H^{2} / \Gamma$ is a hyperbolic surface of finite area. By the Gauss-Bonnet theorem, we have

$$
\operatorname{Area}(M)=-2 \pi \chi(M),
$$

where $\chi(M)$ is the Euler characteristic of $M$. Therefore the area of $M$ is a multiple of $2 \pi$. As the area of $P^{2}$ is $\pi / 2$, we deduce that $i$ is divisible by 4 .

Now suppose that $i=4$. Then $K^{2}$ forms a set of coset representatives of $\Gamma$ in $\Gamma_{2}^{2}$ and so the ideal square $Q^{2}=K^{2} P^{2}$ is a fundamental polygon for $\Gamma$. Let $S$ be a side of $Q^{2}$. Then there is a nonidentity element $g$ of $\Gamma$ that pairs a side $S^{\prime}$ of $Q^{2}$ to $S$. Let $k$ be the element of $K^{2}$ that maps $S^{\prime}$ to $S$, and let $r$ be the reflection in the side $S$. Then $g k r$ leaves $S$ invariant. The only elements of $\Gamma_{2}^{2}$ that leave $S$ invariant are the identity and $r$. We cannot have $g k r=r$, since $g$ has infinite order. Therefore $g k r=$ 1. Thus the side-pairing transformations of $Q^{2}$ are of the form $r k$ where $k$ is in $K^{2}$ and $r$ is the reflection in a side of $Q^{2}$.
Now $\Gamma$ is generated by the side-pairing transformations of $Q^{2}$, and so $\Gamma$ is determined by the sidepairing of $Q^{2}$. There are only three side-pairings for $Q^{2}$ of the above form. Two of these pairings are equivalent by a symmetry of $Q^{2}$ and yield the hyperbolic thrice-punctured sphere $M_{1}^{2}$ and the third pairing yields the symmetric, hyperbolic, twice-punctured, projective plane $M_{2}^{2}$. See Figure 2. Thus we have the following theorem.

Theorem 2. There are, up to isometry, exactly two hyperbolic space-forms $H^{2} / \Gamma$ where $\Gamma$ is a torsionfree subgroup of minimal index in the congruence two subgroup $\Gamma_{2}^{2}$ of the group $\Gamma^{2}$ of integral, positive, Lorentzian $3 \times 3$ matrices.

Theorem 3. The congruence two group $\Gamma_{2}^{2}$ has a torsion-free subgroup of index $i$ if and only if $i$ is divisible by 4.

Proof. We have already shown that every torsion-free subgroup of $\Gamma_{2}^{2}$ has index divisible by 4 . Let $\Gamma$ be a torsion-free subgroup of $\Gamma_{2}^{2}$ of index four. Then $\Gamma$ is a free group of rank two. Therefore $\Gamma$ maps homomorphically onto $\mathbb{Z}$. Hence $\Gamma$ has a subgroup of index $i$ for each positive integer $i$. Therefore $\Gamma_{2}^{2}$ has a torsion-free subgroup of index $4 j$ for each positive integer $j$.

\section{INTEGRAL, CONGRUENCE TWO, HYPERBOLIC 3-MANIFOLDS}

In this section, we determine the structure of the congruence two subgroup $\Gamma_{2}^{3}$ of the group $\Gamma^{3}$ of integral, positive, Lorentzian $4 \times 4$ matrices and classify all integral, congruence two, hyperbolic 3-manifolds of minimum volume. Coxeter [1950] proved that the group $\Gamma^{3}$ is a reflection group with respect to a noncompact tetrahedron $\Delta^{3}$ in $H^{3}$ (see Figure 3 ) whose Coxeter diagram is

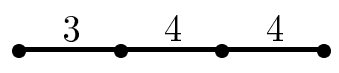

Vertices for $\Delta^{3}$ are

$$
\begin{gathered}
(0,0,0,1),(\sqrt{6} / 6, \sqrt{6} / 6, \sqrt{6} / 6, \sqrt{6} / 2) \\
(\sqrt{2} / 2, \sqrt{2} / 2,0, \sqrt{2}), \text { and }(1,0,0,1) \text { (at infinity). }
\end{gathered}
$$



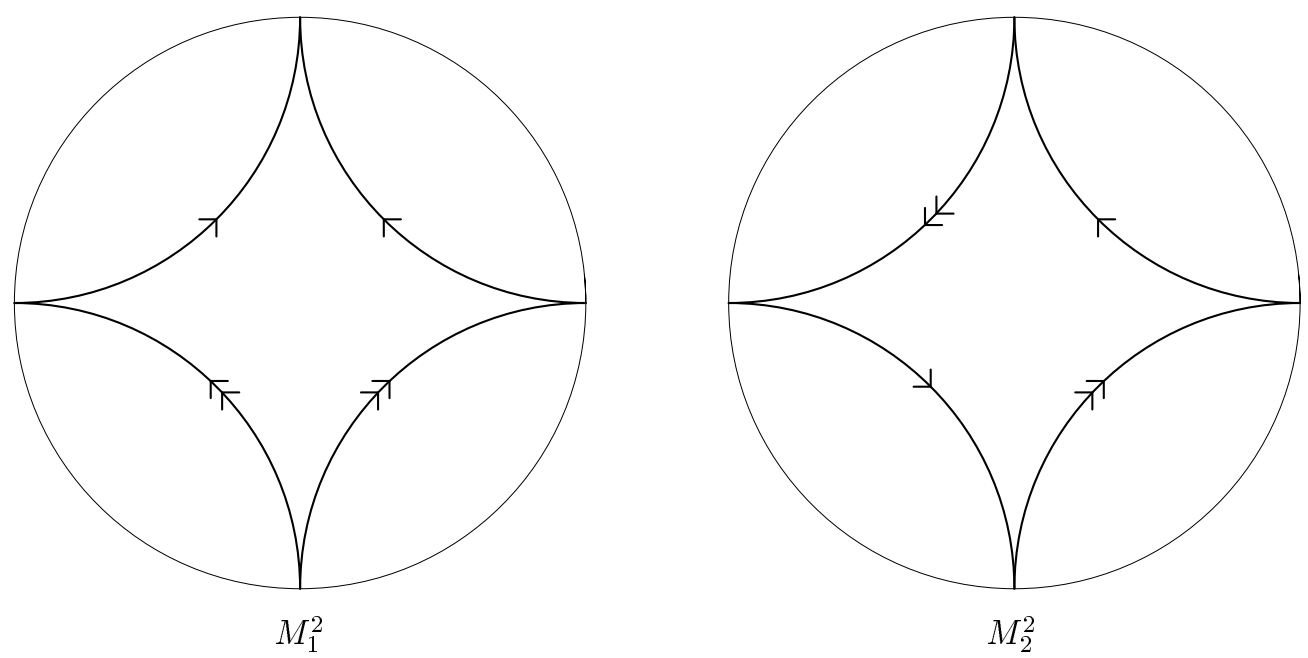

FIGURE 2. The 3-punctured sphere and the 2-punctured projective plane.

The group $\Gamma^{3}$ is generated by the four matrices

$$
\begin{gathered}
\left(\begin{array}{rrrr}
0 & 1 & 0 & 0 \\
1 & 0 & 0 & 0 \\
0 & 0 & 1 & 0 \\
0 & 0 & 0 & 1
\end{array}\right), \quad\left(\begin{array}{llll}
1 & 0 & 0 & 0 \\
0 & 0 & 1 & 0 \\
0 & 1 & 0 & 0 \\
0 & 0 & 0 & 1
\end{array}\right), \\
\left(\begin{array}{rrrr}
0 & -1 & -1 & 1 \\
-1 & 0 & -1 & 1 \\
-1 & -1 & 0 & 1 \\
-1 & -1 & -1 & 2
\end{array}\right), \quad\left(\begin{array}{rrrr}
1 & 0 & 0 & 0 \\
0 & 1 & 0 & 0 \\
0 & 0 & -1 & 0 \\
0 & 0 & 0 & 1
\end{array}\right),
\end{gathered}
$$

which represent the reflections in the sides of $\Delta^{3}$. By mapping these matrices into $\mathrm{GL}(4, \mathbb{Z} / 2 \mathbb{Z})$ and computing the order of the group that their images generate, we deduce that the index of $\Gamma_{2}^{3}$ in $\Gamma^{3}$ is 12 .

Let $\Sigma^{3}$ be the group generated by the first three matrices in the above list of matrices. These are the generators of $\Gamma^{3}$ that project to nonzero elements of $\mathrm{GL}(4, \mathbb{Z} / 2 \mathbb{Z})$. Then $\Sigma^{3}$ is the group generated by the reflections in the three sides of $\Delta^{3}$ incident with the vertex $(\sqrt{6} / 6, \sqrt{6} / 6, \sqrt{6} / 6, \sqrt{6} / 2)$. Therefore $\Sigma^{3}$ is isomorphic to a spherical triangle reflection group whose Coxeter diagram is obtained from the Coxeter diagram of $\Gamma_{2}^{3}$ by deleting its third vertex and its adjoining edges. Hence, the Coxeter diagram of $\Sigma^{3}$ is the disjoint union of an edge labeled by 3 and a vertex. Therefore $\Sigma^{3}$ is the direct product of the dihedral group $D^{3}$ of order six generated by the first two matrices and the group of order two generated by the third matrix in the above list of matrices. Thus $\Sigma^{3}$ has order 12 .
Now $\Sigma^{3}$ injects into $\mathrm{GL}(4, \mathbb{Z} / 2 \mathbb{Z})$, since it has the same order as its image. Hence $\Sigma^{3}$ is a set of coset representatives for $\Gamma_{2}^{3}$ in $\Gamma^{3}$. We therefore have a natural, split, short, exact sequence of groups

$$
1 \rightarrow \Gamma_{2}^{3} \rightarrow \Gamma^{3} \rightarrow \Sigma^{3} \rightarrow 1 .
$$

We now pass to the conformal ball model $B^{3}$ of hyperbolic 3 -space. The vertices of $\Delta^{3}$ are now

$$
\begin{gathered}
(0,0,0), \quad(1-\sqrt{6} / 3,1-\sqrt{6} / 3,1-\sqrt{6} / 3), \\
(1-\sqrt{2} / 2,1-\sqrt{2} / 2,0), \quad(1,0,0),
\end{gathered}
$$

and $\Delta^{3}$ is a tetrahedron of the barycentric subdivision of the ideal octahedron $O^{3}$ whose vertices are $( \pm 1,0,0),(0, \pm 1,0),(0,0, \pm 1)$.

Let $T^{3}$ be the intersection of $O^{3}$ with the positive octant of $\mathbb{R}^{3}$. Then $T^{3}$ is a noncompact tetrahedron with vertices $(0,0,0),(1,0,0),(0,1,0),(0,0,1)$. The dihedral angles of $T^{3}$ along the edges joining the ideal vertices of $T^{3}$ are all $\pi / 4$. The barycentric subdivision of $O^{3}$ subdivides $T^{3}$ into six copies of $\Delta^{3}$ that are the images of $\Delta^{3}$ under the elements of the dihedral group $D^{3}$. See Figure 3 .

Let $P^{3}$ be the union of $T^{3}$ and the tetrahedron $\left(T^{3}\right)^{\prime}$ obtained by reflecting $T^{3}$ in the side of $T^{3}$ spanned by its ideal vertices, that is, the front face of $T^{3}$ in Figure 3. Then $P^{3}$ is a noncompact polyhedron with six sides and five vertices, two actual, $(0,0,0)$ and $(1 / 3,1 / 3,1 / 3)$, and three ideal. See Figure 4 . The dihedral angles of $P^{3}$ are all $\pi / 2$. Observe that $P^{3}=\Sigma^{3} \Delta^{3}$ and $\Sigma^{3}$ is the group of symmetries of $P^{3}$. 


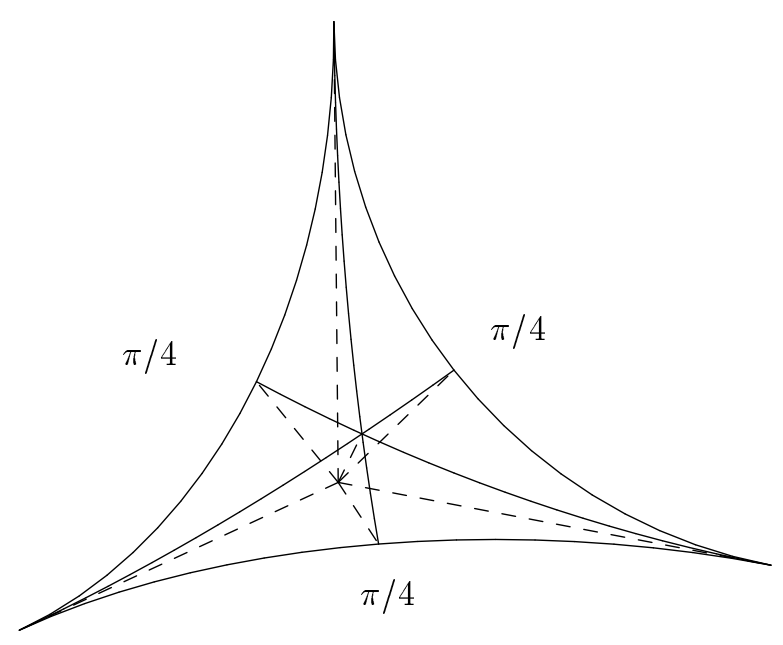

FIGURE 3. The subdivision of $T^{3}$ into six copies of $\Delta^{3}$.

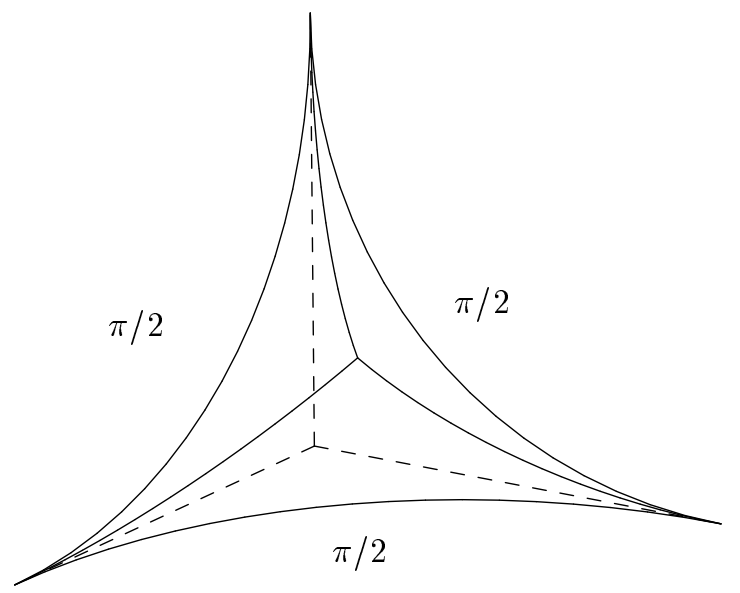

FIGURE 4. The polyhedron $P^{3}$.

The Lorentzian matrices that represent the reflections in the sides of $P^{3}$ are

$$
\begin{aligned}
& \left(\begin{array}{rrrr}
-1 & 0 & 0 & 0 \\
0 & 1 & 0 & 0 \\
0 & 0 & 1 & 0 \\
0 & 0 & 0 & 1
\end{array}\right), \quad\left(\begin{array}{rrrr}
1 & 0 & 0 & 0 \\
0 & -1 & 0 & 0 \\
0 & 0 & 1 & 0 \\
0 & 0 & 0 & 1
\end{array}\right), \\
& \left(\begin{array}{rrrr}
1 & 0 & 0 & 0 \\
0 & 1 & 0 & 0 \\
0 & 0 & -1 & 0 \\
0 & 0 & 0 & 1
\end{array}\right), \quad\left(\begin{array}{rrrr}
1 & 0 & 0 & 0 \\
0 & -1 & -2 & 2 \\
0 & -2 & -1 & 2 \\
0 & -2 & -2 & 3
\end{array}\right), \\
& \left(\begin{array}{rrrr}
-1 & 0 & -2 & 2 \\
0 & 1 & 0 & 0 \\
-2 & 0 & -1 & 2 \\
-2 & 0 & -2 & 3
\end{array}\right), \quad\left(\begin{array}{rrrr}
-1 & -2 & 0 & 2 \\
-2 & -1 & 0 & 2 \\
0 & 0 & 1 & 0 \\
-2 & -2 & 0 & 3
\end{array}\right)
\end{aligned}
$$

All are in $\Gamma_{2}^{3}$. Since $\Sigma^{3}$ is a set of coset representatives for $\Gamma_{2}^{3}$ in $\Gamma^{3}$, we see that $P^{3}=\Sigma^{3} \Delta^{3}$ is a fundamental polyhedron for $\Gamma_{2}^{3}$. Therefore:

Theorem 4. The congruence two subgroup $\Gamma_{2}^{3}$ of the group $\Gamma^{3}$ of integral, positive, Lorentzian $4 \times 4 \mathrm{ma}^{-}$ trices is a reflection group with respect to the noncompact polyhedron $P^{3}$.

Let $K^{3}$ be the elementary 2-group of order 8 generated by the three reflections in the coordinate planes of $\mathbb{R}^{3}$. We shall identify $K^{3}$ with the corresponding subgroup of $\Gamma^{3}$ generated by the first three matrices in last displayed list of matrices. The next corollary follows immediately from Theorem 4 .

Corollary 2. Every nonidentity element of $\Gamma_{2}^{3}$ of finite order has order two, every finite subgroup of $\Gamma_{2}^{3}$ is conjugate in $\Gamma^{3}$ to a subgroup of the elementary 2 group $K^{3}$, and there are two conjugacy classes of maximal finite subgroups of $\Gamma_{2}^{3}$ in $\Gamma_{2}^{3}$ corresponding to the two actual vertices of $P^{3}$.

Let $\Gamma$ be a torsion-free subgroup of $\Gamma_{2}^{3}$ of finite index. Then $K^{3}$ acts freely on the set of cosets of $\Gamma$ in $\Gamma_{2}^{3}$ by $g \Gamma \mapsto k g \Gamma$, since $\Gamma$ is torsion-free. Therefore $\left|K^{3}\right|=8$ divides $\left[\Gamma_{2}^{3}: \Gamma\right]$. Now suppose that $\left[\Gamma_{2}^{3}\right.$ : $\Gamma]=8$. Then the set $Q^{3}=K^{3} P^{3}$ is a fundamental polyhedron for $\Gamma$ (Figure 5). It is a rhombic dodecahedron with 14 vertices, 8 actual $\left( \pm \frac{1}{3}, \pm \frac{1}{3}, \pm \frac{1}{3}\right)$, and 6 ideal $( \pm 1,0,0),(0, \pm 1,0),(0,0, \pm 1)$. It has the same group of symmetries as the cube with vertices $\left( \pm \frac{1}{3}, \pm \frac{1}{3}, \pm \frac{1}{3}\right)$. All its dihedral angles are $\pi / 2$.

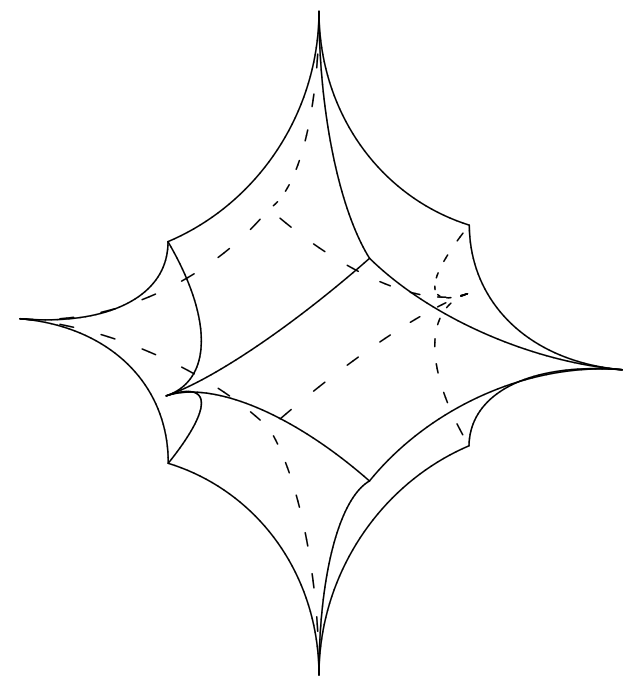

FIGURE 5. The polyhedron $Q^{3}$. 
Let $S$ be a side of $Q^{3}$. Then there is a nonidentity element $g$ of $\Gamma$ that pairs a side $S^{\prime}$ of $Q^{3}$ to $S$. Let $k$ be the element of $K^{3}$ that maps $S^{\prime}$ to $S$, and let $r$ be the reflection in the side $S$. Then $g k r$ leaves $S$ invariant. The only elements of $\Gamma_{2}^{3}$ that leave $S$ invariant are the identity, the reflection $r$, the reflection $s$ in the coordinate plane $P$ perpendicular to $S$, and the $180^{\circ}$ rotation $r s=s r$ about the line $P \cap S$. Note that $s$ is in $K^{3}$. We cannot have $g k r=r$ or $g k r=s r$, since $g$ has infinite order. Therefore $g k r=1$ or $g k r=s$. Hence, the side-pairing transformations of $Q^{3}$ are of the form $r k$ where $k$ is in $K^{3}$ and $r$ is the reflection in a side of $Q^{3}$.

Now $\Gamma$ is generated by the side-pairing transformations for $Q^{3}$, and so $\Gamma$ is determined by the sidepairing of $Q^{3}$. The side-pairing for $Q^{3}$ restricts to a side-pairing for each of the three copies of $Q^{2}$ obtained from $Q^{3}$ by intersecting $Q^{3}$ with a coordinate plane. Extending the 3 possible side-pairings for $Q^{2}$ that yield an integral, congruence two, hyperbolic surface on each of the 3 copies of $Q^{2}$ in $Q^{3}$ yields 1728 side-pairings for $Q^{3}$ of the above form. Only 107 of these satisfy the conditions of Poincaré's fundamental polyhedron theorem [Ratcliffe 1994] for the gluing of a complete hyperbolic 3-manifold. These 107 side-pairings for $Q^{3}$ fall into 20 equivalence classes under equivalence by a symmetry of $Q^{3}$. The classification of the hyperbolic 3 -manifolds that correspond to these side-pairings of $Q^{3}$ is summarized in our next theorem.

Theorem 5. There are, up to isometry, exactly 13 hyperbolic space-forms $H^{3} / \Gamma$ where $\Gamma$ is a torsionfree subgroup of minimal index in the congruence two subgroup $\Gamma_{2}^{3}$ of the group $\Gamma^{3}$ of integral, positive, Lorentzian $4 \times 4$ matrices. Only three of these manifolds are orientable.

Proof. All the 107 side-pairings for $Q^{3}$ identify the 8 vertices $( \pm 1 / 3, \pm 1 / 3, \pm 1 / 3)$ of $Q^{3}$ to one point.
Notice that each of the vertices $( \pm 1 / 3, \pm 1 / 3, \pm 1 / 3)$ is the corner vertex of a right-angled corner (like the corner of a room). This suggests a cut and paste operation on $Q^{3}$. Cut $Q^{3}$ along the three coordinate planes into 8 copies of the polyhedron $P^{3}$, turn around each of the 8 copies of $P^{3}$, and reassemble the polyhedron $Q^{3}$ according to the gluing pattern of the side-pairing of $Q^{3}$ so that the vertices $( \pm 1 / 3, \pm 1 / 3, \pm 1 / 3)$ are all glued together at $(0,0,0)$. We call this an inside-out operation on $Q^{3}$. Each of the 107 side-pairings of $Q^{3}$ induces a new side-pairing on $Q^{3}$ after an inside-out operation on $Q^{3}$ that yields the same manifold. After comparing the new side-pairings with the old ones, up to symmetry of $Q^{3}$, the number of manifolds is reduced to 13. Table 1 lists side-pairings and isometric invariants for the 13 manifolds.

We denote the manifolds in Table 1 by $M_{1}^{3}, \ldots$, $M_{13}^{3}$ indexed by the row number in the column that says $N$. The column headed by $S P$ describes the side-pairing of $Q^{3}$ in a coded form that will be explained in Section 5. The column headed by $O$ indicates the orientability of the manifolds with 1 for orientable and 0 for nonorientable. A manifold in Table 1 is orientable if and only if all the side-pairing transformations of the corresponding side-pairing of $Q^{3}$ are orientation preserving.

The column of Table 1 headed by $C$ lists the number of cusps of the manifolds. The link of each cusp is either a torus or a Klein bottle. The column headed by $L T$ indicates the link type of each cusp with $\mathrm{T}$ representing a torus and $\mathrm{K}$ a Klein bottle. The column headed by $S$ lists the number of symmetries of the manifold. The column headed by $H_{1}$ lists the first homology groups of the manifolds with the 3 digit number $a b c$ representing $\mathbb{Z}^{a} \oplus \mathbb{Z}_{2}^{b} \oplus \mathbb{Z}_{4}^{c}$. The column headed by $H_{2}$ lists the second homology groups of the manifolds with the entry a representing $\mathbb{Z}^{a}$. Notice that the 4-cusped manifolds are classified by their first homology groups.

$\begin{array}{rrrrrrrc}N & S P & O & C & S & H_{1} & H_{2} & L T \\ 1 & 142 & 1 & 3 & 48 & 300 & 2 & \text { TTT } \\ 2 & 147 & 1 & 3 & 16 & 300 & 2 & \text { TTT } \\ 3 & 143 & 0 & 3 & 8 & 300 & 2 & \text { KTT } \\ 4 & 156 & 0 & 3 & 8 & 300 & 2 & \text { KTT }\end{array}$

$\begin{array}{rcccrccc}N & S P & O & C & S & H_{1} & H_{2} & L T \\ 5 & 357 & 0 & 3 & 16 & 220 & 1 & \mathrm{KKT} \\ 6 & 136 & 0 & 3 & 8 & 220 & 1 & \mathrm{KKT} \\ 7 & 153 & 0 & 3 & 16 & 201 & 1 & \mathrm{KKT} \\ 8 & 157 & 0 & 3 & 8 & 201 & 1 & \mathrm{KKT} \\ 9 & 367 & 0 & 3 & 8 & 102 & 0 & \mathrm{KKK}\end{array}$

TABLE 1. Minimal volume, integral, congruence 2, hyperbolic 3-manifolds. 
The side-pairings of $Q^{3}$ for the 3-cusped manifolds have the property that opposite ideal vertices of $Q^{3}$ are identified. Consequently, each boundary of a maximum open cusp of a 3-cusped manifold is tangent to itself in the two points represented by $(0,0,0)$ and $( \pm 1 / 3, \pm 1 / 3, \pm 1 / 3)$. Therefore these two points are canonical points of the manifold.

The ball centered at $(0,0,0)$ inscribed in $Q^{3}$ meets the boundary of $Q^{3}$ in the centers of the 12 sides of $Q^{3}$. Let $M$ be one of the 3-cusped manifolds and let $c$ be the point of $M$ represented by $(0,0,0)$. Then the boundary of the maximum open ball centered at $c$ in $M$ is tangent to itself in the six point $c_{1}, \ldots, c_{6}$ represented by the centers of the sides of $Q^{3}$.

Let $\varphi: M \rightarrow M^{\prime}$ be an isometry from $M$ to the 3-cusped manifold $M^{\prime}$. Let $c^{\prime}$ be the point of $M^{\prime}$ represented by $(0,0,0)$. By applying an inside-out operation to $Q^{3}$ if $\varphi(c) \neq c^{\prime}$, we may assume that $\varphi(c)=c^{\prime}$. Let $c_{1}^{\prime}, \ldots, c_{6}^{\prime}$ be the six points of $M^{\prime}$ represented by the centers of the sides of $Q^{3}$. Then $\varphi$ must map the set $\left\{c_{1}, \ldots, c_{6}\right\}$ to the set $\left\{c_{1}^{\prime}, \ldots, c_{6}^{\prime}\right\}$. Consequently $\varphi$ lifts to a symmetry of $Q^{3}$. Thus, an isometry between two 3 -cusped manifolds is induced either by a symmetry of $Q^{3}$ or by an inside-out operation on $Q^{3}$ followed by a symmetry of $Q^{3}$. As the 13 manifolds are already classified up to such an isometry, the classification is complete.

We next show that the three orientable manifolds $M_{1}^{3}, M_{2}^{3}, M_{10}^{3}$ are homeomorphic to complements of links in the 3 -sphere. When we pass to the upper half space model $U^{3}$ of hyperbolic 3 -space, the vertices of $Q^{3}$ become $(0,0,0),( \pm 1,0,0),(0, \pm 1,0)$, $( \pm 1 / 3, \pm 1 / 3,1 / 3),( \pm 1, \pm 1,1)$, and $\infty$. We identify the boundary plane of $U^{3}$ with the complex plane $\mathbb{C}$. Then the orientation preserving isometries of $U^{3}$ correspond to the elements of the group $\operatorname{PSL}(2, \mathbb{C})$ and the side-pairing transformations for the manifolds $M_{1}^{3}, M_{2}^{3}, M_{10}^{3}$ in Table 1 correspond to elements of the Picard group $\operatorname{PSL}(2, \mathbb{Z}[i])$. Consequently, the manifolds $M_{1}^{3}, M_{2}^{3}, M_{10}^{3}$ correspond to torsion-free subgroups of the Picard group.

The hyperbolic manifolds $M_{1}^{3}, \ldots, M_{13}^{3}$ all have the same volume as $Q^{3}$. The polyhedron $Q^{3}$ is built up from 96 copies of the tetrahedron $\Delta^{3}$, so its volume equals $96 \operatorname{Vol}\left(\Delta^{3}\right)$. According to Bianchi $[1892$, $\S 12]$, the Picard group has a natural extension, by the Klein four group $\{ \pm 1, \pm i\}$, that is isomorphic to $\Gamma^{3}$. Therefore, we have

$$
\operatorname{Vol}\left(U^{3} / \operatorname{PSL}(2, \mathbb{Z}[i])\right)=4 \operatorname{Vol}\left(\Delta^{3}\right) .
$$

Hence, we have

$$
\operatorname{Vol}\left(Q^{3}\right)=24 \operatorname{Vol}\left(U^{3} / \operatorname{PSL}(2, \mathbb{Z}[i])\right)
$$

Therefore, the manifolds $M_{1}^{3}, M_{2}^{3}, M_{10}^{3}$ correspond to torsion-free subgroups of the Picard group of index 24. Milnor [1982] has shown that

$$
\operatorname{Vol}\left(U^{3} / \operatorname{PSL}(2, \mathbb{Z}[i])\right)=\frac{2}{3} \mathcal{L}(\pi / 4),
$$

where $\mathcal{L}$ is the Lobachevsky function [Milnor 1982]. Therefore, we have

$$
\operatorname{Vol}\left(Q^{3}\right)=16 \mathcal{L}(\pi / 4)=7.3277247 \ldots
$$

From the classification of all the index 24 torsionfree subgroups of the Picard group given by Brunner, Frame, Lee, and Wielenberg [Brunner et al. 1984], we deduce that the 4-cusped manifold $M_{10}^{3}$ is homeomorphic to the link complement $8_{2}^{4}$. One can also derive a presentation for the fundamental group of $M_{10}^{3}$ from the side-pairing for $M_{10}^{3}$ in Table 1 and transform the presentation into the presentation for the group of the link complement $8_{2}^{4}$ given by Wielenberg [1978]. It then follows from Mostow's rigidity theorem that $M_{10}^{3}$ is homeomorphic to the link complement $8_{2}^{4}$.

Cutting off the top half $\left(T^{3}\right)^{\prime}$ of each of the 8 copies of $P^{3}$ in $Q^{3}$ leaves a regular ideal octahedron. The eight copies of the tetrahedron $\left(T^{3}\right)^{\prime}$ can be assembled around their corner points to form a regular ideal octahedron. Therefore each of the manifolds $M_{1}^{3}, \ldots, M_{13}^{3}$ can be obtained by gluing together two regular ideal octahedrons along their sides. The manifold $M_{2}^{3}$ has an inside-out symmetry that interchanges the two octahedrons and has no fixed points. The quotient space under the action of this inside-out symmetry of $M_{2}^{3}$ is the Whitehead link complement obtained by gluing together the sides of a regular octahedron as in [Thurston 1997]. From the classification of all the index 24 torsion-free subgroups of the Picard group [Brunner et al. 1984], we deduce that $M_{2}^{3}$ is homeomorphic to the link complement $8_{9}^{3}$. One can also derive a presentation for the fundamental group of $M_{2}^{3}$ from the side-pairing for $M_{2}^{3}$ in Table 1 and transform the presentation into the presentation for the group of the link complement $8_{9}^{3}$ given by Wielenberg [1978]. 
It then follows from Mostow's rigidity theorem that $M_{2}^{3}$ is homeomorphic to the link complement $8_{9}^{3}$.

The manifold $M_{1}^{3}$ is homeomorphic to the link complement $6_{2}^{3}$ (Borromean rings), since the corresponding side-pairing of two regular ideal octahedrons is the one given by Thurston [1997]. The sidepairing of $Q^{3}$ in Table 1 for the Borromean rings has also been described by Hilden, Lozano, and Montesinos [Hilden et al. 1992].

Theorem 6. The congruence two group $\Gamma_{2}^{3}$ has a torsion-free subgroup of index $i$ if and only if $i$ is divisible by 8.

Proof. We have already shown that every torsionfree subgroup of $\Gamma_{2}^{3}$ has index divisible by 8 . From Table 2 we see that $\Gamma_{2}^{3}$ has a torsion-free subgroup $\Gamma$ whose first homology group has an infinite cyclic summand. Therefore $\Gamma$ maps homomorphically onto $\mathbb{Z}$. Hence $\Gamma$ has a subgroup of index $i$ for each positive integer $i$. Therefore $\Gamma_{2}^{3}$ has a torsion-free subgroup of index $8 j$ for each positive integer $j$.

\section{INTEGRAL, CONGRUENCE TWO, HYPERBOLIC 4-MANIFOLDS}

In this section, we determine the structure of the congruence two subgroup $\Gamma_{2}^{4}$ of the group $\Gamma^{4}$ of integral, positive, Lorentzian $5 \times 5$ matrices and classify all the integral, congruence two, hyperbolic 4manifolds of minimum volume. Vinberg [1967] has proved that the group $\Gamma^{4}$ is a reflection group with respect to a noncompact 4 -simplex $\Delta^{4}$ in $H^{4}$ whose Coxeter diagram is

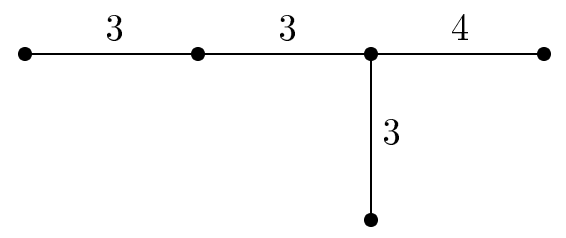

Vertices for $\Delta^{4}$ are

$$
\begin{aligned}
& (0,0,0,0,1), \\
& (\sqrt{6} / 6, \sqrt{6} / 6, \sqrt{6} / 6,0, \sqrt{6} / 2), \\
& (\sqrt{2} / 2, \sqrt{2} / 2,0,0, \sqrt{2}), \\
& (\sqrt{5} / 5, \sqrt{5} / 5, \sqrt{5} / 5, \sqrt{5} / 5,3 \sqrt{5} / 5), \\
& (1,0,0,0,1) \quad \text { (at infinity). }
\end{aligned}
$$

The group $\Gamma^{4}$ is generated by the five matrices

$$
\begin{aligned}
& \left(\begin{array}{lllll}
0 & 1 & 0 & 0 & 0 \\
1 & 0 & 0 & 0 & 0 \\
0 & 0 & 1 & 0 & 0 \\
0 & 0 & 0 & 1 & 0 \\
0 & 0 & 0 & 0 & 1
\end{array}\right), \quad\left(\begin{array}{lllll}
1 & 0 & 0 & 0 & 0 \\
0 & 0 & 1 & 0 & 0 \\
0 & 1 & 0 & 0 & 0 \\
0 & 0 & 0 & 1 & 0 \\
0 & 0 & 0 & 0 & 1
\end{array}\right), \\
& \left(\begin{array}{lllll}
1 & 0 & 0 & 0 & 0 \\
0 & 1 & 0 & 0 & 0 \\
0 & 0 & 0 & 1 & 0 \\
0 & 0 & 1 & 0 & 0 \\
0 & 0 & 0 & 0 & 1
\end{array}\right) \\
& \left(\begin{array}{rrrrr}
0 & -1 & -1 & 0 & 1 \\
-1 & 0 & -1 & 0 & 1 \\
-1 & -1 & 0 & 0 & 1 \\
0 & 0 & 0 & 1 & 0 \\
-1 & -1 & -1 & 0 & 2
\end{array}\right), \quad\left(\begin{array}{rrrrr}
1 & 0 & 0 & 0 & 0 \\
0 & 1 & 0 & 0 & 0 \\
0 & 0 & 1 & 0 & 0 \\
0 & 0 & 0 & -1 & 0 \\
0 & 0 & 0 & 0 & 1
\end{array}\right)
\end{aligned}
$$

which represent the reflections in the sides of $\Delta^{4}$. By mapping these matrices into $\mathrm{GL}(5, \mathbb{Z} / 2 \mathbb{Z})$ and computing the order of the group that their images generate, we deduce that the index of $\Gamma_{2}^{4}$ in $\Gamma^{4}$ is 120.

Let $\Sigma^{4}$ be the group generated the first four matrices in the above list of matrices. These are the generators of $\Gamma^{4}$ that project to nonzero elements of $\operatorname{GL}(5, \mathbb{Z} / 2 \mathbb{Z})$. Then $\Sigma^{4}$ is the group generated by the reflections in the sides of $\Delta^{4}$ incident with the vertex $(\sqrt{5} / 5, \sqrt{5} / 5, \sqrt{5} / 5, \sqrt{5} / 5,3 \sqrt{5} / 5)$. Thus $\Sigma^{4}$ is isomorphic to a spherical tetrahedral reflection group whose Coxeter diagram is obtained from the Coxeter diagram of $\Gamma_{2}^{4}$ by deleting its right most vertex and its adjoining edge. Hence $\Sigma^{4}$ has the Coxeter diagram

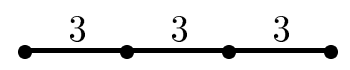

Therefore $\Sigma^{4}$ is isomorphic to the group of symmetries of a regular 4-simplex and so $\Sigma^{4}$ is isomorphic to the symmetric group $S_{5}$. Thus $\Sigma^{4}$ has order 120 .

Now $\Sigma^{4}$ injects into $G L(5, \mathbb{Z} / 2 \mathbb{Z})$, since it has the same order as its image. Hence $\Sigma^{4}$ is a set of coset representatives for $\Gamma_{2}^{4}$ in $\Gamma^{4}$. We therefore have a natural, split, short, exact sequence of groups

$$
1 \rightarrow \Gamma_{2}^{4} \rightarrow \Gamma^{4} \rightarrow \Sigma^{4} \rightarrow 1 .
$$


We now pass to the conformal ball model $B^{4}$ of hyperbolic 4 -space. The vertices of the simplex $\Delta^{4}$ are now $(0,0,0,0),(1-\sqrt{6} / 3,1-\sqrt{6} / 3,1-\sqrt{6} / 3,0)$, $(1-\sqrt{2} / 2,1-\sqrt{2} / 2,0,0),((3-\sqrt{5}) / 4,(3-\sqrt{5}) / 4$, $(3-\sqrt{5}) / 4,(3-\sqrt{5}) / 4)$, and $(1,0,0,0)$.

Let $Q^{4}$ be the regular ideal 24-cell in $B^{4}$ with vertices $( \pm 1,0,0,0),(0, \pm 1,0,0),(0,0, \pm 1,0),(0,0,0, \pm 1)$, and $( \pm 1 / 2, \pm 1 / 2, \pm 1 / 2, \pm 1 / 2)$. The dihedral angles of $Q^{4}$ are all $\pi / 2$. Let $P^{4}$ be the intersection of $Q^{4}$ with the positive hexadecant of $\mathbb{R}^{4}$. Then $P^{4}$ is a noncompact convex polytope with actual vertices $(0,0,0,0),(0,1 / 3,1 / 3,1 / 3),(1 / 3,0,1 / 3,1 / 3)$, $(1 / 3,1 / 3,0,1 / 3),(1 / 3,1 / 3,1 / 3,0)$ and ideal vertices $(1,0,0,0),(0,1,0,0),(0,0,1,0),(0,0,0,1)$, and $(1 / 2,1 / 2,1 / 2,1 / 2)$. Let $T^{4}$ be the 4 -simplex with vertices $(0,0,0,0),(1,0,0,0),(0,1,0,0),(0,0,1,0)$, $(0,0,0,1)$. Call $T^{4}$ a 4 -dimensional corner simplex, with $(0,0,0,0)$ the corner vertex of $T^{4}$, and each side of $T^{4}$ incident with $(0,0,0,0)$ a corner side of $T^{4}$.

The ideal vertices of $P^{4}$ are the vertices of a regular 4-simplex $S^{4}$ in $B^{4}$. The polytope $P^{4}$ is obtained from $S^{4}$ by gluing on to each side of $S^{4}$ a 4-dimensional corner simplex whose corner vertex is an actual vertex of $P^{4}$. Each corner simplex has 4 corner sides and each corner side matches up with a corner side of an adjacent corner simplex to give a total of $4 \cdot 5 / 2=10$ sides of $P^{4}$. The dihedral angles of $P^{4}$ are all $\pi / 2$. Observe that $P^{4}=\Sigma^{4} \Delta^{4}$ and $\Sigma^{4}$ is the group of symmetries of $S^{4}$ and of $P^{4}$.

The Lorentzian matrices that represent the reflections in the sides of $P^{4}$ are

$$
\begin{aligned}
& \left(\begin{array}{rrrrr}
-1 & 0 & 0 & 0 & 0 \\
0 & 1 & 0 & 0 & 0 \\
0 & 0 & 1 & 0 & 0 \\
0 & 0 & 0 & 1 & 0 \\
0 & 0 & 0 & 0 & 1
\end{array}\right), \quad\left(\begin{array}{rrrrr}
1 & 0 & 0 & 0 & 0 \\
0 & -1 & 0 & 0 & 0 \\
0 & 0 & 1 & 0 & 0 \\
0 & 0 & 0 & 1 & 0 \\
0 & 0 & 0 & 0 & 1
\end{array}\right), \\
& \left(\begin{array}{rrrrr}
1 & 0 & 0 & 0 & 0 \\
0 & 1 & 0 & 0 & 0 \\
0 & 0 & -1 & 0 & 0 \\
0 & 0 & 0 & 1 & 0 \\
0 & 0 & 0 & 0 & 1
\end{array}\right), \quad\left(\begin{array}{rrrrr}
1 & 0 & 0 & 0 & 0 \\
0 & 1 & 0 & 0 & 0 \\
0 & 0 & 1 & 0 & 0 \\
0 & 0 & 0 & -1 & 0 \\
0 & 0 & 0 & 0 & 1
\end{array}\right), \\
& \left.\begin{array}{rrrrr}
1 & 0 & 0 & 0 & 0 \\
0 & 1 & 0 & 0 & 0 \\
0 & 0 & -1 & -2 & 2 \\
0 & 0 & -2 & -1 & 2 \\
0 & 0 & -2 & -2 & 3
\end{array}\right), \quad\left(\begin{array}{rrrrr}
1 & 0 & 0 & 0 & 0 \\
0 & -1 & 0 & -2 & 2 \\
0 & 0 & 1 & 0 & 0 \\
0 & -2 & 0 & -1 & 2 \\
0 & -2 & 0 & -2 & 3
\end{array}\right),
\end{aligned}
$$

$$
\begin{aligned}
& \left(\begin{array}{rrrrr}
1 & 0 & 0 & 0 & 0 \\
0 & -1 & -2 & 0 & 2 \\
0 & -2 & -1 & 0 & 2 \\
0 & 0 & 0 & 1 & 0 \\
0 & -2 & -2 & 0 & 3
\end{array}\right), \quad\left(\begin{array}{rrrrr}
-1 & 0 & 0 & -2 & 2 \\
0 & 1 & 0 & 0 & 0 \\
0 & 0 & 1 & 0 & 0 \\
-2 & 0 & 0 & -1 & 2 \\
-2 & 0 & 0 & -2 & 3
\end{array}\right), \\
& \left(\begin{array}{rrrrr}
-1 & 0 & -2 & 0 & 2 \\
0 & 1 & 0 & 0 & 0 \\
-2 & 0 & -1 & 0 & 2 \\
0 & 0 & 0 & 1 & 0 \\
-2 & 0 & -2 & 0 & 3
\end{array}\right), \quad\left(\begin{array}{rrrrr}
-1 & -2 & 0 & 0 & 2 \\
-2 & -1 & 0 & 0 & 2 \\
0 & 0 & 1 & 0 & 0 \\
0 & 0 & 0 & 1 & 0 \\
-2 & -2 & 0 & 0 & 3
\end{array}\right) .
\end{aligned}
$$

These matrices are all in $\Gamma_{2}^{4}$. Now since $\Sigma^{4}$ is a set of coset representatives for $\Gamma_{2}^{4}$ in $\Gamma^{4}$, we have that $P^{4}=\Sigma^{4} \Delta^{4}$ is a fundamental polytope for $\Gamma_{2}^{4}$. We therefore have the following theorem.

Theorem 7. The congruence two subgroup $\Gamma_{2}^{4}$ of the group $\Gamma^{4}$ of integral, positive, Lorentzian $5 \times 5$ matrices is a reflection group with respect to the polytope $P^{4}$.

Let $K^{4}$ be the elementary 2-group of order 16 generated by the 4 reflections in the coordinate hyperplanes of $\mathbb{R}^{4}$. We shall identify $K^{4}$ with the corresponding subgroup of $\Gamma^{4}$ generated by the first 4 matrices in last displayed list of matrices. The next corollary follows immediately from Theorem 7 .

Corollary 3. Every nonidentity element of $\Gamma_{2}^{4}$ of $f$ nite order has order two, every finite subgroup of $\Gamma_{2}^{4}$ is conjugate in $\Gamma^{4}$ to a subgroup of the elementary 2-group $K^{4}$, and there are 5 conjugacy classes of maximal finite subgroups of $\Gamma_{2}^{4}$ in $\Gamma_{2}^{4}$ corresponding to the 5 actual vertices of $P^{4}$.

Let $\Gamma$ be a torsion-free subgroup of $\Gamma_{2}^{4}$ of finite index. Then the group $K^{4}$ acts freely on the set of cosets of $\Gamma$ in $\Gamma_{2}^{4}$ by $g \Gamma \mapsto k g \Gamma$, since $\Gamma$ is torsion-free. Therefore $\left|K^{4}\right|=16$ divides $\left[\Gamma_{2}^{4}: \Gamma\right]$.

Now suppose that $\left[\Gamma_{2}^{4}: \Gamma\right]=16$. Then the regular ideal 24-cell $Q^{4}=K^{4} P^{3}$ is a fundamental polytope for $\Gamma$. The polytope $Q^{4}$ has 24 sides each of which is a regular ideal octahedron.

Let $S$ be a side of $Q^{4}$. Then there is a nonidentity element $g$ of $\Gamma$ that pairs a side $S^{\prime}$ of $Q^{4}$ to $S$. Let $k$ be the element of $K^{4}$ that maps $S^{\prime}$ to $S$, and let $r$ be the reflection in the side $S$. Then $g k r$ leaves $S$ invariant. The elements of $\Gamma_{2}^{4}$ that leave $S$ invariant are the identity, the reflection $r$, the reflections $s$ and $t$ in the two coordinate hyperplanes perpendicular to 
$S$, the $180^{\circ}$ rotations $r s=s r, r t=t r$, and $s t=t s$, and the involution $r$ st. Note that both $s$ and $t$ are in $K^{4}$. We cannot have $g k r$ equal to $r$ or $s r$ or $t r$ or $s t r$, since $g$ has infinite order. Therefore $g k r$ equals 1 or $s$ or $t$ or st. Hence, the side-pairing transformations of $Q^{4}$ in $\Gamma$ are of the form $r k$ where $k$ is in $K^{4}$ and $r$ is the reflection in a side of $Q^{4}$.

Now $\Gamma$ is generated by the side-pairing transformations for $Q^{4}$, and so $\Gamma$ is determined by the sidepairing of $Q^{4}$. The side-pairing for $Q^{4}$ restricts to a side-pairing for each of the 4 copies of $Q^{3}$ obtained from $Q^{4}$ by intersecting $Q^{4}$ with a coordinate hyperplane. Extending the 107 possible side-pairings for $Q^{3}$ that yield an integral, congruence two, hyperbolic 3-manifold on each of the 4 copies of $Q^{3}$ in $Q^{4}$, in a consistent fashion, yields 179625 sidepairings for $Q^{4}$ of the above form. Exactly 137075 of these satisfy the conditions of Poincaré's fundamental polyhedron theorem [Ratcliffe 1994] for the gluing of a complete hyperbolic 4-manifold. These 137075 side-pairings for $Q^{4}$ fall into 5757 equivalence classes under equivalence by a symmetry of $Q^{4}$. The classification of these hyperbolic 4-manifolds is summarized in our next theorem.

Theorem 8. There are, up to isometry, exactly 1171 hyperbolic space-forms $H^{4} / \Gamma$ where $\Gamma$ is a torsionfree subgroup of minimal index in the congruence two subgroup $\Gamma_{2}^{4}$ of the group $\Gamma^{4}$ of integral, positive, Lorentzian $5 \times 5$ matrices. Only 22 of these manifolds are orientable.

Proof. We pass to the conformal ball model $B^{4}$ of hyperbolic 4-space and consider the 137075 sidepairings of the 24-cell $Q^{4}$ that yield an integral, congruence two, hyperbolic 4-manifold. Let $e_{1}, e_{2}, e_{3}, e_{4}$ be the standard basis vectors of $\mathbb{R}^{4}$. Each sidepairing of $Q^{4}$ induces an equivalence relation on the 24 ideal vertices of $Q^{4}$. The equivalence classes are called cycles. The cycle of an ideal vertex $v= \pm e_{i}$ of $Q^{4}$ is either just itself or itself and its antipodal vertex, since an element of $K^{4}$ either fixes $v$ or maps $v$ to $-v$. It turns out that the eight vertices $\pm e_{1}, \pm e_{2}, \pm e_{3}, \pm e_{4}$ fall into either four or five cycles of the form $2,2,2,2$ or $1,1,2,2,2$. The remaining 16 ideal vertices of $Q^{4}$ either form one cycle or divide into two cycles of 8 vertices. The possible vertex cycle structures are $2,2,2,2,16$ or $1,1,2,2,2,16$ or $2,2,2,2,8,8$. Thus all the integral, congruence two, hyperbolic 4-manifolds of minimum volume have 5 or 6 cusps. All the orientable manifolds have 5 cusps.

Let $M^{4}$ be a 5 -cusped manifold. Its side-pairing has a vertex cycle structure 2, 2, 2, 2, 16. Consider a maximum open cusp of $M^{4}$ of vertex cycle order 2. Its boundary is tangent to itself at the point represented by the origin. Let $\pm e_{i}$ be the corresponding two vertices of $Q^{4}$. The horospheres based at $\pm e_{i}$ passing through the origin are also tangent to 24 edges of $Q^{4}$ at their Euclidean midpoints, and these points represent 3 more self-tangency points of the boundary of the cusp. Thus, the boundary of a maximum open cusp of $M^{4}$ of vertex cycle order 2 is tangent to itself at 4 points. The boundary of a maximum open cusp of $M^{4}$ of vertex cycle order 16 is also tangent to itself at 4 points. It turns out that all the self-tangency points of the maximal cusps of $M^{4}$ consist of only 5 points. Each of these 5 points is a self-tangency point of the boundary of only 4 of the maximal cusps. Thus $M^{4}$ has a set of 5 canonical points. The 5 canonical points of $M^{4}$ are represented by the 5 actual vertices of the polytope $P^{4}$.

Each actual vertex of $P^{4}$ is the vertex of a rightangled corner. This suggests a cut and paste operation on $Q^{4}$. Cut $Q^{4}$ along the 4 coordinate hyperplanes into 16 copies of the polytope $P^{4}$. By reassembling a 24-cell around a different corner of $P^{4}$ than the origin, we get possibly 5 different ways to glue up $M^{4}$. We call such a cut and paste operation an inside-out operation on $M^{4}$.

The 5757 equivalence classes of side-pairings of $Q^{4}$ under equivalence by symmetry of $Q^{4}$ split up into 5378 classes with 5 vertex cycles and 379 classes with 6 vertex cycles. By considering canonical points and inside-out operations as in the classification of the 3 -cusped 3-manifolds in the proof of Theorem 5, the 5378 classes of side-pairings, with 5 vertex cycles, represent exactly 1090 isometry classes of 5-cusped hyperbolic 4-manifolds. Table 2 on page 117 lists side-pairings and isometric invariants for the 22 orientable 5-cusped manifolds. Table 3, starting on page 117, lists side-pairings and isometric invariants for the 1068 nonorientable 5-cusped manifolds.

Now let $M^{4}$ be a 6 -cusped manifold. Assume first that it is glued up by a side-pairing of $Q^{4}$ with vertex cycle structure $1,1,2,2,2,16$. Take a maximum 
open cusp of $M^{4}$ of vertex cycle order 1 . Its boundary is tangent to itself at 12 points. The maximum open cusps of vertex cycle order 2 or 16 are tangent to themselves in 4 points as before. Thus, the cusps of vertex cycle order 1 are intrinsically different from the cusps of vertex cycle order 2 or 16 . In fact, the volume of a maximum cusp of vertex cycle order 1 is $16 \sqrt{2} / 3$ whereas the volume of a maximum cusp of vertex cycle order 2 or 16 is $16 / 3$.

Only one of the five self-tangency points of the boundaries of the maximum open cusps of vertex cycle order 2 or 16 is a self-tangency point of the boundaries of all 4 maximum open cusps of vertex cycle order 2 or 16 . Thus $M^{4}$ has a single canonical point represented by one of the actual vertices of $P^{4}$ other than the origin. It turns out that by performing an inside-out operation, $M^{4}$ can also be glued up by a side-pairing of $Q^{4}$ that has the vertex cycle structure $2,2,2,2,8,8$. Here the cusps of vertex cycle order 1 correspond to the cusps of vertex cycle order 8 and the canonical point of $M^{4}$ is represented by the origin. The 12 self-tangency points of a maximum open cusp of vertex cycle order 8 are represented by the centers of the 24 sides of $Q^{4}$.

The 379 equivalence classes of side-pairings of $Q^{4}$ with 6 vertex cycles split up into 298 classes with vertex cycle structure 1, 1, 2, 2, 2, 16 and 81 classes with vertex cycle structure $2,2,2,2,8,8$. These 379 classes of side-pairings of $Q^{4}$ represent exactly 81 isometry classes of 6-cusped hyperbolic 4-manifolds corresponding to the 81 classes of side-pairings with vertex cycle structure $2,2,2,2,8,8$ by the same argument as before. This completes the classification of the integral, congruence two, hyperbolic 4manifolds of minimum volume. Table 4 on page 124 lists side-pairings and isometric invariants for the 81 nonorientable 6-cusped manifolds.

Theorem 9. The congruence two group $\Gamma_{2}^{4}$ has a torsion-free subgroup of index $i$ if and only if $i$ is divisible by 16.

Proof. We have already shown that every torsionfree subgroup of $\Gamma_{2}^{4}$ has index divisible by 16 . From Table 2 we see that $\Gamma_{2}^{3}$ has a torsion-free subgroup $\Gamma$ whose first homology group has an infinite cyclic summand. Therefore $\Gamma$ maps homomorphically onto $\mathbb{Z}$. Hence $\Gamma$ has a subgroup of index $i$ for each positive integer $i$. Therefore $\Gamma_{2}^{4}$ has a torsion-free subgroup of index $16 j$ for each positive integer $j$.

By the Gauss-Bonnet theorem [Gromov 1982; Hopf 1926], the volume of a complete hyperbolic 4-manifold $M$ of finite volume is given by

$$
\operatorname{Vol}(M)=\frac{4 \pi^{2}}{3} \chi(M),
$$

where $\chi(M)$ is the Euler characteristic of $M$. All the 4-manifolds in Theorem 8 have Euler characteristic 1. Therefore all the hyperbolic 4-manifolds in Theorem 8 have minimum volume among all complete hyperbolic 4-manifolds. More generally, the volume spectrum of complete hyperbolic 4-manifolds is given by our last theorem.

Theorem 10. There are complete, orientable, arithmetic, hyperbolic 4-manifolds of finite volume whose Euler characteristic is any given positive integer. Therefore, the volume spectrum of complete hyperbolic 4-manifolds of finite volume is the set of all positive integral multiples of $4 \pi^{2} / 3$.

Proof. The first manifold in Table 2 has a positive first Betti number. Therefore it has an $m$-fold covering for each positive integer $m$. Thus, there are complete, orientable, arithmetic, hyperbolic 4-manifolds of finite volume whose Euler characteristic is any given positive integer $m$.

All the hyperbolic 4-manifolds used to prove Theorem 10 are open. The volume spectrum of closed hyperbolic 4-manifolds is unknown. The closed orientable hyperbolic 4-manifold of smallest known volume is the Davis 120-cell space [Davis 1985] whose Euler characteristic is 26 . We have constructed a closed nonorientable hyperbolic 4-manifold whose Euler characteristic is 17 . This example will be discussed in a future paper.

\section{SIDE-PAIRING CODING}

In this section, we describe the coding that we use to list all the side-pairings of $Q^{n}$ for the integral, congruence two, hyperbolic $n$-manifolds of minimum volume for $n=2,3,4$. Reading this section is necessary only if the reader wants to reconstruct the manifolds in Tables 1, 2, 3, and 4 .

We know that a minimal index torsion-free subgroup of $\Gamma_{2}^{n}$ has as a fundamental domain $Q^{n}$ which 
is an ideal square in dimension 2, a semi-ideal rhombic dodecahedron in dimension 3, or an ideal 24cell in dimension 4 . In each case, the side-pairing maps must be of the form $r k$, where $k$ is in the group $K^{n}$ generated by the reflections in the coordinate hyperplanes, and $r$ is a reflection in a side of $Q^{n}$. Let $r_{1}, r_{2}, \ldots, r_{m}$ be the reflections in the sides of $Q^{n}$ (in a fixed ordering to be specified below). Then to specify one of our manifolds it suffices to list the corresponding sequence $k_{1}, k_{2}, \ldots, k_{m}$ of elements of $K^{n}$ such that the side-pairing maps are $r_{i} k_{i}$ for $i=1,2, \ldots, m$. It will turn out that we don't have to specify quite this much, and that in fact to get a manifold we must have

$k_{4 j+1}=k_{4 j+2}=k_{4 j+3}=k_{4 j+4}$ for $j=0, \ldots,(m / 4)-1$

for a particular ordering of the sides. Thus in the end we will write out only every fourth $k_{i}$ but internally we compute with the complete list of the $k_{i}$.

The elements of $K^{n}$ are given by diagonal $(n+1) \times$ $(n+1)$ matrices of the form

$$
k=\operatorname{diag}\left(a_{1}, \ldots, a_{n}, 1\right) \text { for } a_{i}= \pm 1 .
$$

We encode this $k$ as a single (binary) number

$$
\sum_{i=1}^{n} \frac{\left(1-a_{i}\right)}{2} 2^{i-1}
$$

so that a 1 entry in the matrix is a 0 bit and a -1 entry in the matrix is a 1 bit in the corresponding position in the binary number. Matrix multiplication then corresponds to bitwise mod 2 addition and the binary representation of the number corresponds to an element of $\mathbb{Z}_{2}^{n}$. The action of $k$ in the first coordinate thus corresponds to the least significant bit of the code for $k$. We write the code numbers as a single hexadecimal digit with $\mathrm{A}=10, \mathrm{~B}=11, \ldots$, $\mathrm{F}=15$ in dimension 4 .

In each dimension, in the conformal ball model, each side of the fundamental domain $Q^{n}$ will have ideal vertices on just two of the coordinate axes. Conversely, every pair of ideal vertices of the form $\pm e_{i}$ that are not antipodal to each other will determine a side of $Q^{n}$. We can express this in another way in $\mathbb{R}^{n, 1}$ by noting that the sides of $Q^{n}$ correspond to the vectors of the form $s=\left(a_{1}, \ldots, a_{n}, 1\right)$ such that exactly two of the $a_{i}$ are \pm 1 and the others (if any) are 0 . Such a vector $s$ is a unit normal vector of the corresponding side with respect to the Lorentzian inner product in $\mathbb{R}^{n, 1}$. Note that two sides with unit normal vectors $s$ and $s^{\prime}$ are adjacent if and only if $s \circ s^{\prime}=0$, since the dihedral angle between adjacent sides is $\pi / 2$. Moreover, two sides with unit normal vectors $s$ and $s^{\prime}$ are tangent at infinity if and only if $s \circ s^{\prime}=-1$.

If $r$ is the reflection in the side $S$, and $r k$ is a side-pairing map, then the side mapped to side $S$ by $r k$ is $k S$. One of the conditions for a valid sidepairing must be that if $r k$ is part of the side-pairing, $r$ being the reflection in side $S$ and $r^{\prime}$ is the reflection in the side $S^{\prime}=k S$, then $S^{\prime} \neq S$ and $r^{\prime} k$ is also in the side-pairing, since it is the inverse of $r k$. Each side can only be paired to one of three others (those given by a unit normal vector $s$ with the same zero coordinates) and, whichever one it is paired to, the other two of these sides will then be paired to each other, since the ideal vertices $\pm e_{i}$ can only be mapped to $\pm e_{i}$ by an element of $K^{n}$. This implies that each 2-dimensional coordinate cross-section of the 3-dimensional manifolds will be one of the 2dimensional manifolds and each 3-dimensional coordinate cross-section of the 4-dimensional manifolds will be one of the 3-dimensional manifolds.

It remains to choose a particular sequence of the sides. We list on the next page (bottom left) the vectors $s_{1}, s_{2}, \ldots, s_{m}$ corresponding to the sides of $Q^{n}$. Then the reflections $r_{1}, r_{2}, \ldots, r_{m}$ in these sides are determined and a sequence $k_{1}, k_{2}, \ldots, k_{m}$ of elements of $K^{n}$ (encoded as hexadecimal digits) will define side-pairing maps $r_{i} k_{i}$ giving a manifold. Each of the sets of four sides that must be paired with each other will be taken as a block of consecutive sides in our ordering. The blocks are determined by the nonzero coordinates in the $s_{i}$ and we proceed starting with the first two coordinates nonzero and end with those pairs involving the last 3 coordinates nonzero in a lexicographic fashion.

The reflection $r_{1}$ in the side corresponding to $s_{1}$ is given by

$$
\left(\begin{array}{lll}
-1 & -2 & 2 \\
-2 & -1 & 2 \\
-2 & -2 & 3
\end{array}\right) \text { and }\left(\begin{array}{rrrr}
-1 & -2 & 0 & 2 \\
-2 & -1 & 0 & 2 \\
0 & 0 & 1 & 0 \\
-2 & -2 & 0 & 3
\end{array}\right)
$$

in dimensions 2 and 3, respectively, and by 


$$
\left(\begin{array}{rrrrr}
-1 & -2 & 0 & 0 & 2 \\
-2 & -1 & 0 & 0 & 2 \\
0 & 0 & 1 & 0 & 0 \\
0 & 0 & 0 & 1 & 0 \\
-2 & -2 & 0 & 0 & 3
\end{array}\right) \quad \text { in dimension } 4
$$

The other $r_{i}$ can be obtained as $g^{-1} r_{1} g$ for some matrix $g \in \bar{K}^{n}$ with $g s_{i}=s_{1}$, where $\bar{K}^{n}$ is the group of order $2^{n} n$ ! generated by $K^{n}$ and all the permutation matrices that fix the last coordinate.

Starting with dimension 2 (and using the codes for the elements of $K^{2}$ ), we have $s_{1}=(1,1,1), k_{1}$ is not the identity (coded by 0 ) but can be any of 1,2 , or 3. If $k_{1}=1$, then $k_{1} s_{1}=(-1,1,1)=s_{2}$, and $k_{2}=1$, leaving $s_{3}$ and $s_{4}$ to be paired so $k_{3}=k_{4}=1$ also. If $k_{1}=2$, then $k_{1} s_{1}=(1,-1,1)=s_{3}$, and $k_{3}=2$, leaving $s_{2}$ and $s_{4}$ to be paired so $k_{2}=k_{4}=2$ as well. If $k_{1}=3$, then $k_{1} s_{1}=(-1,-1,1)=s_{4}$, and

$$
\begin{aligned}
& \text { Dimension } 2 \text { Dimension } 4 \\
& s_{1}=(1,1,1) \quad s_{1}=(1,1,0,0,1) \\
& s_{2}=(-1,1,1) \quad s_{2}=(-1,1, \quad 0,0,1) \\
& s_{3}=(1,-1,1) \quad s_{3}=\left(\begin{array}{lll}
1,-1, & 0, & 0,1
\end{array}\right) \\
& s_{4}=(-1,-1,1) \quad s_{5}=(1, \quad 0,1, \quad 0,1) \\
& s_{6}=(-1, \quad 0, \quad 1, \quad 0,1) \\
& s_{7}=(1, \quad 0,-1, \quad 0,1) \\
& \text { Dimension } 3 \quad s_{8}=(-1,0,-1,0,1) \\
& s_{1}=(1,1,0,1) \quad \begin{array}{l}
s_{9}=\left(\begin{array}{lll}
0,1, & 1, & 0,1
\end{array}\right) \\
s_{10}=(0,-1,1,0,1)
\end{array} \\
& s_{2}=(-1, \quad 1, \quad 0,1) \quad s_{11}=\left(\begin{array}{lll}
0, & 1,-1, & 0,1
\end{array}\right) \\
& s_{3}=(1,-1,0,1) \quad s_{12}=(0,-1,-1,0,1)
\end{aligned}
$$

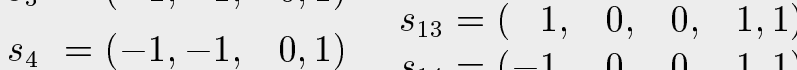

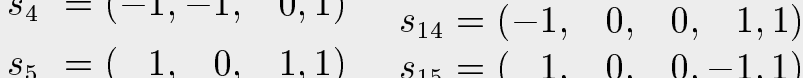

$$
\begin{aligned}
& s_{6}=(-1, \quad 0, \quad 1,1) \quad s_{16}=\left(\begin{array}{lll}
-1, & 0, & 0,-1,1
\end{array}\right) \\
& s_{7}=(1, \quad 0,-1,1) \quad s_{17}=\left(\begin{array}{llll}
0, & 1, & 0, & 1,1
\end{array}\right) \\
& s_{8}=(-1,0,-1,1) \quad s_{18}=\left(\begin{array}{lll}
0,-1, & 0,1,1)
\end{array}\right. \\
& s_{8}=(-1,0,-1,1) \quad s_{19}=\left(\begin{array}{ll}
0, & 1,0,-1,1)
\end{array}\right. \\
& s_{9}=(0,1,1,1) \quad s_{20}=(0,-1, \quad 0,-1,1) \\
& s_{10}=(0,-1,1,1) \quad s_{21}=\left(\begin{array}{lll}
0, & 0,1,1,1)
\end{array}\right. \\
& s_{11}=(0,1,-1,1) \quad s_{22}=\left(\begin{array}{lll}
0, & 0,-1, & 1,1
\end{array}\right) \\
& s_{12}=(0,-1,-1,1) \quad s_{24}=(0,0,-1,-1,1)
\end{aligned}
$$

The vectors $s_{1}, s_{2}, \ldots, s_{m}$ corresponding to the sides of $Q^{n}$ (see preceding page). $k_{4}=3$, and as before $k_{2}=k_{3}=3$ also. Thus the complete codes for the three possible side-pairings of the ideal square $Q^{2}$ are 1111, 2222, and 3333, which we can abbreviate to just 1,2 , and 3 . Now it turns out that side-pairings 1 and 2 are equivalent under a symmetry of $Q^{2}$ and we have two manifolds, 1 being the thrice-punctured sphere and 3 being the twice-punctured projective plane.

In dimension 3 , each two-dimensional coordinate plane cross-section reduces to the two-dimensional case. In the $x y$-plane, $s_{1}=(1,1,0,1)$ cannot be fixed by $k_{1}$ so $k_{1} \neq 0,4$ but $k_{1}$ can be $1,2,3,5,6$, or 7 . Restricting to the 2 -dimensional cross-section, the above reasoning tells us that if $k_{1}$ is 1 or 5 then $k_{2}=k_{1}$ and $k_{3}=k_{4}$ are also either 1 or 5 . If $k_{1}$ is 2 or 6 then $k_{1}=k_{3}$ and $k_{2}=k_{4}$ are also 2 or 6 , and if $k_{1}$ is 3 or 7 then $k_{1}=k_{4}$ and $k_{2}=k_{3}$ is 3 or 7 . Note that maps 5, 6, and 7 reflect the $z$ coordinate whereas the corresponding maps 1,2 , and 3 do not. Similar reasoning applies in the $x z$ - and $y z$-planes. We build a table of the possible side-pairing maps for each block of four sides. In the $x z$ case $s_{5}$ would be fixed by $k_{5}=0$ or 2 , and in the $y z$ case $s_{9}$ would be fixed by $k_{9}=0$ or 1 .

$\begin{array}{ccc}k_{1}-k_{4} & k_{5}-k_{8} & k_{9}-k_{12} \\ 1111 & 1111 & 2222 \\ 1155 & 1133 & 2233 \\ 2222 & 3311 & 3322 \\ 2626 & 3333 & 3333 \\ 3333 & 4444 & 4444 \\ 3773 & 4646 & 4545 \\ 5511 & 5555 & 5454 \\ 5555 & 5775 & 5555 \\ 6262 & 6464 & 6666 \\ 6666 & 6666 & 6776 \\ 7337 & 7557 & 7667 \\ 7777 & 7777 & 7777\end{array}$

There are 3 choices to be made with 12 alternatives each. We need to check which of the $12^{3}=$ 1728 combinations give manifolds. Since sides of the rhombic dodecahedron $Q^{3}$ are at right angles along edges, we need a side-pairing to give cycles of 4 edges. There are 8 actual vertices and these have to form a single cycle under the side-pairing. It turns out that these conditions imply that $k_{1}=k_{2}=k_{3}=k_{4}, k_{5}=k_{6}=k_{7}=k_{8}, k_{9}=k_{10}=k_{11}=k_{12}$, 
and that $k_{1}, k_{5}, k_{9}$ are linearly independent in the $\mathbb{Z}_{2}$-vector space $K^{3}$. Thus a list of only $k_{1}, k_{5}, k_{9}$ will suffice to determine the manifold.

There are then 107 possibilities left for $k_{1}, k_{5}, k_{9}$ :

$134153174237267345375542576 \quad 645712746$ $\begin{array}{llllllllllll}135 & 156 & 175 & 243 & 273 & 346 & 376 & 543 & 612 & 647 & 713 & 753\end{array}$ $136157214245274 \quad 352512546 \quad 613 \quad 652714754$ $137162 \quad 215247276 \quad 354513547614654715756$ 142163216253314357516562615657732762 143164217254315362517564632672735763 146165234256316364532567634673736764 $\begin{array}{llllllllllll}147 & 172 & 235 & 263 & 317 & 367 & 534 & 573 & 637 & 674 & 742 & 765\end{array}$ 152173236265342372537574643675745

This list includes side-pairings that are equivalent under symmetries of $Q^{3}$ as well as different sidepairings yielding isometric manifolds.

The 4-dimensional case is simplified by looking at the four coordinate hyperplane cross-sections. Each cross-section must reduce to one of the above 107 cases. We also have that the choices of side-pairings for the 3-dimensional cross-sections must give the same pairs of sides, that is, the common 2-dimensional cross-sections must be the same. Once we have chosen consistent cross-section side-pairings, the side-pairings of the ideal 24-cell will be determined. Then it becomes a matter of filtering the possible side-pairings according to the 3 -face, 2 -face, and 1-face cycle conditions of Poincaré's fundamental polyhedron theorem, and then reducing the list by symmetries of the 24-cell and hidden isometries between the resulting manifolds. Since each threedimensional cross-section has equal $k_{i}$ for each of the sides $s_{i}$ intersecting a 2-dimensional cross-section, the blocks of 4 sides intersecting each 2-dimensional cross-section will also have equal $k_{i}$ in the 4-dimensional case. Thus a list of only

$$
k_{1}, k_{5}, k_{9}, k_{13}, k_{17}, k_{21}
$$

will suffice to determine the manifold, since

$$
k_{4 i+1}=k_{4 i+2}=k_{4 i+3}=k_{4 i+4} \quad \text { for } i=0,1,2,3,4,5 \text {. }
$$

We now describe in detail how to extract a sidepairing of our fundamental domain $Q^{n}$ from a coded side-pairing. Consider the coded side-pairing for the Borromean rings complement 142 on line 1 of Table 1. It represents the side-pairing

$$
111144442222
$$

for the 12 sides of the rhombic dodecahedron $Q^{3}$. Here

$$
\begin{array}{lll}
1 & \text { represents } & \operatorname{diag}(-1,1,1,1), \\
2 & \text { represents } & \operatorname{diag}(1,-1,1,1), \\
4 & \text { represents } & \operatorname{diag}(1,1,-1,1) .
\end{array}
$$

The set $S_{i}$ of vertices of the side of $Q^{3}$ in $\mathbb{R}^{3}$ whose normal vector in $\mathbb{R}^{3,1}$ is $s_{i}$ is given by

$$
\begin{aligned}
& S_{1}=\left\{(1,0,0),\left(\frac{1}{3}, \frac{1}{3}, \frac{1}{3}\right),\left(\frac{1}{3}, \frac{1}{3},-\frac{1}{3}\right),(0,1,0)\right\} \\
& S_{2}=\left\{(-1,0,0),\left(-\frac{1}{3}, \frac{1}{3}, \frac{1}{3}\right),\left(-\frac{1}{3}, \frac{1}{3},-\frac{1}{3}\right),(0,1,0)\right\} \\
& S_{3}=\left\{(1,0,0),\left(\frac{1}{3},-\frac{1}{3}, \frac{1}{3}\right),\left(\frac{1}{3},-\frac{1}{3},-\frac{1}{3}\right),(0,-1,0)\right\} \\
& S_{4}=\left\{(-1,0,0),\left(-\frac{1}{3},-\frac{1}{3}, \frac{1}{3}\right),\left(-\frac{1}{3},-\frac{1}{3},-\frac{1}{3}\right),(0,-1,0)\right\} \\
& S_{5}=\left\{(1,0,0),\left(\frac{1}{3}, \frac{1}{3}, \frac{1}{3}\right),\left(\frac{1}{3},-\frac{1}{3}, \frac{1}{3}\right),(0,0,1)\right\} \\
& S_{6}=\left\{(-1,0,0),\left(-\frac{1}{3}, \frac{1}{3}, \frac{1}{3}\right),\left(-\frac{1}{3},-\frac{1}{3}, \frac{1}{3}\right),(0,0,1)\right\} \\
& S_{7}=\left\{(1,0,0),\left(\frac{1}{3}, \frac{1}{3},-\frac{1}{3}\right),\left(\frac{1}{3},-\frac{1}{3},-\frac{1}{3}\right),(0,0,-1)\right\} \\
& S_{8}=\left\{(-1,0,0),\left(-\frac{1}{3}, \frac{1}{3},-\frac{1}{3}\right),\left(-\frac{1}{3},-\frac{1}{3},-\frac{1}{3}\right),(0,0,-1)\right\} \\
& S_{9}=\left\{(0,1,0),\left(\frac{1}{3}, \frac{1}{3}, \frac{1}{3}\right),\left(-\frac{1}{3}, \frac{1}{3}, \frac{1}{3}\right),(0,0,1)\right\} \\
& S_{10}=\left\{(0,-1,0),\left(\frac{1}{3},-\frac{1}{3}, \frac{1}{3}\right),\left(-\frac{1}{3},-\frac{1}{3}, \frac{1}{3}\right),(0,0,1)\right\} \\
& S_{11}=\left\{(0,1,0),\left(\frac{1}{3}, \frac{1}{3},-\frac{1}{3}\right),\left(-\frac{1}{3}, \frac{1}{3},-\frac{1}{3}\right),(0,0,-1)\right\} \\
& S_{12}=\left\{(0,-1,0),\left(\frac{1}{3},-\frac{1}{3},-\frac{1}{3}\right),\left(-\frac{1}{3},-\frac{1}{3},-\frac{1}{3}\right),(0,0,-1)\right\}
\end{aligned}
$$

The corresponding ordered sets of vertices of the paired sides are given by

$$
\begin{aligned}
& S_{1}^{\prime}=\left\{(-1,0,0),\left(-\frac{1}{3}, \frac{1}{3}, \frac{1}{3}\right),\left(-\frac{1}{3}, \frac{1}{3},-\frac{1}{3}\right),(0,1,0)\right\} \\
& S_{2}^{\prime}=\left\{(1,0,0),\left(\frac{1}{3}, \frac{1}{3}, \frac{1}{3}\right),\left(\frac{1}{3}, \frac{1}{3},-\frac{1}{3}\right),(0,1,0)\right\} \\
& S_{3}^{\prime}=\left\{(-1,0,0),\left(-\frac{1}{3},-\frac{1}{3}, \frac{1}{3}\right),\left(-\frac{1}{3},-\frac{1}{3},-\frac{1}{3}\right),(0,-1,0)\right\} \\
& S_{4}^{\prime}=\left\{(1,0,0),\left(\frac{1}{3},-\frac{1}{3}, \frac{1}{3}\right),\left(\frac{1}{3},-\frac{1}{3},-\frac{1}{3}\right),(0,-1,0)\right\} \\
& S_{5}^{\prime}=\left\{(1,0,0),\left(\frac{1}{3}, \frac{1}{3},-\frac{1}{3}\right),\left(\frac{1}{3},-\frac{1}{3},-\frac{1}{3}\right),(0,0,-1)\right\} \\
& S_{6}^{\prime}=\left\{(-1,0,0),\left(-\frac{1}{3}, \frac{1}{3},-\frac{1}{3}\right),\left(-\frac{1}{3},-\frac{1}{3},-\frac{1}{3}\right),(0,0,-1)\right\} \\
& S_{7}^{\prime}=\left\{(1,0,0),\left(\frac{1}{3}, \frac{1}{3}, \frac{1}{3}\right),\left(\frac{1}{3},-\frac{1}{3}, \frac{1}{3}\right),(0,0,1)\right\} \\
& S_{8}^{\prime}=\left\{(-1,0,0),\left(-\frac{1}{3}, \frac{1}{3}, \frac{1}{3}\right),\left(-\frac{1}{3},-\frac{1}{3}, \frac{1}{3}\right),(0,0,1)\right\} \\
& S_{9}^{\prime}=\left\{(0,-1,0),\left(\frac{1}{3},-\frac{1}{3}, \frac{1}{3}\right),\left(-\frac{1}{3},-\frac{1}{3}, \frac{1}{3}\right),(0,0,1)\right\} \\
& S_{10}^{\prime}=\left\{(0,1,0),\left(\frac{1}{3}, \frac{1}{3}, \frac{1}{3}\right),\left(-\frac{1}{3}, \frac{1}{3}, \frac{1}{3}\right),(0,0,1)\right\} \\
& S_{11}^{\prime}=\left\{(0,-1,0),\left(\frac{1}{3},-\frac{1}{3},-\frac{1}{3}\right),\left(-\frac{1}{3},-\frac{1}{3},-\frac{1}{3}\right),(0,0,-1)\right\} \\
& S_{12}^{\prime}=\left\{(0,1,0),\left(\frac{1}{3}, \frac{1}{3},-\frac{1}{3}\right),\left(-\frac{1}{3}, \frac{1}{3},-\frac{1}{3}\right),(0,0,-1)\right\}
\end{aligned}
$$

Here we have $S_{i}^{\prime}=k_{i} S_{i}$ where $k_{i}=\operatorname{diag}\left(a_{1}, a_{2}, a_{3}, 1\right)$ acts on $S_{i}$ as a $3 \times 3$ matrix $\operatorname{diag}\left(a_{1}, a_{2}, a_{3}\right)$. 
$S_{1}=\{(1,0,0,0),(1,1,1,1) / 2,(1,1,-1,1) / 2,(1,1,1,-1) / 2,(1,1,-1,-1) / 2,(0,1,0,0)\}$

$S_{2}=\{(-1,0,0,0),(-1,1,1,1) / 2,(-1,1,-1,1) / 2,(-1,1,1,-1) / 2,(-1,1,-1,-1) / 2,(0,1,0,0)\}$

$S_{3}=\{(1,0,0,0),(1,-1,1,1) / 2,(1,-1,-1,1) / 2,(1,-1,1,-1) / 2,(1,-1,-1,-1) / 2,(0,-1,0,0)\}$

$S_{4}=\{(-1,0,0,0),(-1,-1,1,1) / 2,(-1,-1,-1,1) / 2,(-1,-1,1,-1) / 2,(-1,-1,-1,-1) / 2,(0,-1,0,0)\}$

$S_{5}=\{(1,0,0,0),(1,1,1,1) / 2,(1,-1,1,1) / 2,(1,1,1,-1) / 2,(1,-1,1,-1) / 2,(0,0,1,0)\}$

$S_{6}=\{(-1,0,0,0),(-1,1,1,1) / 2,(-1,-1,1,1) / 2,(-1,1,1,-1) / 2,(-1,-1,1,-1) / 2,(0,0,1,0)\}$

$S_{7}=\{(1,0,0,0),(1,1,-1,1) / 2,(1,-1,-1,1) / 2,(1,1,-1,-1) / 2,(1,-1,-1,-1) / 2,(0,0,-1,0)\}$

$S_{8}=\{(-1,0,0,0),(-1,1,-1,1) / 2,(-1,-1,-1,1) / 2,(-1,1,-1,-1) / 2,(-1,-1,-1,-1) / 2,(0,0,-1,0)\}$

$S_{9}=\{(0,1,0,0),(1,1,1,1) / 2,(-1,1,1,1) / 2,(1,1,1,-1) / 2,(-1,1,1,-1) / 2,(0,0,1,0)\}$

$S_{10}=\{(0,-1,0,0),(1,-1,1,1) / 2,(-1,-1,1,1) / 2,(1,-1,1,-1) / 2,(-1,-1,1,-1) / 2,(0,0,1,0)\}$

$S_{11}=\{(0,1,0,0),(1,1,-1,1) / 2,(-1,1,-1,1) / 2,(1,1,-1,-1) / 2,(-1,1,-1,-1) / 2,(0,0,-1,0)\}$

$S_{12}=\{(0,-1,0,0),(1,-1,-1,1) / 2,(-1,-1,-1,1) / 2,(1,-1,-1,-1) / 2,(-1,-1,-1,-1) / 2,(0,0,-1,0)\}$

$S_{13}=\{(1,0,0,0),(1,1,1,1) / 2,(1,-1,1,1) / 2,(1,1,-1,1) / 2,(1,-1,-1,1) / 2,(0,0,0,1)\}$

$S_{14}=\{(-1,0,0,0),(-1,1,1,1) / 2,(-1,-1,1,1) / 2,(-1,1,-1,1) / 2,(-1,-1,-1,1) / 2,(0,0,0,1)\}$

$S_{15}=\{(1,0,0,0),(1,1,1,-1) / 2,(1,-1,1,-1) / 2,(1,1,-1,-1) / 2,(1,-1,-1,-1) / 2,(0,0,0,-1)\}$

$S_{16}=\{(-1,0,0,0),(-1,1,1,-1) / 2,(-1,-1,1,-1) / 2,(-1,1,-1,-1) / 2,(-1,-1,-1,-1) / 2,(0,0,0,-1)\}$

$S_{17}=\{(0,1,0,0),(1,1,1,1) / 2,(-1,1,1,1) / 2,(1,1,-1,1) / 2,(-1,1,-1,1) / 2,(0,0,0,1)\}$

$S_{18}=\{(0,-1,0,0),(1,-1,1,1) / 2,(-1,-1,1,1) / 2,(1,-1,-1,1) / 2,(-1,-1,-1,1) / 2,(0,0,0,1)\}$

$S_{19}=\{(0,1,0,0),(1,1,1,-1) / 2,(-1,1,1,-1) / 2,(1,1,-1,-1) / 2,(-1,1,-1,-1) / 2,(0,0,0,-1)\}$

$S_{20}=\{(0,-1,0,0),(1,-1,1,-1) / 2,(-1,-1,1,-1) / 2,(1,-1,-1,-1) / 2,(-1,-1,-1,-1) / 2,(0,0,0,-1)\}$

$S_{21}=\{(0,0,1,0),(1,1,1,1) / 2,(-1,1,1,1) / 2,(1,-1,1,1) / 2,(-1,-1,1,1) / 2,(0,0,0,1)\}$

$S_{22}=\{(0,0,-1,0),(1,1,-1,1) / 2,(-1,1,-1,1) / 2,(1,-1,-1,1) / 2,(-1,-1,-1,1) / 2,(0,0,0,1)\}$

$S_{23}=\{(0,0,1,0),(1,1,1,-1) / 2,(-1,1,1,-1) / 2,(1,-1,1,-1) / 2,(-1,-1,1,-1) / 2,(0,0,0,-1)\}$

$S_{24}=\{(0,0,-1,0),(1,1,-1,-1) / 2,(-1,1,-1,-1) / 2,(1,-1,-1,-1) / 2,(-1,-1,-1,-1) / 2,(0,0,0,-1)\}$

$S_{1}^{\prime}=\{(-1,0,0,0),(-1,1,1,1) / 2,(-1,1,-1,1) / 2,(-1,1,1,-1) / 2,(-1,1,-1,-1) / 2,(0,1,0,0)\}$

$S_{2}^{\prime}=\{(1,0,0,0),(1,1,1,1) / 2,(1,1,-1,1) / 2,(1,1,1,-1) / 2,(1,1,-1,-1) / 2,(0,1,0,0)\}$

$S_{3}^{\prime}=\{(-1,0,0,0),(-1,-1,1,1) / 2,(-1,-1,-1,1) / 2,(-1,-1,1,-1) / 2,(-1,-1,-1,-1) / 2,(0,-1,0,0)\}$

$S_{4}^{\prime}=\{(1,0,0,0),(1,-1,1,1) / 2,(1,-1,-1,1) / 2,(1,-1,1,-1) / 2,(1,-1,-1,-1) / 2,(0,-1,0,0)\}$

$S_{5}^{\prime}=\{(1,0,0,0),(1,1,-1,1) / 2,(1,-1,-1,1) / 2,(1,1,-1,-1) / 2,(1,-1,-1,-1) / 2,(0,0,-1,0)\}$

$S_{6}^{\prime}=\{(-1,0,0,0),(-1,1,-1,1) / 2,(-1,-1,-1,1) / 2,(-1,1,-1,-1) / 2,(-1,-1,-1,-1) / 2,(0,0,-1,0)\}$

$S_{7}^{\prime}=\{(1,0,0,0),(1,1,1,1) / 2,(1,-1,1,1) / 2,(1,1,1,-1) / 2,(1,-1,1,-1) / 2,(0,0,1,0)\}$

$S_{8}^{\prime}=\{(-1,0,0,0),(-1,1,1,1) / 2,(-1,-1,1,1) / 2,(-1,1,1,-1) / 2,(-1,-1,1,-1) / 2,(0,0,1,0)\}$

$S_{9}^{\prime}=\{(0,-1,0,0),(1,-1,1,1) / 2,(-1,-1,1,1) / 2,(1,-1,1,-1) / 2,(-1,-1,1,-1) / 2,(0,0,1,0)\}$

$S_{10}^{\prime}=\{(0,1,0,0),(1,1,1,1) / 2,(-1,1,1,1) / 2,(1,1,1,-1) / 2,(-1,1,1,-1) / 2,(0,0,1,0)\}$

$S_{11}^{\prime}=\{(0,-1,0,0),(1,-1,-1,1) / 2,(-1,-1,-1,1) / 2,(1,-1,-1,-1) / 2,(-1,-1,-1,-1) / 2,(0,0,-1,0)\}$

$S_{12}^{\prime}=\{(0,1,0,0),(1,1,-1,1) / 2,(-1,1,-1,1) / 2,(1,1,-1,-1) / 2,(-1,1,-1,-1) / 2,(0,0,-1,0)\}$

$S_{13}^{\prime}=\{(1,0,0,0),(1,1,1,-1) / 2,(1,-1,1,-1) / 2,(1,1,-1,-1) / 2,(1,-1,-1,-1) / 2,(0,0,0,-1)\}$

$S_{14}^{\prime}=\{(-1,0,0,0),(-1,1,1,-1) / 2,(-1,-1,1,-1) / 2,(-1,1,-1,-1) / 2,(-1,-1,-1,-1) / 2,(0,0,0,-1)\}$

$S_{15}^{\prime}=\{(1,0,0,0),(1,1,1,1) / 2,(1,-1,1,1) / 2,(1,1,-1,1) / 2,(1,-1,-1,1) / 2,(0,0,0,1)\}$

$S_{16}^{\prime}=\{(-1,0,0,0),(-1,1,1,1) / 2,(-1,-1,1,1) / 2,(-1,1,-1,1) / 2,(-1,-1,-1,1) / 2,(0,0,0,1)\}$

$S_{17}^{\prime}=\{(0,-1,0,0),(-1,-1,1,-1) / 2,(1,-1,1,-1) / 2,(-1,-1,-1,-1) / 2,(1,-1,-1,-1) / 2,(0,0,0,-1)\}$

$S_{18}^{\prime}=\{(0,1,0,0),(-1,1,1,-1) / 2,(1,1,1,-1) / 2,(-1,1,-1,-1) / 2,(1,1,-1,-1) / 2,(0,0,0,-1)\}$

$S_{19}^{\prime}=\{(0,-1,0,0),(-1,-1,1,1) / 2,(1,-1,1,1) / 2,(-1,-1,-1,1) / 2,(1,-1,-1,1) / 2,(0,0,0,1)\}$

$S_{20}^{\prime}=\{(0,1,0,0),(-1,1,1,1) / 2,(1,1,1,1) / 2,(-1,1,-1,1) / 2,(1,1,-1,1) / 2,(0,0,0,1)\}$

$S_{21}^{\prime}=\{(0,0,-1,0),(-1,1,-1,-1) / 2,(1,1,-1,-1) / 2,(-1,-1,-1,-1) / 2,(1,-1,-1,-1) / 2,(0,0,0,-1)\}$

$S_{22}^{\prime}=\{(0,0,1,0),(-1,1,1,-1) / 2,(1,1,1,-1) / 2,(-1,-1,1,-1) / 2,(1,-1,1,-1) / 2,(0,0,0,-1)\}$

$S_{23}^{\prime}=\{(0,0,-1,0),(-1,1,-1,1) / 2,(1,1,-1,1) / 2,(-1,-1,-1,1) / 2,(1,-1,-1,1) / 2,(0,0,0,1)\}$

$S_{24}^{\prime}=\{(0,0,1,0),(-1,1,1,1) / 2,(1,1,1,1) / 2,(-1,-1,1,1) / 2,(1,-1,1,1) / 2,(0,0,0,1)\}$ 


$\begin{array}{ccccccc}N & S P & S & H_{1} & H_{2} & H_{3} & L T \\ 1 & 1428 B D & 16 & 330 & 700 & 4 & \text { AAABF } \\ 2 & 14278 D & 16 & 240 & 600 & 4 & \text { AABBF } \\ 3 & 1477 B 8 & 16 & 240 & 600 & 4 & \text { AABBF } \\ 4 & 1477 \mathrm{BE} & 16 & 240 & 600 & 4 & \mathrm{AABBF} \\ 5 & 1478 \mathrm{ED} & 16 & 240 & 600 & 4 & \mathrm{AABBF} \\ 6 & 14278 \mathrm{E} & 16 & 240 & 600 & 4 & \mathrm{ABBBF} \\ 7 & 142 \mathrm{DBE} & 48 & 150 & 500 & 4 & \mathrm{ABBBF}\end{array}$

$\begin{array}{rcccccc}N & S P & S & H_{1} & H_{2} & H_{3} & L T \\ 8 & 1427 \mathrm{BD} & 16 & 150 & 500 & 4 & \mathrm{ABBBF} \\ 9 & 1477 \mathrm{~EB} & 16 & 150 & 500 & 4 & \mathrm{ABBBF} \\ 10 & 1477 \mathrm{ED} & 16 & 150 & 500 & 4 & \mathrm{ABBBF} \\ 11 & 1478 \mathrm{~EB} & 16 & 150 & 500 & 4 & \mathrm{ABBBF} \\ 12 & 147 \mathrm{BDE} & 16 & 150 & 500 & 4 & \mathrm{ABBBF} \\ 13 & 14 \mathrm{~B} 8 \mathrm{ED} & 16 & 150 & 500 & 4 & \mathrm{ABBBF} \\ 14 & \text { 1427BE } & 16 & 150 & 500 & 4 & \mathrm{BBBBF} \\ 15 & 1477 \mathrm{DE} & 16 & 150 & 500 & 4 & \mathrm{BBBBF}\end{array}$

$\begin{array}{ccccccc}N & S P & S & H_{1} & H_{2} & H_{3} & L T \\ 16 & \text { 14B7E8 } & 16 & 060 & 400 & 4 & \mathrm{BBBBF} \\ 17 & \text { 14B7ED } & 16 & 060 & 400 & 4 & \mathrm{BBBBF} \\ 18 & \text { 14BDE7 } & 16 & 060 & 400 & 4 & \mathrm{BBBBF} \\ 19 & \text { 14B7DE } & 16 & 060 & 400 & 4 & \mathrm{BBFFF} \\ 20 & \text { 14B8E7 } & 16 & 051 & 400 & 4 & \mathrm{ABFFF} \\ 21 & \text { 14BD7E } & 16 & 051 & 400 & 4 & \mathrm{ABFFF} \\ 22 & \text { 17BE8D } & 16 & 051 & 400 & 4 & \mathrm{ABFFF}\end{array}$

TABLE 2. Orientable, 5 cusped, minimal volume, integral, congruence 2, hyperbolic 4-manifolds.

Now take the first orientable 4-manifold in Table 2 above. The hexadecimal digits in the column headed $S P$ are interpreted as follows:

$$
\begin{array}{rll}
1 & \text { represents } & \operatorname{diag}(-1,1,1,1,1), \\
2 & \text { represents } & \operatorname{diag}(1,-1,1,1,1), \\
4 & \text { represents } & \operatorname{diag}(1,1,-1,1,1), \\
8 & \text { represents } & \operatorname{diag}(1,1,1,-1,1), \\
\text { B } & \text { represents } & \operatorname{diag}(-1,-1,1,-1,1), \\
\text { D } & \text { represents } & \operatorname{diag}(-1,1,-1,-1,1),
\end{array}
$$

and the code 1428BD represents the side-pairing

1111444422228888BBBBDDDD for the 24 sides of the 24-cell $Q^{4}$. The set $S_{i}$ of vertices of the side of $Q^{4}$ whose normal vector is $s_{i}$ is given in the upper half of page 116. The corresponding ordered sets of the vertices of the paired sides are given in the lower half of the same page. Here we have $S_{i}^{\prime}=k_{i} S_{i}$ where $k_{i}=\operatorname{diag}\left(a_{1}, a_{2}, a_{3}, a_{4}, 1\right)$ acts on $S_{i}$ as a $4 \times 4$ matrix $\operatorname{diag}\left(a_{1}, a_{2}, a_{3}, a_{4}\right)$.

\section{TABLES}

Tables 2-4 list side-pairings and isometric invariants of all the congruence two 24-cell manifolds. In each table, $N$ is the row number. The column

\begin{abstract}
$N \quad S P \quad S \quad H_{1} \quad H_{2} \quad H_{3} \quad L T$
23 1569A4 $32420 \quad 620 \quad 2 \quad$ AAGGH

24 134B2E $16 \quad 420 \quad 620 \quad 2$ AAGHH

25 134B3E $16 \quad 420 \quad 620 \quad 2$ AAGHH

26 13483D $16 \quad 420 \quad 620 \quad 2$ AAGHJ

27 1348BD $16 \quad 420 \quad 620 \quad 2$ AAGHJ

$28 \quad 13492 \mathrm{C} \quad 16 \quad 420 \quad 620 \quad 2$ AAHHI

29 1349AC $16 \quad 420620 \quad 2$ AAHHI

30 1429AC $48 \quad 420 \quad 620 \quad 2 \quad$ AFGGG

$31 \quad 13 C 835 \quad 16 \quad 420 \quad 530 \quad 1 \quad$ AGGGJ

$32 \quad 13482 \mathrm{C} \quad 16 \quad 420 \quad 530 \quad 1 \quad$ AGGHH

33 1348AC $16 \quad 420 \quad 530 \quad 1$ AGGHJ

34 1348BC $16 \quad 420530 \quad 1$ AGHHI

$\begin{array}{lllllll}35 & 146928 & 64 & 420 & 440 & 0 & \text { GGGGH }\end{array}$

36 1468AF $32 \quad 330 \quad 610 \quad 3 \quad$ AAAJJ

37 156F8C $32 \quad 330 \quad 610 \quad 3 \quad$ AABJJ

$\begin{array}{lllllll}38 & 143 B D 8 & 16 & 330 & 610 & 3 & \text { ABFGH }\end{array}$

39 14378D $16 \quad 330 \quad 610 \quad 3 \quad$ ABFHH

40 143CF9 $16 \quad 330520 \quad 2$ AAGGJ

41 13FF8A 323305202 AAGHH

42 13482D $16 \quad 330520 \quad 2$ AAGHJ

43 1348FD $16 \quad 330520 \quad 2$ AAGHJ

\begin{tabular}{|c|c|c|c|c|c|c|}
\hline & $S P$ & $S$ & $H_{1}$ & $H_{2}$ & $H_{3}$ & $L T$ \\
\hline & $34 \mathrm{~A} 3 \mathrm{~F}$ & 16 & 330 & 520 & 2 & AAHIJ \\
\hline & 1369A4 & 16 & 330 & 520 & 2 & AIIJ \\
\hline & $156 \mathrm{~A} 9 \mathrm{C}$ & 16 & 330 & 520 & 2 & ABGGH \\
\hline & 13D834 & 16 & 330 & 520 & 2 & ABGGJ \\
\hline & $136 \mathrm{~F} 8 \mathrm{~A}$ & 32 & 330 & 520 & 2 & ABGHH \\
\hline 5 & $134 \mathrm{~B} 6 \mathrm{E}$ & 16 & 330 & 520 & 2 & ABGHH \\
\hline 55 & 134B7E & 16 & 330 & 520 & 2 & ABGHH \\
\hline 56 & 13D935 & 16 & 330 & 520 & 2 & ABGHI \\
\hline 57 & $13482 E$ & 16 & 330 & 520 & 2 & ABGHJ \\
\hline 58 & 13487D & 16 & 30 & 520 & 2 & ABGHJ \\
\hline 59 & $1348 A D$ & 16 & 330 & 520 & 2 & ABGHJ \\
\hline 60 & 134B3C & 16 & 30 & 520 & 2 & ABGHJ \\
\hline 61 & 136DA8 & 16 & 330 & 520 & 2 & ABGHJ \\
\hline 62 & $1439 \mathrm{AC}$ & 16 & 330 & 520 & 2 & ABGHJ \\
\hline 63 & $143 B 9 C$ & 16 & 330 & 520 & 2 & ABGHJ \\
\hline 64 & $13483 F$ & 16 & 330 & 520 & 2 & ABGJJ \\
\hline 65 & $13496 \mathrm{C}$ & 16 & 330 & 520 & 2 & ABHHI \\
\hline 66 & 1349BC & 16 & 330 & 520 & 2 & ABHHI \\
\hline 67 & $134 \mathrm{~A} 2 \mathrm{C}$ & 16 & 330 & 520 & 2 & ABHIJ \\
\hline 68 & $1368 \mathrm{~A} 4$ & 16 & 330 & 520 & 2 & AFGGI \\
\hline 69 & 1347B8 & 16 & 330 & 520 & 2 & AFGII \\
\hline 70 & $1437 \mathrm{C} 9$ & 16 & 330 & 520 & 2 & BFGGH \\
\hline 71 & 13ЕВ34 & 16 & 330 & 430 & 1 & AGGGH \\
\hline 72 & 13EB64 & 16 & 330 & 430 & 1 & AGGGH \\
\hline 73 & 13582D & 16 & 0 & 430 & & $\mathrm{AG}$ \\
\hline 74 & $35 B 3 F$ & 16 & 30 & 430 & 1 & \\
\hline
\end{tabular}

44 136B84 $16 \quad 330520 \quad 2$ AAGIJ

\begin{tabular}{|c|c|c|c|c|c|c|}
\hline & $S P$ & $S$ & $H_{1}$ & $H_{2}$ & $H_{3}$ & $L T$ \\
\hline 75 & 13 & 16 & 330 & 43 & 1 & \\
\hline 76 & 13C8B4 & 16 & 330 & 430 & 1 & AGGH I \\
\hline 77 & 13СВ36 & 16 & 330 & 430 & 1 & AGGHI \\
\hline 78 & $13 \mathrm{EFCA}$ & 16 & 330 & 430 & 1 & AGGHI \\
\hline 79 & 157B9D & 16 & 330 & 430 & 1 & AGGH I \\
\hline 80 & $13483 E$ & 16 & 330 & 430 & 1 & AGGHJ \\
\hline 81 & 1348FC & 16 & 330 & 430 & 1 & AGGHJ \\
\hline 82 & $134 \mathrm{~B} 2 \mathrm{C}$ & 16 & 330 & 430 & 1 & AGGHJ \\
\hline 83 & $13583 \mathrm{C}$ & 16 & 330 & 430 & 1 & AGGHJ \\
\hline 84 & 135B2E & 16 & 330 & 430 & 1 & AGGHJ \\
\hline 85 & 136B2E & 16 & 330 & 430 & 1 & AGGHJ \\
\hline 86 & $136 \mathrm{~F} 2 \mathrm{~A}$ & 16 & 330 & 430 & 1 & AGGHJ \\
\hline 87 & 13EE64 & 16 & 330 & 430 & 1 & AGGHJ \\
\hline 88 & $13 \mathrm{C} 875$ & 16 & 330 & 430 & 1 & AGGIJ \\
\hline 89 & 13EA35 & 16 & 330 & 430 & 1 & AGGIJ \\
\hline 90 & 134B3D & 16 & 330 & 430 & 1 & AGGJJ \\
\hline 91 & $136 \mathrm{D} 28$ & 16 & 330 & 430 & 1 & AGGJJ \\
\hline 92 & 1348EC & 16 & 330 & 430 & 1 & AGHHI \\
\hline 93 & 13593D & 16 & 330 & 430 & 1 & AGHHI \\
\hline 94 & $135 \mathrm{~A} 2 \mathrm{~F}$ & 16 & 330 & 430 & 1 & AGHHI \\
\hline 95 & $136 \mathrm{CA} 8$ & 16 & 330 & 430 & 1 & AGHHI \\
\hline 96 & 13EFC4 & 16 & 330 & 430 & 1 & AGHHI \\
\hline 97 & 147B9C & 16 & 330 & 430 & 1 & AGHHJ \\
\hline 98 & $1347 \mathrm{~A} 8$ & 16 & 330 & 430 & 1 & AGHIJ \\
\hline 99 & $13486 \mathrm{C}$ & 16 & 330 & 430 & 1 & AGHIJ \\
\hline 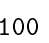 & $13487 \mathrm{C}$ & 16 & 30 & 430 & 1 & AGH \\
\hline
\end{tabular}

45 13ED28 $16 \quad 330520 \quad 2$ AAGIJ

$46 \quad 13493 \mathrm{C} \quad 16 \quad 330520 \quad 2$ AAHHI

47 1349EC $16 \quad 330520 \quad 2$ AAHHI

48 134A2F $16 \quad 330520 \quad 2$ AAHIJ
\end{abstract}

TABLE 3 (start). Non-orientable, 5 cusped, minimal volume, integral, congruence 2, hyperbolic 4-manifolds. 


\section{$\begin{array}{lllllll}N & S P & S & H_{1} & H_{2} & H_{3} & L T\end{array}$}

101 13492E $16 \quad 330 \quad 430 \quad 1$ AGHIJ $102 \quad 13592 \mathrm{C} \quad 16 \quad 330 \quad 430 \quad 1$ AGHIJ 103 135A3E $16 \quad 330430 \quad 1$ AGHIJ 104 1367A8 $16 \quad 330 \quad 430 \quad 1$ AGHIJ 105 1368AE $16 \quad 330 \quad 430 \quad 1$ AGHIJ $106 \quad 13692 \mathrm{C} 16 \quad 330 \quad 430 \quad 1$ AGHIJ 107 1359AC $16 \quad 330430 \quad 1$ AGIIJ 108 1349BD $16 \quad 330 \quad 430 \quad 1$ AGIJJ 109 1369AE $16 \quad 330 \quad 430 \quad 1$ AHHHI $\begin{array}{lllllll}110 & 1347 B 9 & 16 & 330 & 430 & 1 & \text { AHIIJ }\end{array}$ 111 1349AD $16 \quad 330 \quad 430 \quad 1$ AHIIJ $112 \quad 13 C 874 \quad 16 \quad 330 \quad 430 \quad 1 \quad$ BGGGH 113 1358AC $16 \quad 330 \quad 430 \quad 1$ BGGGJ 114 13EECA $16 \quad 330 \quad 430 \quad 1$ BGGGJ $115 \quad 13682 \mathrm{E} \quad 16 \quad 330 \quad 430 \quad 1 \quad$ BGGHH $\begin{array}{lllllll}116 & 136 \mathrm{C} 2 \mathrm{~A} & 16 & 330 & 430 & 1 & \mathrm{BGGHH}\end{array}$ 117 1359BD $16 \quad 330 \quad 430 \quad 1 \quad$ BGGHI 118 136E28 $16 \quad 330 \quad 430 \quad 1 \quad$ BGGHJ 119 13EEC4 $16 \quad 330 \quad 430 \quad 1 \quad$ BGGHJ $\begin{array}{lllllll}120 & 13 C 837 & 16 & 330 & 430 & 1 & \text { BGGIJ }\end{array}$ 121 157A9C $16 \quad 330 \quad 430 \quad 1 \quad$ BGHHH 122 136A2C $16 \quad 330 \quad 430 \quad 1 \quad$ BGHHI 123 153A9C $16 \quad 330 \quad 430 \quad 1$ BGHHI 124 1539AD $16 \quad 330 \quad 430 \quad 1$ BGHHJ $125 \quad 143$ AC9 $16 \quad 330 \quad 430 \quad 1 \quad$ FGGGH 126 1539BD $16 \quad 330 \quad 430 \quad 1 \quad$ FGGGH 127 1439BC $16 \quad 330 \quad 430 \quad 1 \quad$ FGGHH 128 136B8C $16 \quad 330 \quad 421 \quad 1 \quad$ AGHHH $129 \begin{array}{lllllll}13678 A & 16 & 330 & 421 & 1 & \text { AGHIJ }\end{array}$ $130 \begin{array}{lllllll}13 C B 35 & 16 & 330 & 340 & 0 & \text { GGGGJ }\end{array}$ 131 13583D $16 \quad 330 \quad 340 \quad 0 \quad$ GGGHH $132 \quad 136$ F28 $16 \quad 330 \quad 340 \quad 0 \quad$ GGGHH $\begin{array}{lllllll}133 & 13 C 836 & 16 & 330 & 340 & 0 & \text { GGGHI }\end{array}$ 134 135B3E $16 \quad 330 \quad 340 \quad 0 \quad$ GGGHJ $135136 \mathrm{C} 28 \quad 16 \quad 330 \quad 340 \quad 0 \quad$ GGGHJ $136 \quad 136 \mathrm{D} 2 \mathrm{~A} \quad 16 \quad 330 \quad 340 \quad 0 \quad$ GGGHJ $\begin{array}{lllllll}137 & 13 C 935 & 16 & 330 & 340 & 0 & \text { GGGIJ }\end{array}$ 138 136E2A $16 \quad 330 \quad 340 \quad 0 \quad$ GGGJJ 139 13EF64 $16 \quad 330 \quad 340 \quad 0 \quad$ GGHHH $140 \quad 135 \mathrm{~A} 3 \mathrm{~F} \quad 16 \quad 330 \quad 340 \quad 0 \quad$ GGHHI 141 13EA64 $16 \quad 330340 \quad 0 \quad$ GGHHI 142 1358AD $16330340 \quad 0 \quad$ GGHHJ $14313593 \mathrm{C} \quad 16 \quad 330 \quad 340 \quad 0 \quad$ GGHIJ 144 156B9D $16 \quad 330 \quad 340 \quad 0 \quad$ GHHHI 145 1359BC $16 \quad 330340 \quad 0 \quad$ GHIJ J 146 135B2F $16 \quad 330 \quad 331 \quad 0 \quad$ GGGHH 147 136B2C $16 \quad 330331 \quad 0 \quad$ GGGHH $\begin{array}{lllllll}148 & 13582 \mathrm{C} & 16 & 330 & 331 & 0 & \text { GGGHJ }\end{array}$ $14913682 \mathrm{C} \quad 16 \quad 330 \quad 331 \quad 0 \quad$ GGGHJ $\begin{array}{lllllll}150 & 13592 D & 16 & 330 & 331 & 0 & \text { GGHHI }\end{array}$ $\begin{array}{lllllll}151 & 13692 \mathrm{E} & 16 & 330 & 331 & 0 & \text { GGHHI }\end{array}$ $\begin{array}{lllllll}152 & 1358 B C & 16 & 330 & 331 & 0 & \text { GGHHJ }\end{array}$ $\begin{array}{lllllll}153 & 13 C 8 B 5 & 16 & 330 & 331 & 0 & \text { GGHHJ }\end{array}$ 154 135A2E $16 \quad 330 \quad 331 \quad 0 \quad$ GGHIJ 155 136A2E $16 \quad 3303310$ GGHIJ
$\begin{array}{lllllll}N & S P & S & H_{1} & H_{2} & H_{3} & L T\end{array}$

156 1359AD $16 \quad 330331 \quad 0 \quad$ GHHHI 157 3579BF $32 \quad 250 \quad 430 \quad 2$ ABGII $158 \quad 1437 F 9 \quad 16 \quad 240 \quad 510 \quad 3 \quad$ ABBGJ $159 \quad 14378 \mathrm{E} \quad 16 \quad 240 \quad 510 \quad 3 \quad$ ABBJJ 160 143DF8 $16240510 \quad 3 \quad$ ABFGH $161 \quad 14779 \mathrm{C} \quad 16 \quad 240 \quad 510 \quad 3 \quad \mathrm{ABFGH}$ $16214 F 7 C 9 \quad 16 \quad 240 \quad 510 \quad 3 \quad$ ABFGJ 163 14386D $16240510 \quad 3 \quad$ ABFHH 164 143E8D $16240510 \quad 3 \quad$ ABFHH 165 1437FA $16 \quad 240 \quad 510 \quad 3 \quad$ ABFHI $\begin{array}{lllllll}166 & 14279 \mathrm{C} & 16 & 240 & 510 & 3 & \mathrm{BBFGG}\end{array}$ $167 \quad 1427 \mathrm{AC} \quad 16 \quad 240 \quad 510 \quad 3 \quad \mathrm{BBFGG}$ 168 1437D8 $16 \quad 240510 \quad 3 \quad$ BBFGH 169 143D68 $16240510 \quad 3 \quad$ BBFGH 170 1CFF38 $32 \quad 240 \quad 420 \quad 2$ AAGJJ $171 \quad 153$ CF4 $32 \quad 240 \quad 420 \quad 2$ AAHII 172 13D864 $16 \quad 240 \quad 420 \quad 2 \quad$ ABGGJ 173 143DE8 $16240 \quad 4202$ ABGGJ 174 13DB37 $16 \quad 240 \quad 420 \quad 2$ ABGHI 175 13486D $16 \quad 240 \quad 420 \quad 2 \quad$ ABGHJ 176 1348ED $16 \quad 240 \quad 420 \quad 2 \quad$ ABGHJ 177 136B3C $16 \quad 240 \quad 420 \quad 2$ ABGHJ 178 136F38 $16 \quad 240 \quad 420 \quad 2 \quad$ ABGHJ 179 13D8B4 $16 \quad 240 \quad 420 \quad 2 \quad$ ABGHJ 180 13EE84 $16 \quad 240 \quad 420 \quad 2 \quad$ ABGHJ 181 13EED4 $16 \quad 240 \quad 420 \quad 2$ ABGHJ $1821477 B 9 \quad 16 \quad 240 \quad 420 \quad 2 \quad$ ABGHJ 183 1479BE 162404202 ABGHJ $184 \quad 1347 \mathrm{E} 8 \quad 16 \quad 240 \quad 420 \quad 2 \quad$ ABGIJ 185 136B8D $16 \quad 240 \quad 420 \quad 2$ ABGIJ 186 13EEDB $16240 \quad 420 \quad 2$ ABGIJ $\begin{array}{lllllll}187 & 14 B C 79 & 16 & 240 & 420 & 2 & \text { ABGIJ }\end{array}$ 188 134B2D $16 \quad 240 \quad 420 \quad 2$ ABGJJ 189 13683F $16 \quad 240 \quad 420 \quad 2 \quad$ ABGJJ 190 1368AF $16 \quad 240 \quad 420 \quad 2 \quad$ ABGJJ 191 136D3A $16 \quad 240 \quad 420 \quad 2$ ABGJJ 192 13DD64 $16240 \quad 420 \quad 2 \quad$ ABGJJ $193 \quad 143 B 68 \quad 16240 \quad 420 \quad 2 \quad$ ABGJJ 194 13497C $16 \quad 240 \quad 420 \quad 2 \quad$ ABHHI 195 1349FC $16 \quad 240 \quad 420 \quad 2$ ABHHI 196 136CA9 $16 \quad 240 \quad 420 \quad 2$ ABHII 197 13D975 $16 \quad 240420 \quad 2 \quad$ ABHII 198 13493E $16 \quad 240 \quad 420 \quad 2$ ABHIJ 199 134A7F $16 \quad 240 \quad 420 \quad 2$ ABHIJ 200 13693E $16 \quad 240 \quad 420 \quad 2$ ABHIJ 201 136C3B 162404202 ABHIJ 202 136CA4 $16 \quad 240420 \quad 2$ ABHIJ 203 13DCF4 $16240 \quad 420 \quad 2$ ABHIJ 204 156A3C $16 \quad 240420 \quad 2$ ABHIJ 205 156DAE 162404202 ABHIJ 206 1347F9 $16240420 \quad 2$ ABIIJ 207 13D9A4 $16240420 \quad 2$ ABIIJ 208 13DA36 $16 \quad 240 \quad 420 \quad 2$ ABIIJ 209 13EA75 $16 \quad 240420 \quad 2$ ABIIJ $210 \quad 13492 F \quad 16240 \quad 420 \quad 2 \quad$ ABIJJ
$\begin{array}{lllllll}N & S P & S & H_{1} & H_{2} & H_{3} & L T\end{array}$ 211 134A3D $16 \quad 240 \quad 420 \quad 2$ ABIJJ $\begin{array}{lllllll}212 & 136 \mathrm{ABD} & 16 & 240 & 420 & 2 & \text { ABIJJ }\end{array}$ $\begin{array}{lllllll}213 & 136 E 39 & 16 & 240 & 420 & 2 & \text { ABIJJ }\end{array}$ 214 136E84 $16240420 \quad 2$ ABIJJ $\begin{array}{lllllll}215 & 13 E E 75 & 16 & 240 & 420 & 2 & \text { ABIJJ }\end{array}$ 216 1429FA $16 \quad 240 \quad 420 \quad 2 \quad$ AFGGG 217 136F84 $16 \quad 240 \quad 420 \quad 2$ AFGHI 218 1439FA $16 \quad 240 \quad 420 \quad 2$ AFGHI 219 13487E $16 \quad 240 \quad 420 \quad 2 \quad$ AFGHJ 220 134B6C $16 \quad 240 \quad 420 \quad 2 \quad$ AFGHJ 221 143B9E $16 \quad 240 \quad 420 \quad 2$ AFGHJ 222 136DA4 $16240 \quad 420 \quad 2$ AFGIJ 223 156F9C $16 \quad 240 \quad 420 \quad 2$ AFGIJ 224 134B7D $16240 \quad 420 \quad 2 \quad$ AFGJJ $225 \quad 1347 \mathrm{FC} \quad 16 \quad 240 \quad 420 \quad 2$ AFHII $\begin{array}{lllllll}226 & 1367 A 9 & 16 & 240 & 420 & 2 & \text { AFHII }\end{array}$ $\begin{array}{lllllll}227 & 157 D B 9 & 16 & 240 & 420 & 2 & \text { AFHII }\end{array}$ 228 13496E $16 \quad 240420 \quad 2$ AFHIJ 229 156DA9 162404202 AFHIJ $230 \quad 1347$ A9 $16 \quad 240 \quad 420 \quad 2$ AFIII 231 1569F4 $32 \quad 240 \quad 420 \quad 2 \quad$ BBGGH 232 13D8A4 $16 \quad 240 \quad 420 \quad 2 \quad$ BBGGJ 233 143EC9 $16240 \quad 420 \quad 2 \quad$ BBGGJ 234 143FD8 $16240 \quad 4202$ BBGGJ 235 5EFF7A $32 \quad 240 \quad 420 \quad 2 \quad$ BBGHH 236 136B3E $16 \quad 240 \quad 420 \quad 2 \quad$ BBGHH 237 136F3A $16 \quad 240 \quad 420 \quad 2 \quad$ BBGHH 238 13EB74 $16240 \quad 420 \quad 2 \quad$ BBGHH 239 13EA74 $16 \quad 240 \quad 420 \quad 2 \quad$ BBGHI 240 15FA9C $16 \quad 240 \quad 420 \quad 2 \quad$ BBGHI 241 13683D $16 \quad 240 \quad 420 \quad 2 \quad$ BBGHJ 242 136D38 $16240 \quad 420 \quad 2 \quad$ BBGHJ 243 13D874 $16 \quad 240 \quad 420 \quad 2 \quad$ BBGHJ 244 13EE74 $16 \quad 240 \quad 420 \quad 2 \quad$ BBGHJ 245 1437BD $16240 \quad 4202$ BBGHJ $24613 D 836 \quad 16240 \quad 420 \quad 2 \quad$ BBGIJ 247 13D974 $16240 \quad 420 \quad 2 \quad$ BBGIJ 248 1437CA $16 \quad 240420 \quad 2$ BBGIJ 249 1539AF $16 \quad 240 \quad 420 \quad 2$ BBGIJ 250 13DD74 $16 \quad 240 \quad 420 \quad 2 \quad$ BBGJ J 251 13EF74 $16 \quad 240 \quad 420 \quad 2 \quad$ BBHHH 252 13693C $16240420 \quad 2 \quad$ BBHHI $\begin{array}{lllllll}253 & 136 \mathrm{C} 39 & 16 & 240 & 420 & 2 & \text { BBHHI }\end{array}$ 254 13D9B5 $16 \quad 240 \quad 420 \quad 2 \quad$ BBHHI 255 13DC74 $16 \quad 240 \quad 420 \quad 2 \quad$ BBHHJ 256 156AD9 $16 \quad 240 \quad 420 \quad 2 \quad$ BBHHJ 257 153CA4 $32 \quad 240420 \quad 2 \quad$ BBHII $\begin{array}{lllllll}258 & 136 \mathrm{~A} 3 \mathrm{~F} & 16 & 240 & 420 & 2 & \mathrm{BBHIJ}\end{array}$ 259 136E3B $16 \quad 240 \quad 420 \quad 2$ BBHIJ $260143 \mathrm{C} 69 \quad 16240 \quad 420 \quad 2 \quad \mathrm{BFGGH}$ 261 147CA9 $16240 \quad 420 \quad 2 \quad$ BFGGH $\begin{array}{lllllll}262 & 14379 \mathrm{C} & 16 & 240 & 420 & 2 & \mathrm{BFGHH}\end{array}$ $\begin{array}{lllllll}263 & 14396 \mathrm{C} & 16 & 240 & 420 & 2 & \mathrm{BFGHH}\end{array}$ 264 14B7C9 $16240 \quad 4202$ BFGHI 265 1437BE 162404202 BFGHJ

TABLE 3 (continued). 


\section{$\begin{array}{lllllll}N & S P & S & H_{1} & H_{2} & H_{3} & L T\end{array}$}

266 1358EC $16240330 \quad 1$ AGGIJ

267 1368B4 $16240330 \quad 1$ AGGIJ

$\begin{array}{lllllll}268 & 13 \mathrm{E} 837 & 16 & 240 & 330 & 1 & \text { AGGIJ }\end{array}$

269 143FCA 162403301 AGGIJ

$\begin{array}{lllllll}270 & 13482 F & 16 & 240 & 330 & 1 & \text { AGGJ J }\end{array}$

271 143A9F $16 \quad 240330 \quad 1$ AGGJ J

272 13EF84 $16240330 \quad 1$ AGHHJ

273 1359FD $16 \quad 240330 \quad 1$ AGHII

274 13E936 $16240330 \quad 1$ AGHII

275 13EA94 $16240330 \quad 1$ AGHII

276 134A3C $16 \quad 240330 \quad 1$ AGHIJ

$\begin{array}{lllllll}277 & 136 \mathrm{C} 29 & 16 & 240 & 330 & 1 & \text { AGHIJ }\end{array}$

278 13EE9B $16 \quad 240 \quad 330 \quad 1$ AGHIJ

279 14AB9C $16240330 \quad 1$ AGHIJ

280 14FB9C $16 \quad 240330 \quad 1$ AGHJJ

281 136A94 $16 \quad 240 \quad 330 \quad 1$ AGIIJ

$282 \quad 13$ CA37 $16240330 \quad 1$ AGIIJ

283 13493F $16240330 \quad 1$ AGIJJ

284 134A2D $16240330 \quad 1$ AGIJJ

285 136E2B $16240330 \quad 1$ AGIJJ

286 1347EC $16240330 \quad 1$ AHHIJ

287 1349FD $16 \quad 240330 \quad 1$ AHIIJ

288 1347FD $16240 \quad 330 \quad 1$ AHIJ J

$28913678 \mathrm{C} \quad 16240330 \quad 1$ AHIJ J

290 13496D $16240330 \quad 1$ AIIJJ

291 136DA9 $16240330 \quad 1$ AIJJJ

292 1358ED $16 \quad 240 \quad 330 \quad 1 \quad$ BGGGH

293 13EB94 $16240330 \quad 1 \quad$ BGGGH

294 13EBC4 $16 \quad 240330 \quad 1 \quad$ BGGGH

295 13DB34 $16 \quad 240330 \quad 1$ BGGGJ

$29613683 \mathrm{C} \quad 16240330 \quad 1 \quad \mathrm{BGGHH}$

297 136BCE $16 \quad 240 \quad 330 \quad 1 \quad$ BGGHH

$298136 \mathrm{C} 38 \quad 16240330 \quad 1 \quad$ BGGHH

299 13EC64 $16 \quad 240 \quad 330 \quad 1 \quad$ BGGHH

300 13EF9A $16 \quad 240 \quad 330 \quad 1 \quad$ BGGHH

301 1368BE $16 \quad 240330 \quad 1$ BGGHI

302 136F9A $16240330 \quad 1$ BGGHI

303 13C9B4 $16240330 \quad 1$ BGGHI

304 13CB74 $16240 \quad 330 \quad 1 \quad$ BGGHI

$\begin{array}{lllllll}305 & 13 D 837 & 16 & 240 & 330 & 1 & \text { BGGHI }\end{array}$

306 13DA35 $16 \quad 240330 \quad 1$ BGGHI

307 13E964 $16 \quad 240330 \quad 1 \quad$ BGGHI

$\begin{array}{lllllll}308 & 1569 B F & 16 & 240 & 330 & 1 & \text { BGGHI }\end{array}$

309 13587D $16 \quad 240 \quad 330 \quad 1 \quad$ BGGHJ

$\begin{array}{lllllll}310 & 135 B 2 D & 16 & 240 & 330 & 1 & \text { BGGHJ }\end{array}$

311 135B3D $16 \quad 240 \quad 330 \quad 1 \quad$ BGGHJ

312 135B7F $16 \quad 240 \quad 330 \quad 1 \quad$ BGGHJ

$\begin{array}{lllllll}313 & 13683 \mathrm{E} & 16 & 240 & 330 & 1 & \text { BGGHJ }\end{array}$

314 136B2D $16 \quad 240330 \quad 1 \quad$ BGGHJ

315 136B3F $16 \quad 240 \quad 330 \quad 1 \quad$ BGGHJ

316 136B9C $16240 \quad 330 \quad 1 \quad$ BGGHJ

$\begin{array}{lllllll}317 & 136 \mathrm{C} 3 \mathrm{~A} & 16 & 240 & 330 & 1 & \text { BGGHJ }\end{array}$

318 136E3A $16240330 \quad 1$ BGGHJ

319 13EEDA $16240330 \quad 1$ BGGHJ

320 1569EA 162403301 BGGHJ
$\begin{array}{lllllll}N & S P & S & H_{1} & H_{2} & H_{3} & L T\end{array}$

321 156FC9 $16 \quad 240 \quad 330 \quad 1 \quad$ BGGHJ

$\begin{array}{lllllll}322 & 1357 A 8 & 16 & 240 & 330 & 1 & \text { BGGIJ }\end{array}$

$\begin{array}{lllllll}323 & 1359 F C & 16 & 240 & 330 & 1 & \text { BGGIJ }\end{array}$

$\begin{array}{lllllll}324 & 1368 E 4 & 16 & 240 & 330 & 1 & \text { BGGIJ }\end{array}$

325 136DB8 $16 \quad 240330 \quad 1$ BGGIJ

$\begin{array}{lllllll}326 & 13 C 7 A 8 & 16 & 240 & 330 & 1 & \text { BGGIJ }\end{array}$

$\begin{array}{lllllll}327 & 13 \text { CA35 } 16 & 240 & 330 & 1 & \text { BGGIJ }\end{array}$

328 1539BF $16 \quad 240330 \quad 1 \quad$ BGGIJ

$\begin{array}{lllllll}329 & 13582 \mathrm{E} & 16 & 240 & 330 & 1 & \text { BGGJJ }\end{array}$

$\begin{array}{lllllll}330 & 13583 E & 16 & 240 & 330 & 1 & \text { BGGJJ }\end{array}$

331 13587C $16 \quad 240 \quad 330 \quad 1 \quad$ BGGJJ

332 135B7E $16 \quad 240330 \quad 1$ BGGJJ

$\begin{array}{lllllll}333 & 136 B 3 D & 16 & 240 & 330 & 1 & \text { BGGJ J }\end{array}$

334 136E38 $16 \quad 240 \quad 330 \quad 1 \quad$ BGGJJ

335 1439BE $16 \quad 240330 \quad 1 \quad$ BGGJJ

$\begin{array}{lllllll}336 & 143 A 69 & 16 & 240 & 330 & 1 & \text { BGGJJ }\end{array}$

337 1579ED $16 \quad 240330 \quad 1 \quad$ BGGJJ

$\begin{array}{lllllll}338 & 1369 E C & 16 & 240 & 330 & 1 & \text { BGHHI }\end{array}$

$\begin{array}{lllllll}339 & 136 \mathrm{~A} 3 \mathrm{E} & 16 & 240 & 330 & 1 & \mathrm{BGHHI}\end{array}$

$\begin{array}{lllllll}340 & 136 \mathrm{CB} 9 & 16 & 240 & 330 & 1 & \text { BGHHI }\end{array}$

$\begin{array}{lllllll}341 & 136 F 29 & 16 & 240 & 330 & 1 & \text { BGHHI }\end{array}$

342 136F 3B $16 \quad 240330 \quad 1 \quad$ BGHHI

$\begin{array}{lllllll}343 & 13 C 974 & 16 & 240 & 330 & 1 & \text { BGHHI }\end{array}$

344 13CCE6 $16 \quad 240 \quad 330 \quad 1 \quad$ BGHHI

345 13EFD4 $16 \quad 240 \quad 330 \quad 1 \quad$ BGHHI

$\begin{array}{lllllll}346 & 357 B 9 D & 16 & 240 & 330 & 1 & \text { BGHHI }\end{array}$

347 13CCE5 $16 \quad 240 \quad 330 \quad 1 \quad$ BGHHJ

348 13DC64 $16 \quad 240 \quad 330 \quad 1 \quad$ BGHHJ

$\begin{array}{lllllll}349 & 1479 \mathrm{EC} & 16 & 240 & 330 & 1 & \text { BGHHJ }\end{array}$

$350 \quad 157 A 3 C \quad 16 \quad 240 \quad 330 \quad 1 \quad$ BGHHJ

351 15EA9C $16 \quad 240330 \quad 1$ BGHHJ

$\begin{array}{lllllll}352 & 136 \mathrm{~A} 9 \mathrm{C} & 16 & 240 & 330 & 1 & \text { BGHII }\end{array}$

$\begin{array}{lllllll}353 & 13 \text { C936 } & 16 & 240 & 330 & 1 & \text { BGHII }\end{array}$

354 15FB9D $16 \quad 240 \quad 330 \quad 1$ BGHII

$\begin{array}{lllllll}355 & 13592 F & 16 & 240 & 330 & 1 & \text { BGHIJ }\end{array}$

$\begin{array}{lllllll}356 & 13593 \mathrm{~F} & 16 & 240 & 330 & 1 & \text { BGHIJ }\end{array}$

$\begin{array}{lllllll}357 & 13597 \mathrm{D} & 16 & 240 & 330 & 1 & \text { BGHIJ }\end{array}$

$\begin{array}{lllllll}358 & 135 \mathrm{~A} 7 \mathrm{~F} & 16 & 240 & 330 & 1 & \text { BGHIJ }\end{array}$

$\begin{array}{lllllll}359 & 13692 \mathrm{~F} & 16 & 240 & 330 & 1 & \text { BGHIJ }\end{array}$

$\begin{array}{lllllll}360 & 13693 D & 16 & 240 & 330 & 1 & \text { BGHIJ }\end{array}$

$\begin{array}{lllllll}361 & 1369 B E & 16 & 240 & 330 & 1 & \text { BGHIJ }\end{array}$

362 136A3C $16 \quad 240330 \quad 1 \quad$ BGHIJ

$\begin{array}{lllllll}363 & 136 D 2 B & 16 & 240 & 330 & 1 & \text { BGHIJ }\end{array}$

$\begin{array}{lllllll}364 & 136 \mathrm{D} 39 & 16 & 240 & 330 & 1 & \text { BGHIJ }\end{array}$

$\begin{array}{lllllll}365 & 136 \mathrm{E} 9 \mathrm{~B} & 16 & 240 & 330 & 1 & \text { BGHIJ }\end{array}$

$\begin{array}{lllllll}366 & 136 F 39 & 16 & 240 & 330 & 1 & \text { BGHIJ }\end{array}$

$\begin{array}{lllllll}367 & 13 D 964 & 16 & 240 & 330 & 1 & \text { BGHIJ }\end{array}$

368 13DCE4 $16 \quad 240 \quad 330 \quad 1 \quad$ BGHIJ

369 13EAC4 $16 \quad 240 \quad 330 \quad 1 \quad$ BGHIJ

$\begin{array}{lllllll}370 & 13 E B 75 & 16 & 240 & 330 & 1 & \text { BGHIJ }\end{array}$

371 13EEC6 $16 \quad 240330 \quad 1$ BGHIJ

$\begin{array}{lllllll}372 & 13 E E C B & 16 & 240 & 330 & 1 & \text { BGHIJ }\end{array}$

373 14A93C $16 \quad 240330 \quad 1$ BGHIJ

$\begin{array}{lllllll}374 & 15396 \mathrm{D} & 16 & 240 & 330 & 1 & \text { BGHIJ }\end{array}$

375 1569FD $16 \quad 240330 \quad 1$ BGHIJ
$\begin{array}{lllllll}N & S P & S & H_{1} & H_{2} & H_{3} & L T\end{array}$

$376 \quad$ 13EEC5 $16 \quad 240 \quad 330 \quad 1 \quad$ BGHJJ

$\begin{array}{lllllll}377 & 13 E E D 5 & 16 & 240 & 330 & 1 & \text { BGHJJ }\end{array}$

$\begin{array}{lllllll}378 & 156 \mathrm{E} 9 \mathrm{D} & 16 & 240 & 330 & 1 & \text { BGHJJ }\end{array}$

$\begin{array}{lllllll}379 & 1357 B 9 & 16 & 240 & 330 & 1 & \text { BGIIJ }\end{array}$

$\begin{array}{lllllll}380 & 136 \mathrm{AC} 4 & 16 & 240 & 330 & 1 & \text { BGIIJ }\end{array}$

$\begin{array}{lllllll}381 & 13 D 936 & 16 & 240 & 330 & 1 & \text { BGIIJ }\end{array}$

$\begin{array}{lllllll}382 & 15396 \mathrm{~A} & 16 & 240 & 330 & 1 & \text { BGIIJ }\end{array}$

$\begin{array}{lllllll}383 & 13597 \mathrm{C} & 16 & 240 & 330 & 1 & \text { BGIJJ }\end{array}$

$\begin{array}{lllllll}384 & 135 \mathrm{~A} 2 \mathrm{C} & 16 & 240 & 330 & 1 & \mathrm{BGIJJ}\end{array}$

$\begin{array}{lllllll}385 & 135 A 3 C & 16 & 240 & 330 & 1 & \text { BGIJJ }\end{array}$

$\begin{array}{lllllll}386 & 135 \mathrm{~A} 7 \mathrm{E} & 16 & 240 & 330 & 1 & \mathrm{BGIJJ}\end{array}$

$\begin{array}{lllllll}387 & 13693 \mathrm{~F} & 16 & 240 & 330 & 1 & \mathrm{BGIJJ}\end{array}$

$\begin{array}{llllllll}388 & 136 \mathrm{D} 3 \mathrm{~B} & 16 & 240 & 330 & 1 & \text { BGIJJ }\end{array}$

389 13D9B4 $16 \quad 240 \quad 330 \quad 1 \quad$ BGIJJ

$\begin{array}{lllllll}390 & 14396 \mathrm{~A} & 16 & 240 & 330 & 1 & \text { BGIJJ }\end{array}$

$\begin{array}{lllllll}391 & 13 \mathrm{D} 9 \mathrm{~A} 5 & 16 & 240 & 330 & 1 & \text { BHHHI }\end{array}$

$\begin{array}{lllllll}392 & 13 D C 65 & 16 & 240 & 330 & 1 & \text { BHHHI }\end{array}$

$\begin{array}{lllllll}393 & 13 D C 75 & 16 & 240 & 330 & 1 & \text { BHHHI }\end{array}$

394 156A3E $16 \quad 240 \quad 330 \quad 1 \quad$ BHHHJ

$\begin{array}{lllllll}395 & 13 D 965 & 16 & 240 & 330 & 1 & \text { BHHII }\end{array}$

$\begin{array}{lllllll}396 & 13 E F 75 & 16 & 240 & 330 & 1 & \text { BHHIJ }\end{array}$

$\begin{array}{lllllll}397 & \text { 13EFD5 } & 16 & 240 & 330 & 1 & \text { BHHIJ }\end{array}$

$\begin{array}{lllllll}398 & 156 B 3 D & 16 & 240 & 330 & 1 & \text { BHHIJ }\end{array}$

$\begin{array}{llllllll}399 & 156 D 39 & 16 & 240 & 330 & 1 & \text { BHHIJ }\end{array}$

$400 \quad$ 143CE9 $16 \quad 240 \quad 330 \quad 1 \quad$ FGGGH

401 136B94 $16 \quad 240 \begin{array}{lllll}330 & 1 & \text { FGGGI }\end{array}$

$402 \quad 136 B C 4 \quad 16 \quad 240 \quad 330 \quad 1 \quad$ FGGGI

$403 \quad 143 F 9 C \quad 16 \quad 240 \quad 330 \quad 1 \quad$ FGGHH

$\begin{array}{lllllll}404 & 13679 \mathrm{~A} & 16 & 240 & 330 & 1 & \text { FGGHI }\end{array}$

$\begin{array}{llllllll}405 & 1367 \mathrm{~B} 8 & 16 & 240 & 330 & 1 & \text { FGGHI }\end{array}$

$406 \quad$ 14BAC9 $16 \quad 240 \quad 330 \quad 1 \quad$ FGGHI

407 14B93C $16 \quad 240 \begin{array}{lllll}330 & 1 & \text { FGGHJ }\end{array}$

$\begin{array}{lllllll}408 & 15397 D & 16 & 240 & 330 & 1 & \text { FGGHJ }\end{array}$

409 153B9F $16 \quad 240 \quad 330 \quad 1 \quad$ FGGHJ

$\begin{array}{lllllll}410 & 1569 E D & 16 & 240 & 330 & 1 & \text { FGGHJ }\end{array}$

411 1369B4 $16 \quad 240 \quad 330 \quad 1 \quad$ FGGII

$\begin{array}{lllllll}412 & 1369 E 4 & 16 & 240 & 330 & 1 & \text { FGGII }\end{array}$

$\begin{array}{lllllll}413 & 13 C 7 B 8 & 16 & 240 & 330 & 1 & \text { FGGII }\end{array}$

$\begin{array}{lllllll}414 & 1357 B 8 & 16 & 240 & 330 & 1 & \text { FGGIJ }\end{array}$

$\begin{array}{lllllll}415 & 156 \mathrm{AC} 9 & 16 & 240 & 330 & 1 & \text { FGHHI }\end{array}$

$\begin{array}{lllllll}416 & 157 D 39 & 16 & 240 & 330 & 1 & \text { FGHHI }\end{array}$

$\begin{array}{lllllll}417 & 157 \mathrm{~A} 3 \mathrm{E} & 16 & 240 & 330 & 1 & \mathrm{FGHHJ}\end{array}$

$418 \quad 157 \mathrm{~F} 9 \mathrm{C} \quad 16 \quad 240 \quad 330 \quad 1 \quad$ FGHJJ

$\begin{array}{lllllll}419 & 1357 A 9 & 16 & 240 & 330 & 1 & \text { FGIIJ }\end{array}$

$420 \quad 13 E B 85 \quad 16 \quad 240 \quad 321 \quad 1 \quad$ AGHIJ

421 153B8F $16 \quad 240 \quad 321 \quad 1 \quad$ AHHJJ

$422 \quad 157 \mathrm{~F} 8 \mathrm{C} \quad 16 \quad 240 \quad 321 \quad 1 \quad$ AHHJ

$423 \quad 1367 \mathrm{AE} \quad 16 \quad 240 \quad 321 \quad 1 \quad$ AHIJJ

424 13EFDA $16 \quad 240 \quad 321 \quad 1 \quad$ BGHHI

$\begin{array}{lllllll}425 & 13 E F D B & 16 & 240 & 321 & 1 & \text { BGHII }\end{array}$

426 146F28 $32 \quad 240240 \quad 0 \quad$ GGGGH

427 13FFC8 $32 \quad 240240 \quad 0 \quad$ GGGHH

$\begin{array}{lllllll}428 & 13 \mathrm{C} 8 \mathrm{~F} 4 & 16 & 240 & 240 & 0 & \mathrm{GGGHI}\end{array}$

$429 \quad 13583 F \quad 16 \quad 240 \quad 240 \quad 0 \quad$ GGGHJ

$\begin{array}{lllllll}430 & 13 E 935 & 16 & 240 & 240 & 0 & \text { GGGIJ }\end{array}$

TABLE 3 (continued). 


\section{$\begin{array}{lllllll}N & S P & S & H_{1} & H_{2} & H_{3} & L T\end{array}$}

431 13EE6A $16240240 \quad 0 \quad$ GGGIJ 432 135B3C $16240240 \quad 0 \quad$ GGGJJ 433 13EE9A $16240240 \quad 0 \quad$ GGGJJ 434 136FC8 $32240240 \quad 0 \quad$ GGHHH 435 1358FD $16240240 \quad 0 \quad$ GGHHH $\begin{array}{lllllll}436 & 13586 \mathrm{D} & 16 & 240 & 240 & 0 & \mathrm{GGHHI}\end{array}$ $\begin{array}{lllllll}437 & 1368 \mathrm{EC} & 16 & 240 & 240 & 0 & \mathrm{GGHHI}\end{array}$ $\begin{array}{lllllll}438 & 136 \mathrm{C} 2 \mathrm{~B} & 16 & 240 & 240 & 0 & \mathrm{GGHHI}\end{array}$ 439 136DEA $16240240 \quad 0$ GGHHJ 440 13ED64 $16240240 \quad 0$ GGHHJ 441 157DA4 $32240240 \quad 0$ GGHII $442 \quad 157 \mathrm{DF} 4 \quad 32 \quad 240240 \quad 0 \quad$ GGHII 443 135A3D $16240240 \quad 0 \quad$ GGHIJ 444 135B6E $16240240 \quad 0$ GGHIJ $445 \quad 136 \mathrm{E} 29 \quad 16 \quad 240 \quad 240 \quad 0 \quad$ GGHIJ 446 136F2B $16 \quad 240240 \quad 0$ GGHIJ 447 13CB75 $16240240 \quad 0$ GGHIJ $448 \quad 13 C C 6516240240 \quad 0 \quad$ GGHIJ 449 1539EA $16240240 \quad 0$ GGHIJ 450 136CB8 $16240240 \quad 0$ GGHJJ 451 13EB95 $16 \quad 240240 \quad 0$ GGHJJ 452 157E9D $16240240 \quad 0$ GGHJJ $\begin{array}{llllllll}453 & 13 C 937 & 16 & 240 & 240 & 0 & \text { GGIIJ }\end{array}$ 454 13593E $16240240 \quad 0 \quad$ GGIJJ 455 136B9D $16240240 \quad 0 \quad$ GGIJJ $456 \quad 136 \mathrm{D} 2916240240 \quad 0 \quad$ GGIJJ 457 13EE65 $16240240 \quad 0 \quad$ GGIJJ 458 136E9A $16240240 \quad 0$ GGJJJ 459 13EFC6 $16 \quad 240 \quad 240 \quad 0$ GHHHI 460 5BFFDA $32 \quad 240240 \quad 0$ GHHII 461 135A6F $16 \quad 240240 \quad 0$ GHHII 462 136ACE $16240240 \quad 0$ GHHII 463 13EFCB $16240240 \quad 0$ GHHII 464 156CEA $16240240 \quad 0$ GHHII 465 1359EC $16240240 \quad 0$ GHHIJ 466 13CDE6 $16240240 \quad 0$ GHHIJ 467 13EF65 $16240240 \quad 0$ GHHIJ 468 13EFC5 $16240240 \quad 0$ GHHIJ 469 156ACE $16240240 \quad 0$ GHHIJ 470 15EB9D $16240240 \quad 0$ GHHIJ $\begin{array}{lllllll}471 & 357 \mathrm{AB} 9 & 16 & 240 & 240 & 0 & \text { GHHIJ }\end{array}$ 472 153A9E $16240240 \quad 0$ GHHJ $473 \quad 13596 \mathrm{C} \quad 16240240 \quad 0$ GHIIJ $474 \quad 13 C 975 \quad 16 \quad 240240 \quad 0 \quad$ GHIIJ 475 156DB9 $16240240 \quad 0$ GHIIJ 476 13C9B5 $16240240 \quad 0$ GHIJ J 477 13CD65 $16240240 \quad 0$ GHIJ J $478 \quad 13 C D 75 \quad 16 \quad 240240 \quad 0$ GHIJJ 479 157DA9 $16240240 \quad 0$ GHIJJ 480 1369BF $16240240 \quad 0$ GIIJJ 481 367B9C $32240240 \quad 0$ HHHJ J $482 \quad 13582 \mathrm{~F} \quad 16 \quad 240231 \quad 0 \quad$ GGGHJ $483 \quad 13682 \mathrm{~F} \quad 16 \quad 240231 \quad 0 \quad$ GGGHJ 484 135B2C $16240231 \quad 0 \quad$ GGGJJ 485 135B6F $16240231 \quad 0 \quad$ GGHHI
$\begin{array}{lllllll}N & S P & S & H_{1} & H_{2} & H_{3} & L T\end{array}$

486 1368BF $16240231 \quad 0$ GGHHJ 487 13EDA8 $16 \quad 240 \quad 231 \quad 0 \quad$ GGHHJ 488 13EF6A $16240231 \quad 0 \quad$ GGHHJ 489367 AB5 $16240231 \quad 0 \quad$ GGHII 490 13586C $16240231 \quad 0 \quad$ GGHIJ 491 135A2D $16240231 \quad 0 \quad$ GGHIJ 492 136A2D $16 \quad 240231 \quad 0 \quad$ GGHIJ 493 136CEA $16240231 \quad 0$ GGHIJ $494 \quad 13 C 86516240231 \quad 0 \quad$ GGHIJ 495 1358FC $16240231 \quad 0 \quad$ GGHJJ 496 13C8F5 $16 \quad 240231 \quad 0 \quad$ GGHJJ 497 13592E $16240231 \quad 0 \quad$ GGIJJ $\begin{array}{lllllll}498 & 1367 C 8 & 16 & 240 & 231 & 0 & \text { GGIJJ }\end{array}$ 499 1367EA $16240231 \quad 0 \quad$ GGIJJ 500 13E9A6 $16 \quad 240231 \quad 0$ GHHHI $\begin{array}{lllllll}501 & 13596 \mathrm{D} & 16 & 240 & 231 & 0 & \text { GHHII }\end{array}$ 502 136F9B $16240231 \quad 0$ GHHII 503 13EF9B $16240231 \quad 0$ GHHII 504 1359ED $16240231 \quad 0$ GHHIJ 505 136A9D $16240231 \quad 0$ GHHIJ 506 13EA95 $16 \quad 240231 \quad 0 \quad$ GHHIJ 507 135A6E $16240231 \quad 0$ GHIIJ $\begin{array}{lllllll}508 & 13679 B & 16 & 240 & 231 & 0 & \text { GHIIJ }\end{array}$ 509 1367B9 $16 \quad 240231 \quad 0 \quad$ GHIIJ $\begin{array}{lllllll}510 & 136 D B 9 & 16 & 240 & 231 & 0 & \text { GHIIJ }\end{array}$ 511 13C7B9 $16240231 \quad 0 \quad$ GHIIJ 512 13C9A5 $16240231 \quad 0$ GHIIJ $\begin{array}{lllllll}513 & 13 E A 65 & 16 & 240 & 231 & 0 & \text { GHIIJ }\end{array}$ 514 357BAF $32 \quad 240231 \quad 0 \quad$ HHHII $\begin{array}{lllllll}515 & 357 \mathrm{~B} 9 \mathrm{~A} & 16 & 240 & 231 & 0 & \text { HHHII }\end{array}$ $\begin{array}{lllllll}516 & 367 B 95 & 16 & 240 & 231 & 0 & \text { HHHIJ }\end{array}$ 517 1569AF $32 \quad 231510 \quad 3$ ABBGG 518 1579BF $32 \quad 231 \quad 420 \quad 2$ AAGII 519 1347AC $16 \quad 231420 \quad 2$ ABHIJ $520 \quad 134 \mathrm{~A} 6 \mathrm{~F} \quad 16 \quad 231 \quad 420 \quad 2 \quad$ ABHIJ 521 1369AF $16 \quad 231420 \quad 2$ ABIIJ $\begin{array}{lllllll}522 & 1347 \mathrm{BD} & 16 & 231 & 420 & 2 & \text { ABIJJ }\end{array}$ $\begin{array}{lllllll}523 & 1539 F A & 16 & 231 & 420 & 2 & \text { AFGGI }\end{array}$ $\begin{array}{lllllll}524 & 1479 F C & 16 & 231 & 420 & 2 & \text { BFGGH }\end{array}$ $525 \quad$ 13E7A8 $16 \quad 231330 \quad 1 \quad$ AGGIJ $\begin{array}{lllllll}526 & 13682 \mathrm{D} & 16 & 231 & 330 & 1 & \text { AGGJJ }\end{array}$ 527 136FC4 $16 \quad 231330 \quad 1$ AGHIJ 528 13E9AC $16 \quad 231330 \quad 1$ AGIIJ 529 1349ED $16 \quad 231330 \quad 1$ AGIJJ $\begin{array}{lllllll}530 & 136 \mathrm{~A} 2 \mathrm{~F} & 16 & 231 & 330 & 1 & \text { AGIJJ }\end{array}$ $\begin{array}{lllllll}531 & 136 \mathrm{DE} 4 & 16 & 231 & 330 & 1 & \text { AGIJJ }\end{array}$ $\begin{array}{lllllll}532 & 13 \mathrm{C} 8 \mathrm{~A} 5 & 16 & 231 & 330 & 1 & \mathrm{BGGHJ}\end{array}$ $\begin{array}{lllllll}533 & 13 E 8 A C & 16 & 231 & 330 & 1 & \text { FGGGI }\end{array}$ $\begin{array}{lllllll}534 & 1579 F D & 16 & 231 & 330 & 1 & \text { FGGGI }\end{array}$ $\begin{array}{lllllll}535 & 136 \mathrm{CE} 4 & 16 & 231 & 330 & 1 & \text { FGGHI }\end{array}$ $\begin{array}{lllllll}536 & 136 E C 4 & 16 & 231 & 330 & 1 & \text { FGGIJ }\end{array}$ $\begin{array}{lllllll}537 & 15397 A & 16 & 231 & 330 & 1 & \text { FGGIJ }\end{array}$ $\begin{array}{lllllll}538 & 13 E B 65 & 16 & 231 & 231 & 0 & \text { GGGIJ }\end{array}$ $\begin{array}{lllllll}539 & 136 B 2 F & 16 & 231 & 231 & 0 & \text { GGGJJ }\end{array}$ $\begin{array}{lllllll}540 & 13 C 965 & 16 & 231 & 231 & 0 & \text { GGIIJ }\end{array}$

\section{$\begin{array}{lllllll}N & S P & S & H_{1} & H_{2} & H_{3} & L T\end{array}$}

541 13692D $16231231 \quad 0 \quad$ GGIJJ $\begin{array}{lllllll}542 & 1367 C E & 16 & 231 & 231 & 0 & \text { GHHIJ }\end{array}$ $543 \quad 1367 E C \quad 16231231 \quad 0 \quad$ GHHIJ 544 357ABE $16231231 \quad 0$ GHHIJ $545 \quad 367 \mathrm{ABF} \quad 32 \quad 222 \quad 321 \quad 1 \quad$ BGGGG $\begin{array}{lllllll}546 & 13 E 7 A C & 16 & 160 & 330 & 2 & \text { BBGII }\end{array}$ 547 143DFA $16 \quad 150410 \quad 3 \quad$ ABBIJ 548 143EDB $16 \quad 150410 \quad 3$ ABBIJ $549 \quad 1477 \mathrm{AF} \quad 16 \quad 150 \quad 410 \quad 3 \quad$ ABFGH $\begin{array}{lllllll}550 & 14 \mathrm{~A} 79 \mathrm{C} & 16 & 150 & 410 & 3 & \mathrm{ABFGI}\end{array}$ $\begin{array}{lllllll}551 & 14 A 78 D & 16 & 150 & 410 & 3 & \text { ABFHH }\end{array}$ $\begin{array}{lllllll}552 & 143 B 6 E & 16 & 150 & 410 & 3 & \text { ABFHJ }\end{array}$ $\begin{array}{llllllll}553 & 14386 \mathrm{~F} & 16 & 150 & 410 & 3 & \mathrm{ABFJJ}\end{array}$ 554 14F7CA $16 \quad 150410 \quad 3 \quad$ BBBGJ $\begin{array}{lllllll}555 & 1437 \mathrm{AC} & 16 & 150 & 410 & 3 & \text { BBBHJ }\end{array}$ $\begin{array}{lllllll}556 & 1437 \mathrm{DB} & 16 & 150 & 410 & 3 & \text { BBBIJ }\end{array}$ $\begin{array}{lllllll}557 & 14279 F & 16 & 150 & 410 & 3 & \text { BBFGG }\end{array}$ $\begin{array}{lllllll}558 & 1427 \mathrm{AF} & 16 & 150 & 410 & 3 & \mathrm{BBFGG}\end{array}$ $\begin{array}{lllllll}559 & 14779 A & 16 & 150 & 410 & 3 & \text { BBFGH }\end{array}$ $\begin{array}{lllllll}560 & 1477 \mathrm{~A} 9 & 16 & 150 & 410 & 3 & \mathrm{BBFGH}\end{array}$ $\begin{array}{lllllll}561 & 14 A 7 C 9 & 16 & 150 & 410 & 3 & \text { BBFGI }\end{array}$ $\begin{array}{lllllll}562 & 143 E 69 & 16 & 150 & 410 & 3 & \text { BBFGJ }\end{array}$ $\begin{array}{lllllll}563 & 14 F 79 \mathrm{C} & 16 & 150 & 410 & 3 & \text { BBFGJ }\end{array}$ $\begin{array}{lllllll}564 & 143 \mathrm{E} 6 \mathrm{~B} & 16 & 150 & 410 & 3 & \mathrm{BBFHI}\end{array}$ $\begin{array}{lllllll}565 & 1437 \mathrm{AF} & 16 & 150 & 410 & 3 & \mathrm{BBFHJ}\end{array}$ $\begin{array}{lllllll}566 & 143 \mathrm{D} 6 \mathrm{~A} & 16 & 150 & 410 & 3 & \mathrm{BBFIJ}\end{array}$ $\begin{array}{lllllll}567 & 1477 \mathrm{~A} 8 & 16 & 150 & 320 & 2 & \text { ABGGJ }\end{array}$ $\begin{array}{lllllll}568 & \text { 153CFA } & 16 & 150 & 320 & 2 & \text { ABIII }\end{array}$ 569 157CEA $16 \quad 150320 \quad 2$ ABIII $\begin{array}{llllll}570 & 14 A B 3 C & 16 & 150 & 320 & 2\end{array}$ ABIJJ $\begin{array}{lllllll}571 & 13 E E 85 & 16 & 150 & 320 & 2 & \text { ABJJJ }\end{array}$ $\begin{array}{lllllll}572 & 14 A B 78 & 16 & 150 & 320 & 2 & A F G G J\end{array}$ $\begin{array}{lllllll}573 & 134 \mathrm{~A} 7 \mathrm{C} & 16 & 150 & 320 & 2 & \mathrm{AFHIJ}\end{array}$ $\begin{array}{lllllll}574 & 15 B A 79 & 16 & 150 & 320 & 2 & \text { AFHIJ }\end{array}$ $\begin{array}{lllllll}575 & 15 A D 69 & 16 & 150 & 320 & 2 & \text { AFIII }\end{array}$ $\begin{array}{lllllll}576 & 3579 \mathrm{CF} & 16 & 150 & 320 & 2 & \text { AFIII }\end{array}$ $\begin{array}{lllllll}577 & 1347 \mathrm{ED} & 16 & 150 & 320 & 2 & \text { AFIIJ }\end{array}$ $\begin{array}{lllllll}578 & 13497 \mathrm{~F} & 16 & 150 & 320 & 2 & \text { AFIJJ }\end{array}$ $\begin{array}{lllllll}579 & 13678 D & 16 & 150 & 320 & 2 & \text { AFIJJ }\end{array}$ $\begin{array}{lllllll}580 & 1367 \mathrm{AF} & 16 & 150 & 320 & 2 & \mathrm{AFIJJ}\end{array}$ 581 15AD6E $16 \quad 150320 \quad 2 \quad$ AFIJJ $\begin{array}{lllllll}582 & 13 D 8 F 4 & 16 & 150 & 320 & 2 & \text { BBGGJ }\end{array}$ $\begin{array}{lllllll}583 & \text { 13EED8 } & 16 & 150 & 320 & 2 & \text { BBGGJ }\end{array}$ $\begin{array}{lllllll}584 & 1437 \mathrm{E} 8 & 16 & 150 & 320 & 2 & \mathrm{BBGGJ}\end{array}$ $\begin{array}{lllllll}585 & 14779 B & 16 & 150 & 320 & 2 & \text { BBGGJ }\end{array}$ $\begin{array}{lllllll}586 & \text { 13EBD4 } & 16 & 150 & 320 & 2 & \text { BBGHH }\end{array}$ $\begin{array}{lllllll}587 & 136 \mathrm{BDE} & 16 & 150 & 320 & 2 & \mathrm{BBGHI}\end{array}$ $\begin{array}{lllllll}588 & 136 \mathrm{CFA} & 16 & 150 & 320 & 2 & \mathrm{BBGHI}\end{array}$ $\begin{array}{lllllll}589 & 13 D B 75 & 16 & 150 & 320 & 2 & \text { BBGHI }\end{array}$ $\begin{array}{lllllll}590 & 156 \mathrm{CFA} & 16 & 150 & 320 & 2 & \mathrm{BBGHI}\end{array}$ $\begin{array}{lllllll}591 & 1368 F D & 16 & 150 & 320 & 2 & \text { BBGHJ }\end{array}$ 592 136FD8 $16 \quad 150 \quad 320 \quad 2 \quad$ BBGHJ 593 13D8E4 $16 \quad 150 \quad 320 \quad 2 \quad$ BBGHJ 594 13DDE7 $16 \quad 150 \quad 320 \quad 2 \quad$ BBGHJ $595 \quad 13 E 874 \quad 16 \quad 150320 \quad 2 \quad$ BBGHJ

TABLE 3 (continued). 


\section{$\begin{array}{lllllll}N & S P & S & H_{1} & H_{2} & H_{3} & L T\end{array}$}

596 13E8B7 $16 \quad 150320 \quad 2 \quad$ BBGHJ 597 13EF7A $16 \quad 150320 \quad 2$ BBGHJ $598 \quad 1477 \mathrm{DF} \quad 16 \quad 150320 \quad 2 \quad \mathrm{BBGHJ}$ 599 1477EC $16 \quad 150320 \quad 2 \quad$ BBGHJ 600 147CEB $16 \quad 150320 \quad 2$ BBGHJ 601 136BD4 $16 \quad 150320 \quad 2 \quad$ BBGI J 602 136ED8 $16 \quad 150320 \quad 2 \quad$ BBGIJ 603 13DA64 $16 \quad 150320 \quad 2 \quad$ BBGIJ 604 13E875 $16 \quad 150320 \quad 2 \quad$ BBGIJ 605 13EDB8 $16 \quad 1503202$ BBGIJ 606 13EE7B $16 \quad 150320 \quad 2 \quad$ BBGI J 607 14BEC9 $16 \quad 150320 \quad 2$ BBGIJ 608 156CBF $16 \quad 150320 \quad 2 \quad$ BBGI J 609 136DFA $16 \quad 150320 \quad 2 \quad$ BBGJJ $610 \quad 13 D B 74 \quad 16 \quad 150 \quad 320 \quad 2 \quad$ BBGJ J 611 13DDA4 $16 \quad 150320 \quad 2 \quad$ BBGJ J 612 13EE7A $16 \quad 150320 \quad 2 \quad$ BBGJ J 613 13EED7 $16 \quad 150320 \quad 2 \quad$ BBGJJ $\begin{array}{lllllll}614 & 14379 F & 16 & 150 & 320 & 2 & \text { BBGJ J }\end{array}$ 615 13DCF7 $16 \quad 150 \quad 320 \quad 2 \quad$ BBHHI $616 \quad 13 E C B 9 \quad 16 \quad 150 \quad 320 \quad 2 \quad$ BBHHI $617 \quad 13 E C 74 \quad 16 \quad 150 \quad 320 \quad 2 \quad$ BBHHJ 618 1369FC $16 \quad 150320 \quad 2 \quad$ BBHII 619 13E974 $16 \quad 150320 \quad 2 \quad$ BBHII 620 13E9B6 $16 \quad 150 \quad 320 \quad 2 \quad$ BBHII 621 156DFB $16 \quad 150320 \quad 2 \quad$ BBHII $622136 \mathrm{ADF} \quad 16 \quad 150320 \quad 2 \quad$ BBHIJ 623 13D9F5 $16 \quad 150320 \quad 2 \quad$ BBHIJ $624 \quad 13 E C 75 \quad 16 \quad 150 \quad 320 \quad 2 \quad$ BBHIJ $625 \quad 13 E D 74 \quad 16 \quad 150 \quad 320 \quad 2 \quad$ BBHIJ $\begin{array}{lllllll}626 & 156 \mathrm{BDF} & 16 & 150 & 320 & 2 & \mathrm{BBHIJ}\end{array}$ 627 157DEB $16 \quad 150320 \quad 2 \quad$ BBHIJ $628 \quad 357 \mathrm{~F} 9 \mathrm{~B} \quad 16 \quad 150 \quad 320 \quad 2 \quad$ BBHIJ 629 13DCA4 $16 \quad 150320 \quad 2 \quad$ BBHJJ 630 13DDF7 $16 \quad 150 \quad 320 \quad 2 \quad$ BBHJJ 631 143E6D $16 \quad 150320 \quad 2 \quad$ BBHJJ 632 156F3B $16 \quad 150320 \quad 2 \quad$ BBHJJ 633 1369F4 $16 \quad 150 \quad 320 \quad 2 \quad$ BBIIJ 634 13D7B9 $16 \quad 150320 \quad 2 \quad$ BBIIJ 635 13DA74 $16 \quad 150320 \quad 2 \quad$ BBIIJ $\begin{array}{lllllll}636 & 156 \mathrm{D} 3 \mathrm{~B} & 16 & 150 & 320 & 2 & \mathrm{BBIIJ}\end{array}$ 637 13D9F4 $16 \quad 150320 \quad 2 \quad$ BBIJJ 638 13DDF6 $16 \quad 150320 \quad 2 \quad$ BBIJJ 639 143D6E $16 \quad 150320 \quad 2 \quad$ BBJJJ 640 147CAF $16 \quad 150320 \quad 2 \quad$ BFGGH $\begin{array}{lllllll}641 & 1357 \mathrm{E} 8 & 16 & 150 & 320 & 2 & \mathrm{BFGGI}\end{array}$ $\begin{array}{lllllll}642 & 1368 F 4 & 16 & 150 & 320 & 2 & \text { BFGGI }\end{array}$ $643 \quad 14 \mathrm{AC7} 716 \quad 150 \quad 320 \quad 2 \quad$ BFGGI $644 \quad 143 F 68 \quad 16 \quad 150 \quad 320 \quad 2 \quad$ BFGGJ 645 14BA97 $16 \quad 150320 \quad 2 \quad$ BFGG J $646 \quad 1437 \mathrm{~EB} \quad 16 \quad 1503202$ BFGHI 647 143ECB $16 \quad 150320 \quad 2 \quad$ BFGHI $648 \quad 143 F 6 \mathrm{~A} \quad 16 \quad 150320 \quad 2 \quad \mathrm{BFGHI}$ 649 143FDA $16 \quad 150320 \quad 2 \quad$ BFGHI 650 14BCE9 161503202 BFGHI

$$
\begin{array}{lllllll}
N & S P & S & H_{1} & H_{2} & H_{3} & L T
\end{array}
$$

$\begin{array}{lllllll}651 & 1569 F A & 16 & 150 & 320 & 2 & \text { BFGHI }\end{array}$ $\begin{array}{lllllll}652 & 143 \mathrm{~A} 6 \mathrm{~F} & 16 & 150 & 320 & 2 & \mathrm{BFGHJ}\end{array}$ 653 143CAF $16 \quad 150320 \quad 2 \quad$ BFGHJ 654 143DBE $16 \quad 1503202$ BFGHJ $\begin{array}{lllllll}655 & 14 B 79 C & 16 & 150 & 320 & 2 & \text { BFGHJ }\end{array}$ 656 14B9EC $16 \quad 150320 \quad 2 \quad$ BFGHJ $\begin{array}{lllllll}657 & 1357 \mathrm{~F} 9 & 16 & 150 & 320 & 2 & \mathrm{BFGII}\end{array}$ $\begin{array}{lllllll}658 & 136 \mathrm{AD} 4 & 16 & 150 & 320 & 2 & \mathrm{BFGII}\end{array}$ $\begin{array}{lllllll}659 & 1367 D 8 & 16 & 150 & 320 & 2 & \text { BFGIJ }\end{array}$ $\begin{array}{lllllll}660 & 1367 \mathrm{FA} & 16 & 150 & 320 & 2 & \mathrm{BFGIJ}\end{array}$ 661 13D7B8 $16 \quad 150320 \quad 2 \quad$ BFGIJ 662 143C6B $16 \quad 150320 \quad 2$ BFGIJ $\begin{array}{lllllll}663 & 153 \mathrm{CAF} & 16 & 150 & 320 & 2 & \mathrm{BFGIJ}\end{array}$ $\begin{array}{lllllll}664 & 14396 \mathrm{E} & 16 & 150 & 320 & 2 & \mathrm{BFGJJ}\end{array}$ 665 14B7CE $16 \quad 1503202$ BFGJJ 666 15BF9C $16 \quad 150320 \quad 2 \quad$ BFGJJ $\begin{array}{lllllll}667 & 156 \mathrm{ADE} & 16 & 150 & 320 & 2 & \mathrm{BFHHJ}\end{array}$ 668 157BDF $16 \quad 150320 \quad 2 \quad$ BFHII 669 13D7A9 $16 \quad 1503202$ BFIII $\begin{array}{lllllll}670 & 135 B 7 D & 16 & 150 & 320 & 2 & \text { FFGGH }\end{array}$ $\begin{array}{lllllll}671 & 13587 \mathrm{E} & 16 & 150 & 320 & 2 & \mathrm{FFGGJ}\end{array}$ $\begin{array}{lllllll}672 & 13597 \mathrm{~F} & 16 & 150 & 320 & 2 & \text { FFGHI }\end{array}$ $\begin{array}{lllllll}673 & 153 \mathrm{C} 7 \mathrm{~A} & 16 & 150 & 320 & 2 & \mathrm{FFGII}\end{array}$ $\begin{array}{lllllll}674 & 135 A 7 C & 16 & 150 & 320 & 2 & \text { FFGIJ }\end{array}$ $\begin{array}{lllllll}675 & 14 A B 97 & 16 & 150 & 230 & 1 & \text { AGJJJ }\end{array}$ $\begin{array}{lllllll}676 & 157 \mathrm{CBF} & 16 & 150 & 230 & 1 & \text { AHIIJ }\end{array}$ $\begin{array}{lllllll}677 & 13 E F 85 & 16 & 150 & 230 & 1 & \text { AHJJJ }\end{array}$ $\begin{array}{lllllll}678 & 13 D B 64 & 16 & 150 & 230 & 1 & \text { BGGHJ }\end{array}$ $\begin{array}{lllllll}679 & 13 E E 94 & 16 & 150 & 230 & 1 & \text { BGGHJ }\end{array}$ $\begin{array}{lllllll}680 & 143 C E B & 16 & 150 & 230 & 1 & \text { BGGIJ }\end{array}$ 681 13C9F4 $16 \quad 150230 \quad 1$ BGHHI 682 13EED6 $16 \quad 150230 \quad 1 \quad$ BGHHJ 683 13EF94 $16 \quad 150230 \quad 1 \quad$ BGHHJ $\begin{array}{lllllll}684 & 147 \mathrm{ADF} & 16 & 150 & 230 & 1 & \mathrm{BGHHJ}\end{array}$ $\begin{array}{lllllll}685 & 136 \mathrm{FC} 9 & 16 & 150 & 230 & 1 & \mathrm{BGHII}\end{array}$ $\begin{array}{lllllll}686 & 13 D B 65 & 16 & 150 & 230 & 1 & \text { BGHII }\end{array}$ $\begin{array}{lllllll}687 & 13 E A D & 16 & 150 & 230 & 1 & \text { BGHII }\end{array}$ 688 13EFC9 $16 \quad 150230 \quad 1$ BGHII $\begin{array}{lllllll}689 & 157 \mathrm{CFA} & 16 & 150 & 230 & 1 & \mathrm{BGHII}\end{array}$ $\begin{array}{lllllll}690 & 1357 F D & 16 & 150 & 230 & 1 & \text { BGHIJ }\end{array}$ $\begin{array}{lllllll}691 & 136 \mathrm{BDF} & 16 & 150 & 230 & 1 & \text { BGHIJ }\end{array}$ $\begin{array}{llllll}692 & 136 \mathrm{CEB} & 16 & 150 & 230 & 1 \\ 693 & \text { BGHIJ }\end{array}$ $\begin{array}{lllllll}693 & 136 \mathrm{~F} 94 & 16 & 150 & 230 & 1 & \text { BGHIJ }\end{array}$ $\begin{array}{lllllll}694 & 13 C A 74 & 16 & 150 & 230 & 1 & \text { BGHIJ }\end{array}$ 695 13D7A8 $16 \quad 150230 \quad 1$ BGHIJ 696 13ECA9 $16 \quad 150230 \quad 1$ BGHIJ $\begin{array}{lllllll}697 & 13 E E 6 B & 16 & 150 & 230 & 1 & \text { BGHIJ }\end{array}$ 698 13EED9 $16 \quad 150230 \quad 1 \quad$ BGHIJ $\begin{array}{lllllll}699 & 13 E F C 7 & 16 & 150 & 230 & 1 & \text { BGHIJ }\end{array}$ 700 1579EA $16 \quad 150230 \quad 1$ BGHIJ $\begin{array}{lllllll}701 & 15 A D E 6 & 16 & 150 & 230 & 1 & \text { BGHIJ }\end{array}$ $\begin{array}{lllllll}702 & 13 C C A 5 & 16 & 150 & 230 & 1 & \text { BGHJJ }\end{array}$ $\begin{array}{lllllll}703 & 13 C C E 7 & 16 & 150 & 230 & 1 & \text { BGHJJ }\end{array}$ 704 13DCB4 $16 \quad 150230 \quad 1 \quad$ BGHJJ 705 13DDE6 $16 \quad 1502301$ BGHJJ
$\begin{array}{lllllll}N & S P & S & H_{1} & H_{2} & H_{3} & L T\end{array}$

706 143F6C $16 \quad 150230 \quad 1$ BGHJJ $\begin{array}{lllllll}707 & 14 F 96 C & 16 & 150 & 230 & 1 & \text { BGHJJ }\end{array}$ 708 157F39 $16 \quad 150230 \quad 1 \quad$ BGHJJ 709 35AFE6 $16 \quad 150230 \quad 1$ BGHJJ 710 153CEA $16 \quad 150230 \quad 1$ BGIII 711 136DEB $16 \quad 150230 \quad 1$ BGIIJ $\begin{array}{lllllll}712 & 13 C A 75 & 16 & 150 & 230 & 1 & \text { BGIIJ }\end{array}$ 713 13D9E4 $16 \quad 150230 \quad 1 \quad$ BGIIJ 714 13EA9C $16 \quad 150230 \quad 1$ BGIIJ 715 15A9FD $16 \quad 150230 \quad 1 \quad$ BGIIJ $\begin{array}{lllllll}716 & 15 A C 36 & 16 & 150 & 230 & 1 & \text { BGIIJ }\end{array}$ $\begin{array}{lllllll}717 & 1357 \mathrm{EC} & 16 & 150 & 230 & 1 & \text { BGIJJ }\end{array}$ 718 136DB4 $16 \quad 150230 \quad 1 \quad$ BGIJJ 719 136EC9 $16 \quad 150230 \quad 1 \quad$ BGIJJ $720 \quad$ 13CDE7 $16 \quad 150230 \quad 1 \quad$ BGIJJ 721 13EBD5 $16 \quad 150230 \quad 1$ BGIJJ 722 13EEC9 $16 \quad 150230 \quad 1$ BGIJJ 723 156F9B $16 \quad 150230 \quad 1$ BGIJJ 724 15AE9D $16 \quad 150230 \quad 1$ BGIJJ 725 13EEC7 $16 \quad 150230 \quad 1 \quad$ BGJJJ $\begin{array}{lllllll}726 & 143 \mathrm{C} 6 \mathrm{~F} & 16 & 150 & 230 & 1 & \mathrm{BGJJJ}\end{array}$ 727 15B9ED $16 \quad 150230 \quad 1 \quad$ BGJJJ 728 136CFB $16 \quad 150230 \quad 1$ BHHHI 729 13DA75 $16 \quad 150230 \quad 1 \quad$ BHHII 730 13EFD6 $16 \quad 150230 \quad 1$ BHHII 731 136ED9 $16 \quad 150230 \quad 1$ BHHIJ 732 13D9E5 $16 \quad 150230 \quad 1 \quad$ BHHIJ 733 13DCE7 $16 \quad 150230 \quad 1 \quad$ BHHIJ 734 13DCF6 $16 \quad 150230 \quad 1$ BHHIJ $\begin{array}{lllllll}735 & 367 F 9 A & 16 & 150 & 230 & 1 & \text { BHHIJ }\end{array}$ $\begin{array}{lllllll}736 & 13 D C A 5 & 16 & 150 & 230 & 1 & \text { BHHJJ }\end{array}$ 737 156E3A $16 \quad 150230 \quad 1$ BHHJJ $\begin{array}{lllllll}738 & 357 \mathrm{ECF} & 16 & 150 & 230 & 1 & \text { BHIII }\end{array}$ $\begin{array}{lllllll}739 & 35 A D 69 & 16 & 150 & 230 & 1 & \text { BHIII }\end{array}$ 740 1369FD $16 \quad 150230 \quad 1$ BHIIJ 741 13DA65 $16 \quad 150230 \quad 1$ BHIIJ 742 13DCE6 $16 \quad 150230 \quad 1$ BHIIJ $\begin{array}{lllllll}743 & 13 E 975 & 16 & 150 & 230 & 1 & \text { BHIIJ }\end{array}$ $\begin{array}{lllllll}744 & 13 E D B 9 & 16 & 150 & 230 & 1 & \text { BHIIJ }\end{array}$ $\begin{array}{lllllll}745 & 156 \mathrm{C} 3 \mathrm{~A} & 16 & 150 & 230 & 1 & \text { BHIIJ }\end{array}$ $\begin{array}{lllllll}746 & 15 A D 36 & 16 & 150 & 230 & 1 & \text { BHIIJ }\end{array}$ 747 357ACE $16 \quad 150230 \quad 1$ BHIIJ $\begin{array}{lllllll}748 & 357 \mathrm{BDF} & 16 & 150 & 230 & 1 & \text { BHIIJ }\end{array}$ $\begin{array}{lllllll}749 & 367 \mathrm{BDE} & 16 & 150 & 230 & 1 & \text { BHIIJ }\end{array}$ 750 136ADE $16 \quad 150230 \quad 1$ BHIJJ 751 13EAD5 $16 \quad 150230 \quad 1$ BHIJJ $\begin{array}{lllllll}752 & 13 E D 75 & 16 & 150 & 230 & 1 & \text { BHIJJ }\end{array}$ 753 13EF7B $16 \quad 150230 \quad 1$ BHIJJ 754 13EFD7 $16 \quad 150230 \quad 1 \quad$ BHIJJ $\begin{array}{lllllll}755 & 153 \mathrm{~A} 6 \mathrm{E} & 16 & 150 & 230 & 1 & \mathrm{BHIJJ}\end{array}$ 756 153B6F $16 \quad 150230 \quad 1$ BHIJJ 757 153DAE $16 \quad 150230 \quad 1$ BHIJJ $\begin{array}{lllllll}758 & 156 F 39 & 16 & 150 & 230 & 1 & \text { BHIJJ }\end{array}$ $\begin{array}{lllllll}759 & 357 \mathrm{FDE} & 16 & 150 & 230 & 1 & \text { BHIJJ }\end{array}$ 760 157ADE $16 \quad 150230 \quad 1$ BHJJJ

TABLE 3 (continued). 


\section{$\begin{array}{lllllll}N & S P & S & H_{1} & H_{2} & H_{3} & L T\end{array}$}

$\begin{array}{lllllll}761 & 1367 \mathrm{FB} & 16 & 150 & 230 & 1 & \mathrm{BIIJJ}\end{array}$ $\begin{array}{lllllll}762 & 136 \mathrm{DFB} & 16 & 150 & 230 & 1 & \text { BIIJJ }\end{array}$ 763 153C6F $16 \quad 150230 \quad 1 \quad$ BIIJJ 764 153D6E $16 \quad 150230 \quad 1$ BIIJJ 765 15AF36 $16 \quad 150230 \quad 1$ BIIJJ $\begin{array}{lllllll}766 & 13 E 9 B 7 & 16 & 150 & 230 & 1 & \text { BIJJJ }\end{array}$ $\begin{array}{lllllll}767 & 15 \mathrm{AE} 36 & 16 & 150 & 230 & 1 & \text { BIJJJ }\end{array}$ $\begin{array}{lllllll}768 & 136 \mathrm{CB} 4 & 16 & 150 & 230 & 1 & \text { FGGHI }\end{array}$ $\begin{array}{lllllll}769 & 14 B C 69 & 16 & 150 & 230 & 1 & \text { FGGHI }\end{array}$ 770 14BF9C $16 \quad 150230 \quad 1$ FGGHJ 771 13E79A $16 \quad 150230 \quad 1$ FGGII $\begin{array}{lllllll}772 & 136 \mathrm{E} 94 & 16 & 150 & 230 & 1 & \text { FGGIJ }\end{array}$ 773 13EB9C $16 \quad 150230 \quad 1$ FGGIJ 774 35FAB6 $16 \quad 150230 \quad 1 \quad$ FGHHI 775 15BA96 $16 \quad 150230 \quad 1$ FGHHJ $\begin{array}{lllllll}776 & 156 \mathrm{DBE} & 16 & 150 & 230 & 1 & \text { FGHII }\end{array}$ $\begin{array}{lllllll}777 & 15 A D E 9 & 16 & 150 & 230 & 1 & \text { FGHII }\end{array}$ $\begin{array}{lllllll}778 & 1357 \mathrm{ED} & 16 & 150 & 230 & 1 & \text { FGHIJ }\end{array}$ $\begin{array}{lllllll}779 & 13679 \mathrm{C} & 16 & 150 & 230 & 1 & \text { FGHIJ }\end{array}$ $\begin{array}{lllllll}780 & 1367 \mathrm{BE} & 16 & 150 & 230 & 1 & \text { FGHIJ }\end{array}$ $\begin{array}{lllllll}781 & 157 \mathrm{ACE} & 16 & 150 & 230 & 1 & \text { FGHIJ }\end{array}$ 782 157E3A $16 \quad 150230 \quad 1$ FGHIJ 783 15AF9C $16150230 \quad 1$ FGHIJ $\begin{array}{lllllll}784 & 153 \mathrm{~A} 7 \mathrm{E} & 16 & 150 & 230 & 1 & \text { FGHJJ }\end{array}$ $\begin{array}{lllllll}785 & 153 B 7 F & 16 & 150 & 230 & 1 & \text { FGHJJ }\end{array}$ $\begin{array}{lllllll}786 & 156 \mathrm{E} 9 \mathrm{~A} & 16 & 150 & 230 & 1 & \mathrm{FGHJ} J\end{array}$ $\begin{array}{lllllll}787 & 1367 C 9 & 16 & 150 & 230 & 1 & \text { FGIIJ }\end{array}$ $\begin{array}{lllllll}788 & 1367 \mathrm{~EB} & 16 & 150 & 230 & 1 & \text { FGIIJ }\end{array}$ $\begin{array}{lllllll}789 & 13 C 7 A 9 & 16 & 150 & 230 & 1 & \text { FGIIJ }\end{array}$ $790 \quad 157 \mathrm{CAF} \quad 16 \quad 150230 \quad 1 \quad$ FGIIJ 791 15BE36 $16 \quad 150230 \quad 1$ FGIIJ $\begin{array}{lllllll}792 & 367 \mathrm{AFB} & 16 & 150 & 230 & 1 & \text { FGIIJ }\end{array}$ 793 1357FC $16 \quad 150230 \quad 1 \quad$ FGIJJ 794 153C7F $16 \quad 150230 \quad 1 \quad$ FGIJJ 795 153D7E $16 \quad 150230 \quad 1$ FGIJJ 796 157F9B $16 \quad 150230 \quad 1$ FGIJJ 797 15EDA6 $16 \quad 150230 \quad 1 \quad$ FGIJJ 798 367C9D $16 \quad 150230 \quad 1$ FHHII $\begin{array}{lllllll}799 & 367 \mathrm{BEA} & 16 & 150 & 230 & 1 & \text { FHHIJ }\end{array}$ $800 \quad 157 F 8 B \quad 16 \quad 150221 \quad 1 \quad$ AJJJJ 801 13EFD8 $16 \quad 150221 \quad 1 \quad$ BGHIJ 802 1368FC $16 \quad 150221 \quad 1 \quad$ BGHJJ 803 13EFD9 $16 \quad 150221 \quad 1$ BHIII 804 136FD9 $16 \quad 150221 \quad 1 \quad$ BHIIJ 805 35AFEC $16 \quad 150221 \quad 1$ BHIIJ 806 357FAE $16 \quad 150221 \quad 1$ BHIJ J 807 1367D9 $16150221 \quad 1$ BIIJ J $808 \quad 357$ AC9 $16 \quad 150221 \quad 1$ FHHII 809 357AFC $16 \quad 150221 \quad 1 \quad$ FHHII 810 35FB9D $16 \quad 150221 \quad 1$ FHIIJ 811 14BA69 $16150140 \quad 0$ GGIJJ 812 13EE95 $16150140 \quad 0 \quad$ GGJJJ 813 13CDF6 $16150140 \quad 0$ GHIIJ 814 13EF6B $16 \quad 150140 \quad 0$ GHIIJ 815 13EC65 $16 \quad 150140 \quad 0$ GHIJ J
$\begin{array}{lllllll}N & S P & S & H_{1} & H_{2} & H_{3} & L T\end{array}$

816 157DAE $16 \quad 150140 \quad 0$ GHIJJ $817 \quad 15 A B 96 \quad 16 \quad 150140 \quad 0$ GHIJJ 818 15BA69 $16 \quad 150140 \quad 0$ GHIJJ 819 13EF95 $16150140 \quad 0$ GHJJJ 820 15BE9D $16150140 \quad 0$ GHJJJ 821 15BD69 $16150140 \quad 0$ GIIIJ 822 367AC5 $16 \quad 150140 \quad 0$ GIIIJ 823 367ECF $16150140 \quad 0$ GIIIJ 824 13C9F5 $16150140 \quad 0$ GIIJJ 825 13ED65 $16150140 \quad 0$ GIIJJ 826 13EDA9 $16 \quad 150140 \quad 0$ GIIJJ 827 15ACE6 $16 \quad 150140 \quad 0$ GIIJJ 82815 AD79 $16 \quad 150140 \quad 0$ GIIJJ 829 367DB5 $16150140 \quad 0$ GIIJJ $830 \quad 367$ EB9 $16 \quad 150140 \quad 0$ GIIJJ $831 \quad 13 C D F 7 \quad 16 \quad 150140 \quad 0$ GIJJJ $832 \quad 157 \mathrm{E} 9 \mathrm{~A} \quad 16 \quad 150140 \quad 0$ GIJJJ 833 13CDA5 $16150140 \quad 0$ GJJJJ 834 367EFD $16150140 \quad 0$ HHIII 835 367DFA $16 \quad 150140 \quad 0$ HHIJJ $836 \quad 367 \mathrm{EF} 516 \quad 150140 \quad 0$ HIIII 837 367EC5 $16 \quad 150140 \quad 0$ HIIIJ 838 357AFB $16150140 \quad 0$ HIIJJ 839 357BEA $16 \quad 150140 \quad 0$ HIIJJ 840 35ACE6 $16 \quad 150140 \quad 0$ HIIJJ 841 35ACED $16150140 \quad 0$ HIIJJ 842 367F95 $16150140 \quad 0$ HIIJJ 843 35ADCE $16150140 \quad 0$ HIJJJ 844 367C9B $16 \quad 150140 \quad 0$ HIJJJ 845 367FD5 $16 \quad 150140 \quad 0$ HIJJJ 846 367BEC $16 \quad 150140 \quad 0$ HJJJJ 847 367DFC $16150140 \quad 0$ IIJJJ 848 367FDE $16150140 \quad 0$ IIJJJ $849 \quad 13586 \mathrm{~F} \quad 16 \quad 150131 \quad 0 \quad$ GGHJJ 850 135B6C $16 \quad 150131 \quad 0 \quad$ GGJJJ 851 13E79B $16 \quad 150131 \quad 0$ GHIIJ 852 135A6D $16 \quad 150131 \quad 0$ GHIJJ 853 157BCF $16 \quad 150131 \quad 0$ GHIJJ 854 15EF6C $16 \quad 150131 \quad 0$ GHJJJ $\begin{array}{lllllll}855 & 13596 \mathrm{E} & 16 & 150 & 131 & 0 & \text { GIJJJ }\end{array}$ 856 13679D $16 \quad 150131 \quad 0 \quad$ GIJJJ $857 \quad 1367 \mathrm{BF} \quad 16 \quad 150131 \quad 0$ GIJJJ 858 35ADC9 $16 \quad 150131 \quad 0$ HIIIJ 859 35FAB9 $16 \quad 150131 \quad 0$ HIIIJ $860367 D C 5 \quad 16 \quad 150131 \quad 0$ HIIIJ 861 367DCE 16150131 0 HIIIJ $862 \quad 367 E C 916150131 \quad 0$ HIIIJ 863 367BD5 $16 \quad 150131 \quad 0$ HIIJJ 864 367DF5 $16 \quad 150131 \quad 0 \quad$ HIIJJ 865 35ABE6 $16 \quad 150131 \quad 0$ HIJJJ 866 14A86F 321414103 AABJJ 867 15BF8C $32141410 \quad 3 \quad$ ABBJJ 868 143B6C $16 \quad 141 \quad 410 \quad 3 \quad$ ABFHJ 869 156CAF $16 \quad 141410 \quad 3 \quad$ ABFII 870 156F8B $16 \quad 1414103$ ABFJJ
$\begin{array}{lllllll}N & S P & S & H_{1} & H_{2} & H_{3} & L T\end{array}$ 871 136CF4 $16 \quad 141320 \quad 2$ ABHIJ $872 \quad$ 136ED4 $16 \quad 141320 \quad 2$ ABIJJ 873 14BA79 $16 \quad 141320 \quad 2$ AFGIJ $\begin{array}{lllllll}874 & 13486 \mathrm{~F} & 16 & 141 & 320 & 2 & \mathrm{AFGJJ}\end{array}$ 875 13E78C $16 \quad 141320 \quad 2$ AFIIJ $\begin{array}{lllllll}876 & 15 B D 79 & 16 & 141 & 320 & 2 & \text { AFIIJ }\end{array}$ $\begin{array}{lllllll}877 & 134 \mathrm{~A} 6 \mathrm{D} & 16 & 141 & 320 & 2 & \mathrm{AFIJJ}\end{array}$ 878 157DBE $16 \quad 141320 \quad 2$ AFIJJ 879 156DEB $16 \quad 141320 \quad 2 \quad$ BBGGI 880 1479FA $16 \quad 141 \quad 320 \quad 2 \quad$ BFGGH 881 13C7E8 $16 \quad 141320 \quad 2 \quad$ BFGGI 882 13E7CA $16 \quad 141320 \quad 2 \quad$ BFGGI $\begin{array}{lllllll}883 & 14 F 97 C & 16 & 141 & 320 & 2 & \text { BFGGJ }\end{array}$ $\begin{array}{lllllll}884 & 1357 \mathrm{BD} & 16 & 141 & 320 & 2 & \mathrm{BFGHI}\end{array}$ 885 136FD4 $16 \quad 141320 \quad 2 \quad$ BFGHI $\begin{array}{lllllll}886 & 13 E 7 B 8 & 16 & 141 & 320 & 2 & \text { BFGHI }\end{array}$ $\begin{array}{lllllll}887 & 143 A 6 D & 16 & 141 & 320 & 2 & B F G H J\end{array}$ $\begin{array}{lllllll}888 & 156 B C F & 16 & 141 & 320 & 2 & \text { BFGHJ }\end{array}$ $\begin{array}{llllll}889 & 1357 \mathrm{AC} & 16 & 141 & 320 & 2 \\ 8 F G I J\end{array}$ 890 136DF4 $16 \quad 141320 \quad 2 \quad$ BFGIJ

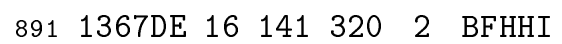
$892 \quad 1367 \mathrm{FC} \quad 16 \quad 141320 \quad 2 \quad$ BFHHI 893 357F9C $16 \quad 141320 \quad 2 \quad$ BFHHI 894 13E9BC $16 \quad 141320 \quad 2$ BFHII 895 14B93E $16 \quad 141 \quad 320 \quad 2 \quad$ FFGGJ $\begin{array}{lllllll}896 & 15397 \mathrm{~F} & 16 & 141 & 320 & 2 & \text { FFGGJ }\end{array}$ $\begin{array}{lllllll}897 & 153 \mathrm{~A} 7 \mathrm{C} & 16 & 141 & 320 & 2 & \mathrm{FFGHH}\end{array}$ 898 157DFB $16 \quad 141230 \quad 1$ AGIIJ $\begin{array}{lllllll}899 & 367 \mathrm{ACF} & 16 & 141 & 230 & 1 & \text { BGGII }\end{array}$ 900 1368ED $16 \quad 141230 \quad 1 \quad$ BGGIJ 901 13C7EA $16 \quad 141230 \quad 1$ BGGIJ 902 13C8E5 $16 \quad 141230 \quad 1 \quad$ BGGJJ 903 13E8A7 $16 \quad 141230 \quad 1 \quad$ BGGJJ 904 14BA3D $16 \quad 141230 \quad 1 \quad$ BGGJJ 905 13E8BC $16 \quad 141230 \quad 1$ BGHIJ $\begin{array}{lllllll}906 & 136 \mathrm{ACF} & 16 & 141 & 230 & 1 & \text { BGIIJ }\end{array}$ 907 13E9A7 $16 \quad 141230 \quad 1$ BGIIJ $\begin{array}{lllllll}908 & 157 \mathrm{C} 3 \mathrm{~A} & 16 & 141 & 230 & 1 & \text { BGIIJ }\end{array}$ 909 357EBF $16 \quad 141230 \quad 1 \quad$ BGIIJ $\begin{array}{lllllll}910 & 367 C D 9 & 16 & 141 & 230 & 1 & \text { BGIIJ }\end{array}$

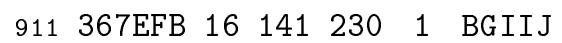
912 13EAC5 $16 \quad 141230 \quad 1 \quad$ BGIJJ 913 157D3B $16 \quad 141230 \quad 1 \quad$ BGIJJ 914 13E7B9 $16 \quad 141230 \quad 1$ BHIIJ 915 1367DF $16 \quad 141230 \quad 1$ BHIJJ $\begin{array}{lllllll}916 & 13 C 7 F A & 16 & 141 & 230 & 1 & \text { FGGHI }\end{array}$ 917 13E7A9 $16 \quad 141230 \quad 1 \quad$ FGGII 918 15BD36 $16 \quad 141230 \quad 1$ FGGII 919367 AC9 $16 \quad 141230 \quad 1$ FGGII 920 367EBF $16 \quad 141230 \quad 1 \quad$ FGGII 921 153DBE $16 \quad 141230 \quad 1 \quad$ FGGIJ 922 1579FA $16 \quad 141230 \quad 1$ FGGIJ 923 15A9ED $16 \quad 141230 \quad 1 \quad$ FGGIJ 924 15B9FD $16 \quad 141230 \quad 1 \quad$ FGGIJ 925 367DBE $16 \quad 141230 \quad 1$ FGGIJ

TABLE 3 (continued). 


\section{$\begin{array}{lllllll}N & S P & S & H_{1} & H_{2} & H_{3} & L T\end{array}$}

926 15A9E6 $16 \quad 141230 \quad 1 \quad$ FGGJ J $927 \quad 13 E 96 C \quad 16 \quad 141230 \quad 1$ FGHII $928 \quad 1367 \mathrm{CF} \quad 16 \quad 141230 \quad 1 \quad$ FGHIJ 929 1367ED $16 \quad 141230 \quad 1$ FGHIJ 930 157F3B $16 \quad 141230 \quad 1$ FGHJJ 931 367F9C $16 \quad 141230 \quad 1$ FGHJJ 932 15BF36 $16 \quad 141230 \quad 1$ FGIJJ 933 13EB6C $16 \quad 141221 \quad 1 \quad$ BGGIJ 934 13C7F9 $16141221 \quad 1 \quad$ BGIIJ 935 1367FD $16 \quad 141221 \quad 1 \quad$ BHIJJ 936 35ADF9 $16 \quad 141221 \quad 1 \quad$ FGGHI 937 13EBC6 $16 \quad 141221 \quad 1 \quad$ FGGHJ 938 13C9E6 $16 \quad 141221 \quad 1 \quad$ FGHIJ

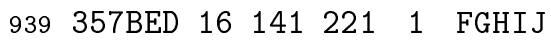
$940 \quad 13 C 7 A E \quad 16 \quad 141221 \quad 1 \quad$ FGIIJ

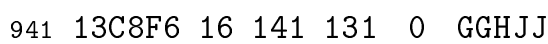
$942 \quad 13 E 86516 \quad 141 \quad 131 \quad 0 \quad$ GGIIJ 943 13CB65 $16141131 \quad 0$ GGIJJ 944 136BCF $16 \quad 141 \quad 131 \quad 0$ GGJJJ 945 13EBC5 $16 \quad 141 \quad 131 \quad 0$ GGJJJ 946 13ED6A $16 \quad 141 \quad 131 \quad 0 \quad$ GGJJJ 947 367CDF $16 \quad 141 \quad 131 \quad 0$ GHIIJ 948 153CBF $16 \quad 141 \quad 131 \quad 0$ GHIJJ 949 367AFD $16 \quad 141 \quad 131 \quad 0$ GHIJJ 950 13EDA6 $16 \quad 141 \quad 131 \quad 0$ GHJJJ 951 15EBC6 $16 \quad 141 \quad 131 \quad 0$ GHJJJ 952 13C7FB $16 \quad 141 \quad 131 \quad 0$ GIIIJ 953 13CA65 $16141131 \quad 0$ GIIIJ 954 13E965 $16 \quad 141 \quad 131 \quad 0$ GIIJJ $\begin{array}{lllllll}955 & 1369 E D & 16 & 141 & 131 & 0 & \text { GIJJJ }\end{array}$ 956 13C9E5 $16 \quad 141 \quad 131 \quad 0$ GIJJJ 957 367CD5 $16141131 \quad 0$ HIIIJ 958 367CE5 $16 \quad 141 \quad 131 \quad 0$ HIIJJ 959 367CED $16 \quad 141 \quad 122 \quad 0$ HIIJJ 960 14A7F9 $16 \quad 060400 \quad 4 \quad$ BBBFI 961 14F7A9 $16 \quad 060400 \quad 4 \quad$ BBBF J 962 14A79F $16 \quad 060310 \quad 3 \quad$ BBBG J 963 14FD6A $16 \quad 060310 \quad 3 \quad$ BBBJ J 964 15EC7A $16 \quad 060310 \quad 3 \quad$ BBFIJ 965 14F79A $16 \quad 060310 \quad 3 \quad$ BFFGJ 966 1BFFD8 $32060220 \quad 2 \quad$ BBGJJ 967 1B6FD8 $32060220 \quad 2 \quad$ BBHJJ 968 1C6F38 $32060220 \quad 2 \quad$ BBHJJ 969 14A7DF $16060220 \quad 2 \quad$ BBJJJ 970 14B7EC $16060220 \quad 2$ BBJJJ 971 14BE69 $16060220 \quad 2 \quad$ BFGIJ 972 14BCE7 $16 \quad 060220 \quad 2 \quad$ BFGJ J 973 14BD6E $16 \quad 060220 \quad 2 \quad$ BFGJJ 974 17BE9D $16060220 \quad 2 \quad$ BFGJJ 975 15BCE6 $16060220 \quad 2$ BFHII 976 15BDE6 $16060220 \quad 2$ BFHII 977 35AEFD $16060220 \quad 2$ BFHII 978 15EF7C $16060220 \quad 2 \quad$ BFHJJ 979 15FDA6 $16060220 \quad 2 \quad$ BFIJJ 980 14AC7F $16 \quad 060220 \quad 2 \quad$ FFGGJ
$\begin{array}{lllllll}N & S P & S & H_{1} & H_{2} & H_{3} & L T\end{array}$

981 14BF97 $16 \quad 060 \quad 220 \quad 2 \quad$ FFGGJ 982 17AF9C $16 \quad 060220 \quad 2 \quad$ FFGHJ 983 15BC6F $16 \quad 060220 \quad 2 \quad$ FFGIJ 984 16BF9C $16 \quad 060220 \quad 2 \quad$ FFGJJ 985 57EC6D $96 \quad 060220 \quad 2 \quad$ FFHHH $\begin{array}{lllllll}986 & 35 F A 6 B & 16 & 060 & 220 & 2 & \text { FFHHI }\end{array}$ 987 36ABE7 $16 \quad 060 \quad 220 \quad 2 \quad$ FFHHJ 988 14BF68 $16 \quad 060 \quad 130 \quad 1 \quad$ BGGJJ 989 15ACF6 $16 \quad 060 \quad 130 \quad 1 \quad$ BHIIJ 990 15ADF6 $16 \quad 060 \quad 130 \quad 1 \quad$ BHIIJ $\begin{array}{lllllll}991 & 36 \mathrm{CEBF} & 16 & 060 & 130 & 1 & \text { BIIIJ }\end{array}$ 992 36FEBD $16 \quad 060130 \quad 1$ BIIIJ 993 15ECB6 $16 \quad 060 \quad 130 \quad 1$ BIJJJ 994 15EFD6 $16 \quad 060130 \quad 1 \quad$ BIJJJ 995 15AD7E $16 \quad 060 \quad 130 \quad 1$ FGIJJ $996 \quad 15$ AF79 $16 \quad 060 \quad 130 \quad 1 \quad$ FGIJJ 997 16AE9D $16 \quad 060 \begin{array}{lllll}130 & 1 & \text { FGIJJ }\end{array}$ 998 15AE96 $16 \quad 060 \quad 130 \quad 1 \quad$ FGJJJ 999 15BE96 $16 \quad 060 \quad 130 \quad 1 \quad$ FGJJJ $1000 \quad 36$ AEF9 $16 \quad 060 \quad 130 \quad 1 \quad$ FHIII 1001 35ABED $16 \quad 060130 \quad 1$ FHIIJ 1002 35ADF6 $16 \quad 060130 \quad 1$ FHIIJ 1003 36ABD5 $16 \quad 060 \quad 130 \quad 1 \quad$ FHIJJ 1004 36AFD5 $16 \quad 060130 \quad 1 \quad$ FHIJJ 1005 37DE6F $16 \quad 060 \quad 130 \quad 1$ FHIJJ 1006 36ADF7 $16 \quad 060 \quad 130 \quad 1$ FHJJJ 1007 36AFE7 $16 \quad 060 \quad 130 \quad 1$ FHJJJ 1008 36CDB5 $16 \quad 060130 \quad 1$ FIIJJ 1009 36CEB9 $16 \quad 060130 \quad 1$ FIIJJ $1010 \quad 36$ CF97 $16 \quad 060 \quad 130 \quad 1 \quad$ FIIJJ 1011 14FF28 $320 \quad 060 \quad 040 \quad 0 \quad$ GGGGG 1012 DEFF7B $64 \quad 060040 \quad 0 \quad$ GJJJJ 1013 37DEBF $32060040 \quad 0 \quad$ HIIJJ 1014 36ADC5 $16 \quad 060040 \quad 0 \quad$ IIJJJ 1015 14FBD7 $16 \quad 051 \quad 310 \quad 3 \quad$ ABFJJ $1016 \quad 15 B C 7 F \quad 16 \quad 051310 \quad 3 \quad$ AFFIJ 1017 15BF79 $16 \quad 051 \quad 310 \quad 3 \quad$ AFFIJ 1018 15BF86 $16 \quad 051310 \quad 3 \quad$ AFFJJ 1019 13D7F9 $16 \quad 051310 \quad 3 \quad$ BBFII 1020 13EA7C $16 \quad 051310 \quad 3 \quad$ BBFII 1021 13EC7A $16 \quad 051310 \quad 3 \quad$ BFFGH 1022 15FD7B $16 \quad 051310 \quad 3 \quad$ BFFGI $1023 \quad 14 \mathrm{~A} 7 \mathrm{CF} \quad 16 \quad 051310 \quad 3 \quad \mathrm{BFFGJ}$ 1024 15EA7C $16 \quad 051310 \quad 3 \quad \mathrm{BFFHH}$ 1025 13D9F7 $16 \quad 051310 \quad 3 \quad$ BFFHI 1026 14FB6C $16 \quad 051220 \quad 2 \quad$ ABJJJ 1027 13D7E8 $16 \quad 051220 \quad 2 \quad$ BBGIJ 1028 13D7FB $16 \quad 051220 \quad 2 \quad$ BBIIJ 1029 13E97C $16 \quad 051220 \quad 2 \quad$ BBIIJ 1030 14B96E $16 \quad 051220 \quad 2 \quad$ BFGGJ 1031 13E7D8 $16 \quad 051220 \quad 2 \quad$ BFGHI 1032 15BCF6 $16 \quad 051220 \quad 2 \quad$ BFGHI 1033 13D8E7 $16 \quad 051220 \quad 2 \quad$ BFGHJ 1034 14FA6D $16 \quad 051220 \quad 2 \quad$ BFGHJ 1035 15FA7C 160512202 BFGHJ

\section{$\begin{array}{lllllll}N & S P & S & H_{1} & H_{2} & H_{3} & L T\end{array}$}

1036 13D7FA $16 \quad 051220 \quad 2 \quad$ BFGIJ 1037 13E87C $16 \quad 051220 \quad 2 \quad$ BFGIJ 1038 13D8F6 $16 \quad 051220 \quad 2 \quad$ BFGJJ 1039 13EBD7 $16 \quad 051220 \quad 2 \quad$ BFGJJ 1040 15B9E6 $16 \quad 051220 \quad 2 \quad$ BFGJJ 1041 1B57ED $16 \quad 051220 \quad 2 \quad$ BFGJJ 1042 1B57FD $16051220 \quad 2 \quad$ BFGJJ 1043 13ECB6 $16 \quad 051220 \quad 2 \quad$ BFHHJ 1044 13D7EB $16051220 \quad 2 \quad$ BFHII 1045 35AEF6 $16051220 \quad 2 \quad$ BFHIJ 1046 35AFDE 160512202 BFHIJ 1047 15EAD6 $16051220 \quad 2 \quad$ BFHJJ 1048 15EB7D $16 \quad 051220 \quad 2 \quad$ BFHJJ 1049 15FAD6 $16051220 \quad 2 \quad$ BFHJJ 1050 35AFD9 $16051220 \quad 2 \quad$ BFIII 1051 13D7AF $16 \quad 051220 \quad 2$ BFIIJ 1052 35FE6D $16051220 \quad 2$ BFIIJ 1053 13ED7B $16051220 \quad 2 \quad$ BFIJJ 1054 13EDB7 $16 \quad 051220 \quad 2 \quad$ BFJJJ 1055 15FB7D $16051220 \quad 2 \quad$ FFGHI 1056 13ECA7 $16 \quad 051220 \quad 2 \quad$ FFGHJ 1057 15FBC6 $16 \quad 051220 \quad 2 \quad$ FFGHJ 1058 15FCB6 $16 \quad 051220 \quad 2 \quad$ FFGIJ 1059 36FEB7 $16 \quad 051220 \quad 2$ FFIII 1060 13E7DB $16 \quad 051211 \quad 2 \quad$ BBIIJ 1061 13D7EA $16 \quad 051130 \quad 1 \quad$ BGIIJ 1062 14F96A $16 \quad 051 \quad 130 \quad 1 \quad$ BGJJJ 1063 13EAD6 $16051 \quad 130 \quad 1$ BHIJJ 1064 13D9E6 $16 \quad 051 \quad 130 \quad 1$ BIJJJ 1065 13EC6B $16 \quad 051 \quad 130 \quad 1 \quad$ FGHIJ $\begin{array}{lllllll}1066 & 36 \text { ACD9 } & 16 & 051 & 130 & 1 & \text { FGIII }\end{array}$ 1067 37AFD9 $16 \quad 051 \quad 130 \quad 1 \quad$ FGIII $\begin{array}{lllllll}1068 & 13 C 7 E B & 16 & 051 & 130 & 1 & \text { FGIIJ }\end{array}$ 1069 13E7C9 $16 \quad 051130 \quad 1$ FGIIJ 1070 15BDF6 $16 \quad 051130 \quad 1$ FGIIJ 1071 36CDBE $16 \quad 051 \quad 130 \quad 1$ FGIIJ $\begin{array}{lllllll}1072 & 13 C 9 F 7 & 16 & 051 & 130 & 1 & \text { FGIJJ }\end{array}$ 1073 15FE7D $16 \quad 051130 \quad 1$ FGIJJ 107437 ACDF $16 \quad 051 \quad 130 \quad 1$ FHIII 1075 36AEFD $16051130 \quad 1$ FIIJJ 1076 13E7D9 $16 \quad 051 \quad 121 \quad 1 \quad$ BIIIJ 1077 35AFE9 $16 \quad 051 \quad 121 \quad 1 \quad$ BIIJJ 1078 13E79C $16 \quad 051 \quad 121 \quad 1 \quad$ FGIIJ $\begin{array}{lllllll}1079 & 36 \mathrm{ACDF} & 16 & 051 & 121 & 1 & \text { FHIII }\end{array}$ 1080 36CFE7 $80051050 \quad 0 \quad$ JJJJJ 1081 37EDBC $64051031 \quad 0 \quad$ HJJJJ 1082 36CF9E $16 \quad 051031 \quad 0 \quad$ IIIJJ 1083 37ADC9 $16 \quad 051031 \quad 0 \quad$ IIIJJ 1084 37AFE9 $16 \quad 051 \quad 031 \quad 0 \quad$ IIIJJ 1085 15B97F $32 \quad 042 \quad 400 \quad 4 \quad$ ABFFG 1086 35FEBD 320422202 BBGII 1087 13C8E7 $16 \quad 042 \quad 220 \quad 2 \quad$ FFGGJ 1088 14F97A $16 \quad 042220 \quad 2 \quad$ FFGGJ 1089 13EAC7 $16 \quad 042 \quad 220 \quad 2 \quad$ FFGIJ 1090 36ACD5 $16 \quad 042121 \quad 1$ FGIIJ

TABLE 3 (conclusion). 


\begin{tabular}{|c|c|c|c|c|c|c|}
\hline$N$ & $S P$ & $S$ & $H_{1}$ & $H_{2}$ & & $L 1$ \\
\hline & $F_{3}$ & & 510 & 0 & 3 & GHH \\
\hline 92 & BFFCA & & 430 & 63 & & $a^{\prime}$ \\
\hline 93 & AA35 & 16 & 420 & 630 & & GHIJA \\
\hline 94 & BFF3A & & 420 & 540 & & AGHHGG \\
\hline 95 & FF3A & & 420 & & & HHHF \\
\hline 96 & C65 & 48 & 30 & 620 & & \\
\hline 97 & ЗАA9F & 16 & 330 & 440 & 1 & $\mathrm{AHIIG}$ \\
\hline 98 & 53FF36 & 16 & 30 & 440 & & AHIJGG \\
\hline 099 & 56FF35 & 16 & 30 & 440 & 1 & BGHHGG \\
\hline 00 & $56 \mathrm{FA} 65$ & 16 & 30 & 440 & & BGIJGG \\
\hline 01 & $56 \mathrm{AF} 35$ & 16 & 30 & 3 & & GHJJGG \\
\hline 02 & 3AA65 & 16 & 30 & 350 & 0 & HIIGG \\
\hline 03 & A & 16 & 30 & 350 & 0 & $\mathrm{HIJGG}$ \\
\hline 04 & 53FA65 & 16 & 30 & 350 & 0 & HHI JGG \\
\hline 05 & 53FA35 & 16 & 30 & 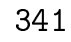 & 0 & GHHHGG \\
\hline 06 & $56 \mathrm{FF} 65$ & 16 & 21 & 6 & 3 & BHIJAA \\
\hline 107 & 53AF35 & 16 & 321 & 440 & & AGIJGG \\
\hline 108 & 5 & 16 & & 440 & & BGGHGG \\
\hline 09 & $56 \mathrm{AA}$ & 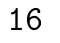 & & 350 & & GGJJGG \\
\hline 10 & 53FF9A & 16 & 25 & 430 & 2 & ABGIII \\
\hline 11 & 59FF9A & 32 & 50 & 30 & 2 & BBIIGG \\
\hline 112 & 53FF39 & 16 & 40 & 520 & 3 & AHI JBB \\
\hline 13 & $56 \mathrm{FFC5}$ & 16 & & 520 & & BGHHBB \\
\hline 14 & $53 \triangle \wedge$ & 1 & & 43 & & GH I JBB \\
\hline 15 & & & & 340 & & AIIJII \\
\hline 10 & & & & 340 & & BGHIII \\
\hline 17 & & & & & & {$[$ I } \\
\hline
\end{tabular}

\begin{tabular}{|c|c|c|c|c|c|c|}
\hline & $S P$ & $S$ & $H_{1}$ & $H_{2}$ & $H_{3}$ & $L 7$ \\
\hline 118 & $56 \mathrm{FFO}$ & $x$ & xu & 340 & 1 & BHIJ \\
\hline & & & 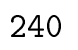 & & & \\
\hline & & 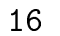 & 240 & 340 & & \\
\hline & CC65 & 6 & 240 & 340 & & \\
\hline & FAC5 & 10 & 240 & 250 & & HHJI \\
\hline & 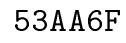 & & 40 & 250 & & 11 \\
\hline & $F F$ & & 40 & 250 & & 11 \\
\hline & AA36 & 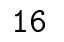 & 40 & 250 & & \\
\hline & & 16 & to & 50 & & JII \\
\hline & & 16 & 10 & 41 & & $\mathrm{GHHJI}$ \\
\hline & & & 31 & \pm 0 & & \\
\hline 29 & FF6A & & 31 & 40 & & AGH \\
\hline 30 & FAC5 & 6 & 31 & 40 & & 3GGJI \\
\hline & & & & 40 & & \\
\hline & & & & & & \\
\hline & & & & & & FGI \\
\hline & $n$ & & 2 & 250 & & HHJJGC \\
\hline 35 & $\mathrm{CC} 6 \mathrm{~F}$ & 6 & 150 & 20 & & IIJBB \\
\hline & FC65 & & 150 & 30 & & BFIII] \\
\hline 37 & & & 50 & 30 & & HI J \\
\hline & & & & & & \\
\hline & & 16 & 150 & 240 & & IT \\
\hline & $A C$ & & 150 & 240 & & BHIJII \\
\hline & & 10 & 150 & 240 & 1 & FHIJII \\
\hline & & & & 150 & & IHIIII \\
\hline & & & & & & \\
\hline & & & & & & \\
\hline
\end{tabular}

\begin{tabular}{|c|c|c|c|c|c|c|}
\hline$N$ & $S P$ & $S$ & $H_{1}$ & $H_{2}$ & $H_{3}$ & $L T$ \\
\hline 1145 & 56CCF5 & 16 & 141 & 420 & 3 & FHIIBB \\
\hline 1146 & $36 \mathrm{AAF} 9$ & 16 & 141 & 330 & 2 & BFGIII \\
\hline 147 & $35 \mathrm{AAF} 9$ & 16 & 141 & 330 & 2 & BFHIGG \\
\hline 1148 & $53 \mathrm{AC} 35$ & 16 & 141 & 330 & 2 & GHI JBB \\
\hline 1149 & $53 \mathrm{AF} 36$ & 16 & 141 & 240 & 1 & AIIJII \\
\hline 1150 & $56 \mathrm{CA} 6 \mathrm{~F}$ & 16 & 141 & 240 & 1 & BGHIII \\
\hline 1151 & $36 \mathrm{AACF}$ & 16 & 141 & 240 & 1 & BGHJII \\
\hline 1152 & $36 \mathrm{CC} 6 \mathrm{~F}$ & 16 & 141 & 240 & 1 & BIJJGG \\
\hline 1153 & $36 \mathrm{AF} 65$ & 16 & 141 & 240 & 1 & BIJJII \\
\hline 1154 & $56 \mathrm{CF} 35$ & 16 & 141 & 240 & 1 & FGHIII \\
\hline 1155 & $36 \mathrm{CF} 65$ & 16 & 141 & 240 & 1 & FIJJII \\
\hline 1156 & $56 \mathrm{AC} 35$ & 16 & 141 & 150 & 0 & GIJJII \\
\hline 1157 & $36 \mathrm{AAC5}$ & 16 & 141 & 150 & 0 & GJJJII \\
\hline 1158 & $53 \mathrm{AC} 36$ & 16 & 141 & 150 & 0 & HIIJII \\
\hline 1159 & $36 \mathrm{FF} 65$ & 16 & 132 & 330 & 2 & BFIJGG \\
\hline 1160 & $56 \mathrm{AAC} 9$ & 16 & 132 & 240 & 1 & FGGIII \\
\hline 1161 & $36 \mathrm{CC} 9 \mathrm{~F}$ & 32 & 070 & 230 & 2 & BBIIII \\
\hline 1162 & $35 \mathrm{FFAC}$ & 128 & 061 & 410 & 4 & BBBBGG \\
\hline 1163 & $35 \mathrm{AAFC}$ & 32 & 051 & 230 & 2 & FFHHII \\
\hline 1164 & $39 \mathrm{FF} 65$ & 16 & 051 & 230 & 2 & FFHIII \\
\hline 1165 & $36 \mathrm{FF} 95$ & 16 & 051 & 140 & 1 & BJJJII \\
\hline 1166 & $35 \mathrm{AAF} 6$ & 16 & 051 & 140 & 1 & FHJJII \\
\hline 1167 & $39 \mathrm{CC} 6 \mathrm{~F}$ & 32 & 051 & 050 & 0 & JJJJII \\
\hline 1168 & $35 \mathrm{FF} 6 \mathrm{C}$ & 16 & 042 & 320 & 3 & BBF JII \\
\hline 1169 & $36 \mathrm{FF} 6 \mathrm{~A}$ & 16 & 042 & 320 & 3 & FHJJBB \\
\hline 1170 & $36 \mathrm{FF} 6 \mathrm{C}$ & 32 & 042 & 230 & 2 & FFJ JGG \\
\hline & & & & & & \\
\hline
\end{tabular}

TABLE 4. Non-orientable, 6 cusped, minimal volume, integral, congruence 2 , hyperbolic 4-manifolds.

headed by $S P$ lists the side-pairing for the manifold in a coded form that is explained in Section 5. The column headed by $S$ lists the number of symmetries of the manifolds. All the manifolds have a subgroup of symmetries corresponding to $K^{4}$. Therefore, the number of symmetries is a multiple of 16 . The possible orders are $16,32,48,64,80,96,128$, and 320 . Only manifold number 1011 has a symmetry group of order 320 .

The column of Tables $2-4$ headed by $H_{i}$ lists the $i$-th homology groups of the manifolds with the 3 digit number $a b c$ representing $\mathbb{Z}^{a} \oplus \mathbb{Z}_{2}^{b} \oplus \mathbb{Z}_{4}^{c}$ and the single digit entry $a$ representing $\mathbb{Z}^{a}$.

The column headed by $L T$ lists the link types of the cusps of the manifolds. Here A, B, ..., J represent the 10 closed Euclidean 3-manifolds in the order given by Hantzsche and Wendt [1935]. The orientable manifolds are $\mathrm{A}, \ldots, \mathrm{F}$ with $\mathrm{A}$ the 3 -torus and $\mathrm{F}$ the Hantzsche-Wendt 3-manifold [Zimmermann 1990]. Only C, D, and E do not occur as links of cusps of our manifolds. The closed Euclidean 3-manifolds are identified by their homology
[Hantzsche and Wendt 1935]. Manifold 1162 is the hyperbolic 24-cell space in [Ratcliffe 1994, p. 510]. Tables 2-4 give some indication of the diversity of hyperbolic 4-manifolds of finite volume.

\section{ELECTRONIC AVAILABILITY}

Plain text files of Tables 1-4 are available at ftp:// math.vanderbilt.edu/users/tschantz/mantabs. The files are 3mantab.txt and 4mantab.txt.

\section{REFERENCES}

[Bianchi 1892] L. Bianchi, "Sui gruppi di sostituzioni lineari con coefficienti appartenenti a corpi quadratici immaginari", Math. Ann. 40 (1892), 332-412.

[Brunner et al. 1984] A. M. Brunner, M. L. Frame, Y. W. Lee, and N. J. Wielenberg, "Classifying torsion-free subgroups of the Picard group", Trans. Amer. Math. Soc. 282:1 (1984), 205-235.

[Coxeter and Whitrow 1950] H. S. M. Coxeter and G. J. Whitrow, "World-structure and non-Euclidean 
honeycombs", Proc. Roy. Soc. London. Ser. A. 201 (1950), 417-437.

[Davis 1985] M. W. Davis, "A hyperbolic 4-manifold", Proc. Amer. Math. Soc. 93:2 (1985), 325-328.

[Fricke 1891] R. Fricke, "Ueber eine besondere Classe discontinuirlicker Gruppen reeller linearer Substitutionen", Math. Ann. 38 (1891), 50-81.

[Gibbons 1996] G. W. Gibbons, "Tunnelling with a negative cosmological constant", Nuclear Phys. B 472:3 (1996), 683-708.

[Gromov 1982] M. Gromov, "Volume and bounded cohomology", Publ. Math. Inst. Hautes Études Sci. 56 (1982), 5-99.

[Hantzsche and Wendt 1935] W. Hantzsche and H. Wendt, "Dreidimensionale euklidische Raumformen", Math. Ann. 110 (1935), 593-611.

[Hilden et al. 1992] H. M. Hilden, M. T. Lozano, and J. M. Montesinos-Amilibia, "On the Borromean orbifolds: geometry and arithmetic", pp. 133-167 in Topology '90 (Columbus, OH, 1990), edited by B. Apanasov et al., de Gruyter, Berlin, 1992.

[Hopf 1926] H. Hopf, "Über die curvatura integra Clifford-Kleinscher Raumformen", Nachr. Ges. Wiss. Göttingen Math.-Phys. Kl. (1926), 131-141.

[Milnor 1982] J. Milnor, "Hyperbolic geometry: the first 150 years", Bull. Amer. Math. Soc. (N.S.) 6:1 (1982), 9-24.

[Newman 1972] M. Newman, Integral matrices, Pure and Applied Mathematics 45, Academic Press, New York, 1972.
[Ratcliffe 1994] J. G. Ratcliffe, Foundations of hyperbolic manifolds, Graduate Texts in Math. 149, Springer, New York, 1994.

[Ratcliffe and Tschantz 1998] J. G. Ratcliffe and S. T. Tschantz, "Gravitational instantons of constant curvature", Classical Quantum Gravity 15:9 (1998), 2613-2627.

[Thurston 1979] W. P. Thurston, "The geometry and topology of 3-manifolds", Technical report, Princeton University, 1979. Available at http://www.msri.org/ publications/books/gt3m.

[Thurston 1997] W. P. Thurston, Three-dimensional geometry and topology, vol. 1, Princeton Math. Series 35, Princeton University Press, Princeton, NJ, 1997.

[Vinberg 1967] E. B. Vinberg, "Discrete groups generated by reflections in Lobachevskii spaces", Mat. Sb. (N.S.) 72 (1967), 471-488. Correction in 73 (1967), 303. In Russian; translated in Math. USSR Sbornik 1 (1967), 429-444.

[Wang 1972] H. C. Wang, "Topics on totally discontinuous groups", pp. 459-487 in Symmetric spaces (St. Louis, MO, 1969-1970), edited by W. M. Boothby and G. L. Weiss, Pure and Appl. Math. 8, Marcel Dekker, New York, 1972.

[Wielenberg 1978] N. Wielenberg, "The structure of certain subgroups of the Picard group", Math. Proc. Cambridge Philos. Soc. 84:3 (1978), 427-436.

[Zimmermann 1990] B. Zimmermann, "On the Hantzsche-Wendt manifold", Monatshefte Math. 110:3-4 (1990), 321-327.

John G. Ratcliffe, Department of Mathematics, Vanderbilt University, Nashville, Tennessee 37240 (John.G.Ratcliffe@Vanderbilt.edu)

Steven T. Tschantz, Department of Mathematics, Vanderbilt University, Nashville, Tennessee 37240 (Steven.T.Tschantz@Vanderbilt.edu)

Received March 4, 1999; accepted March 9, 1999 